\title{
DRAFT
}

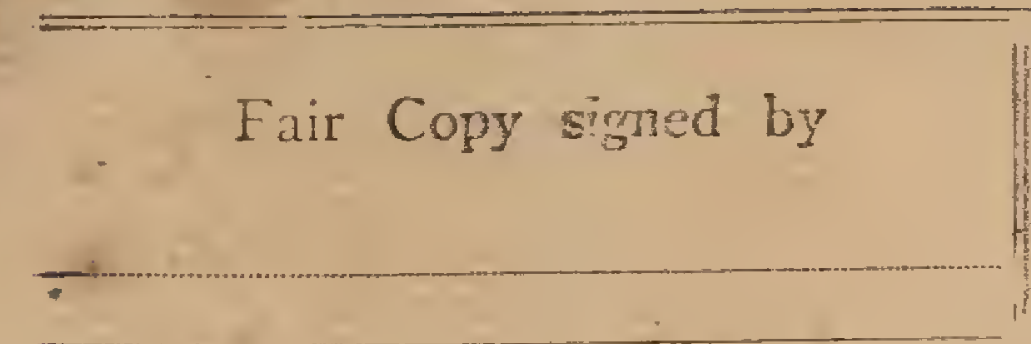

$\left.2 x^{7}+8\right)$

To

Sir,

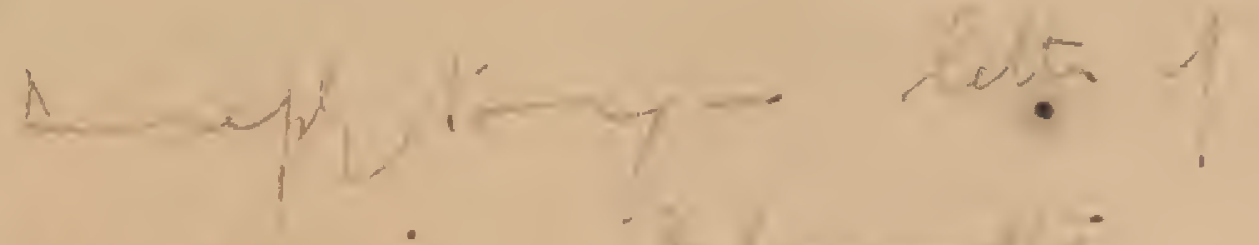

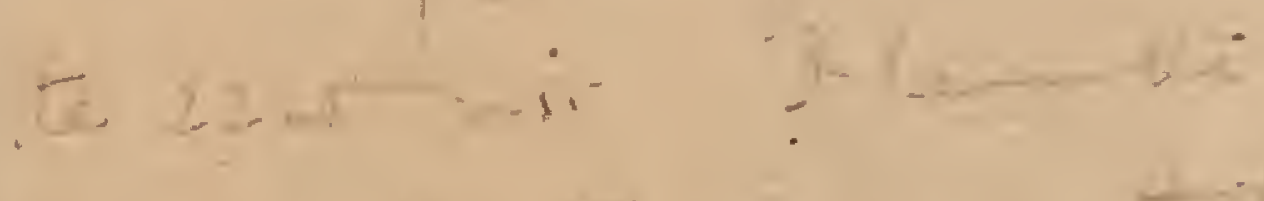

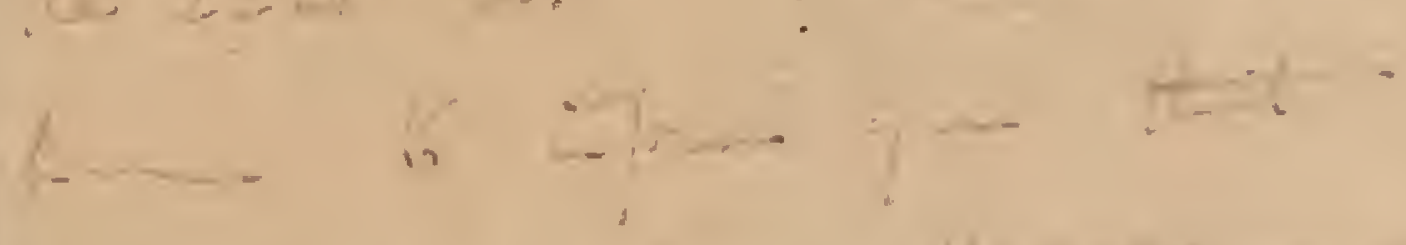

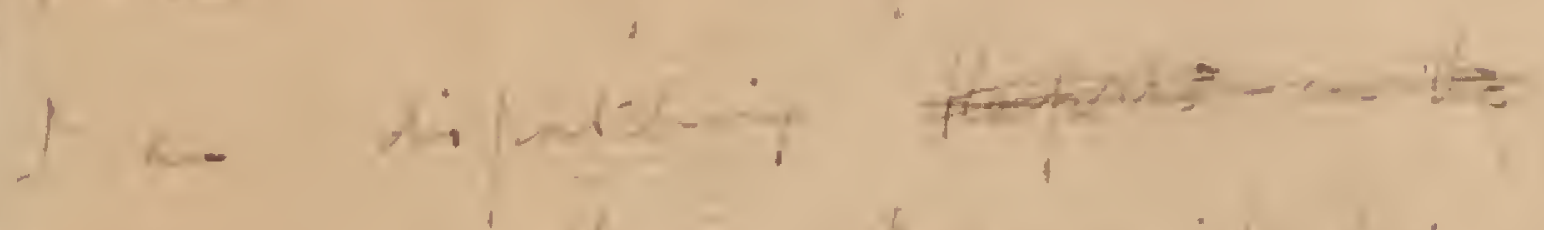

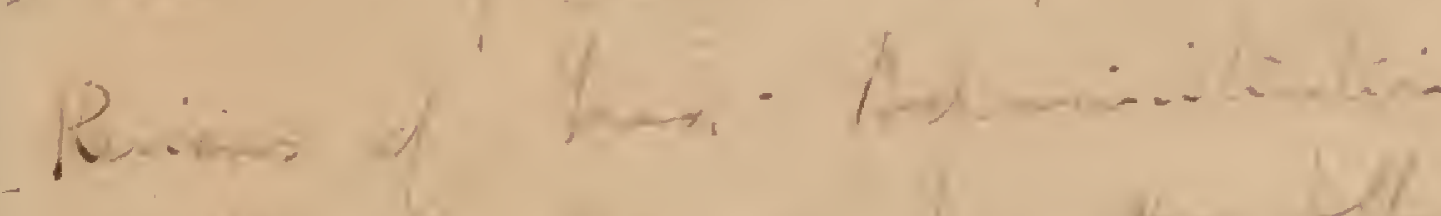

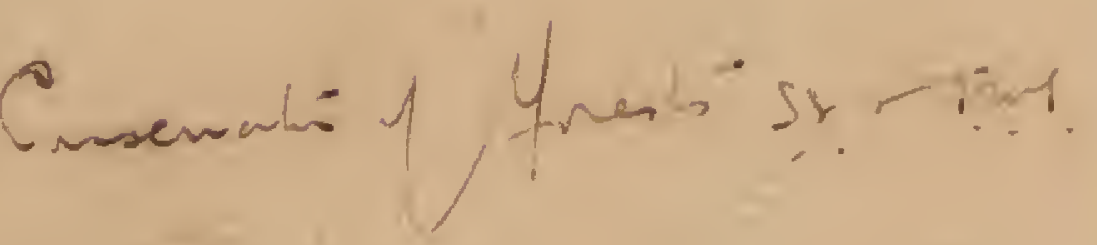

$1,1$.

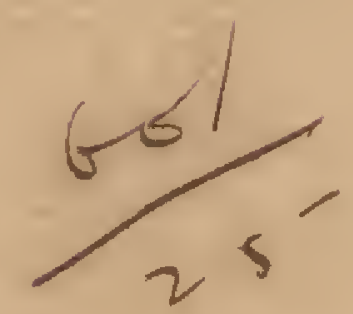

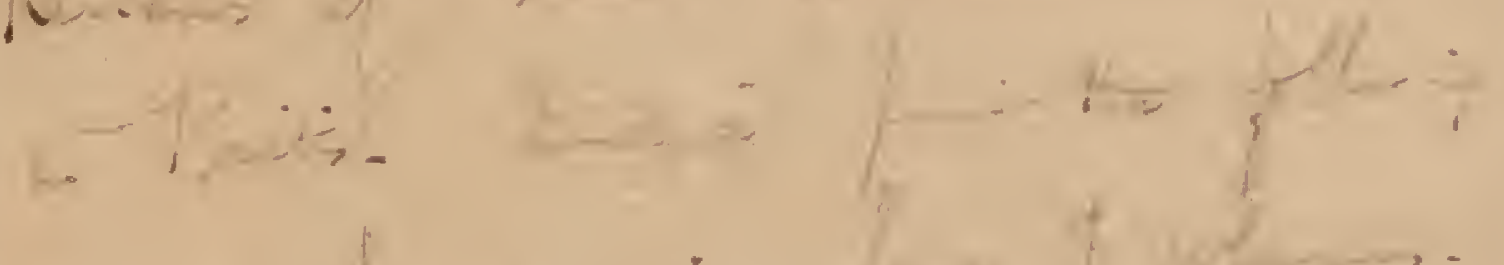

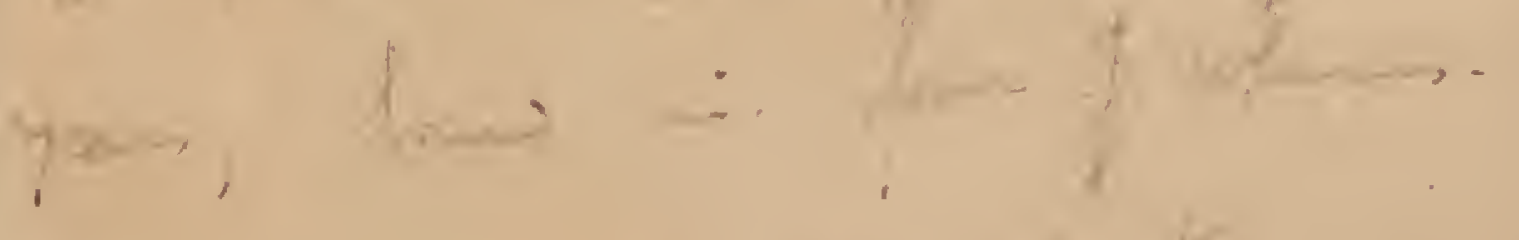

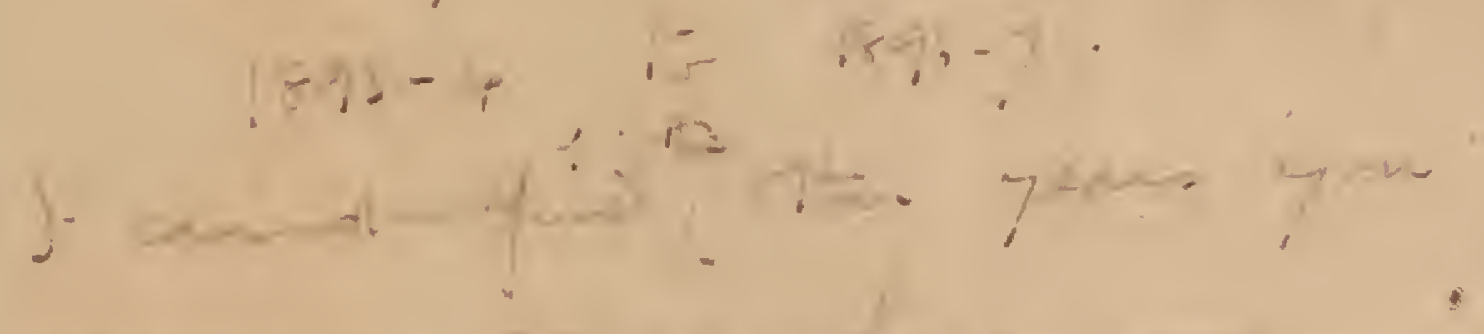

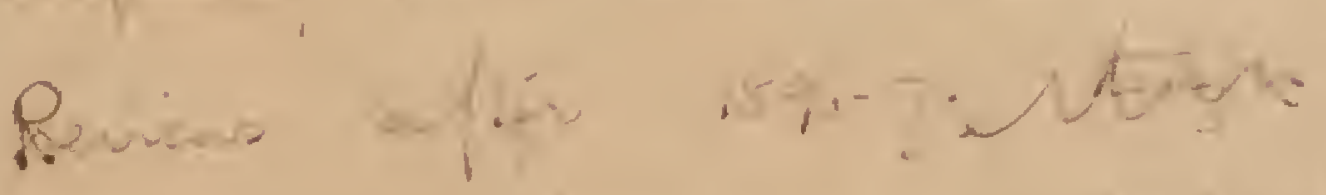

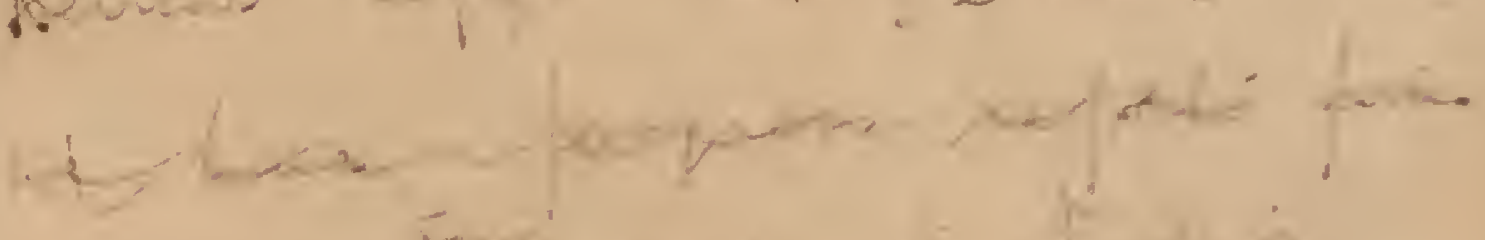

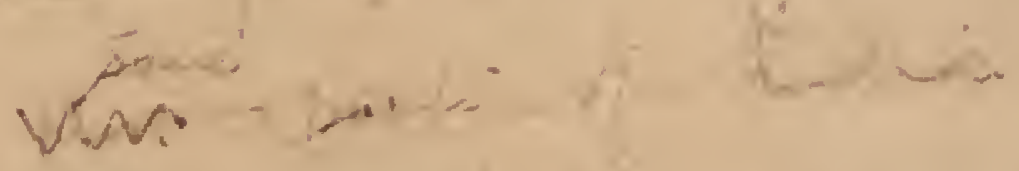

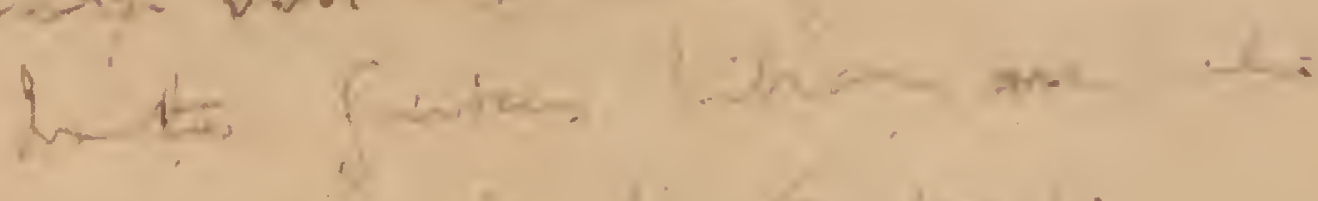

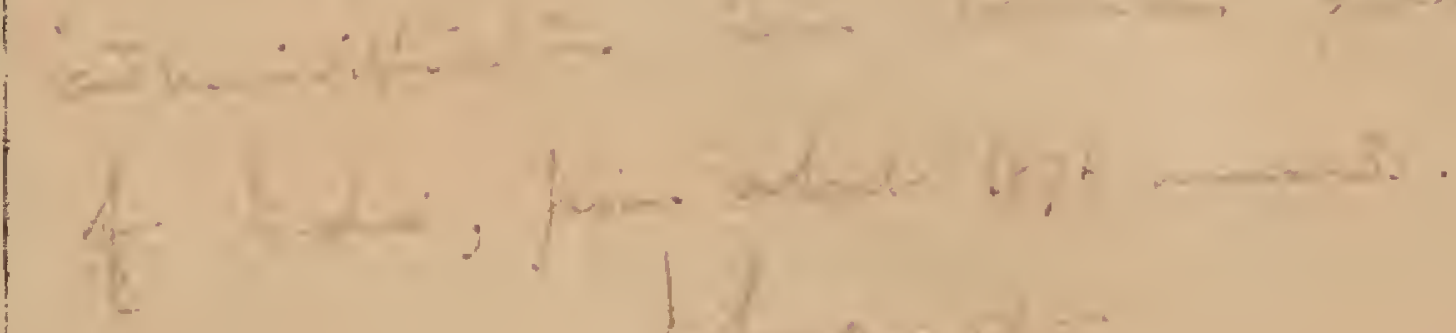


It is requested that the following number be quoted in the reply to this letter. No.
OFFICE OF CONSERVATOR OF FORESTS

F. M. S. \& S. S.

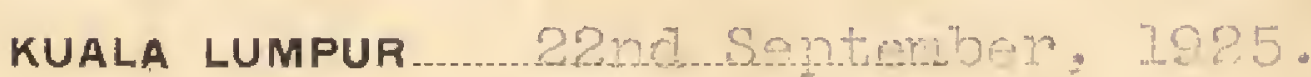

gir,

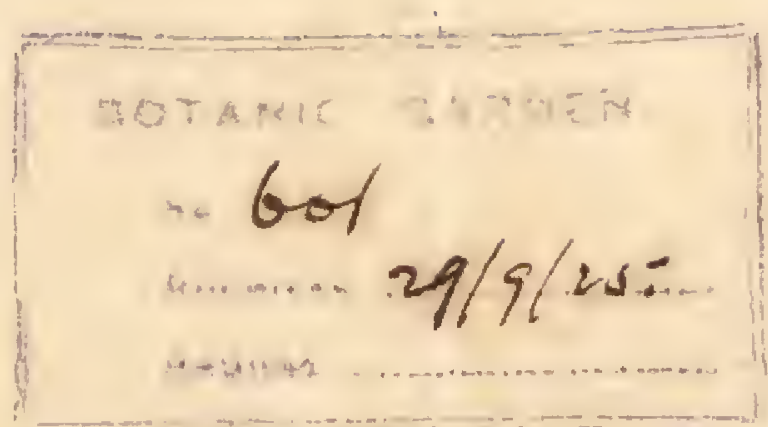

I heve the honom to inform you thet I am sorious

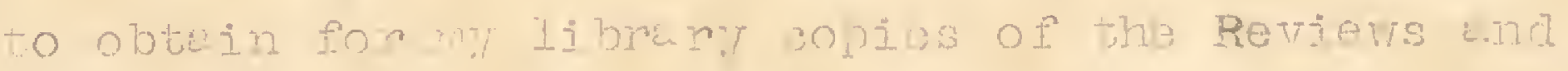

Statigtics of Forset Adringtudton in Indifk. Copios

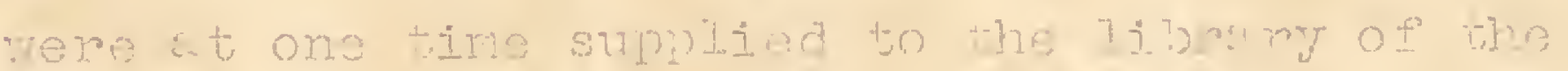

Botanical Gordens, Singpore, but I ins no ne sur of no

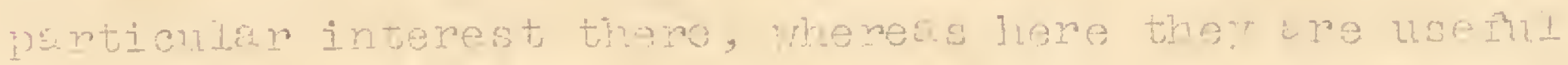

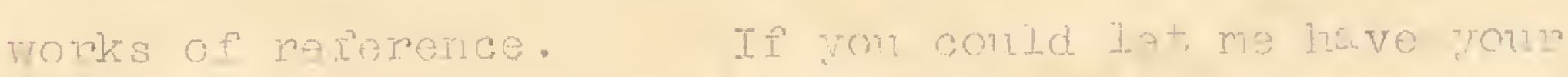

copies, I sinomid be aytrame

The missins volumes in w ofice a wer for the

tolioning verns:-

$$
\begin{aligned}
& \text { Prion to } 1876-77 \\
& 1.877-78 \\
& 76 \cdot 7-81 \cdot 2(5 \times 6.778) \\
& 77-8 \\
& 42-3-85-6 \\
& 1882-83 \text { to } 1894-95 \\
& 9+4-9+5-9 b-7 \\
& 1896-97 \\
& 1899-1900 \text { to } 1904-05 \\
& 1907-08 .
\end{aligned}
$$

To,

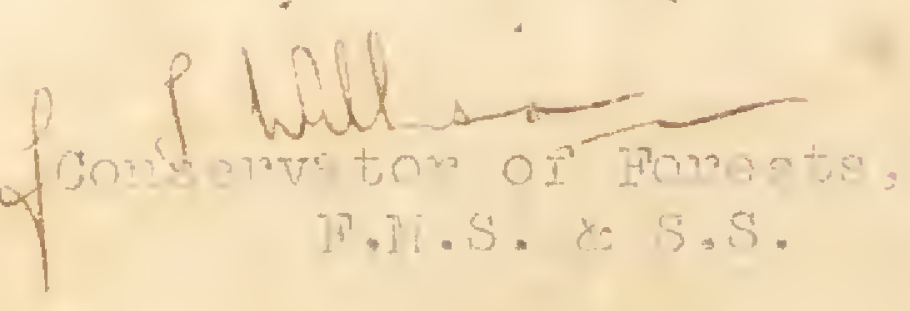


63 in C.F. I/25.

(Gen. 67)

\section{Official Memorandum.}

2and October, 1925.

From cons: of Forests, To Director, Botanical Gardens,

F.M.S. \& S.S. Singapore.

\section{Your 2 in 612-25.}

Amongst the books you have kindly sent me are two copies of Kurz's Preliminary Report on the Forests of Pegu. As this book is of botanical as well as forest interest, I think perhaps it may have been sent by mistake, so I therefore returnone copy.

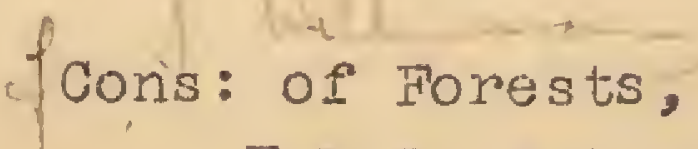

$$
\text { F.M.S. \& S.S. }
$$


(e)

DRAFT

(G 17)
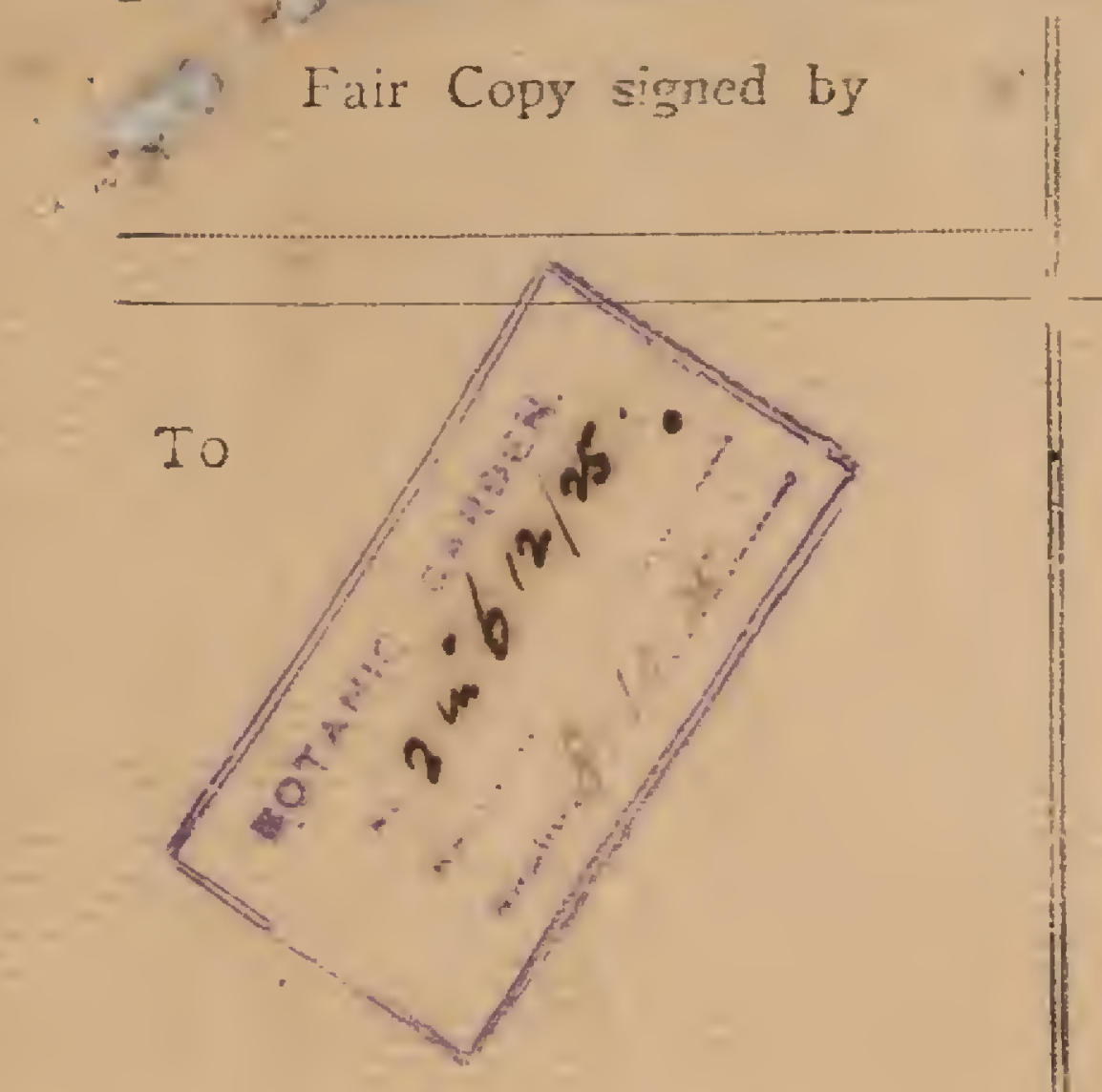

Sin,

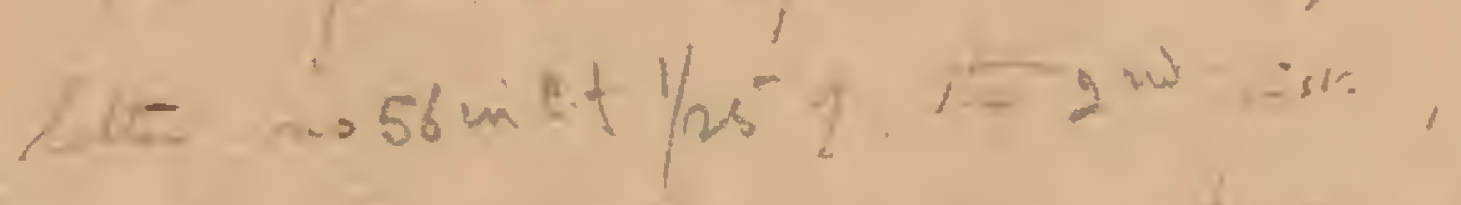

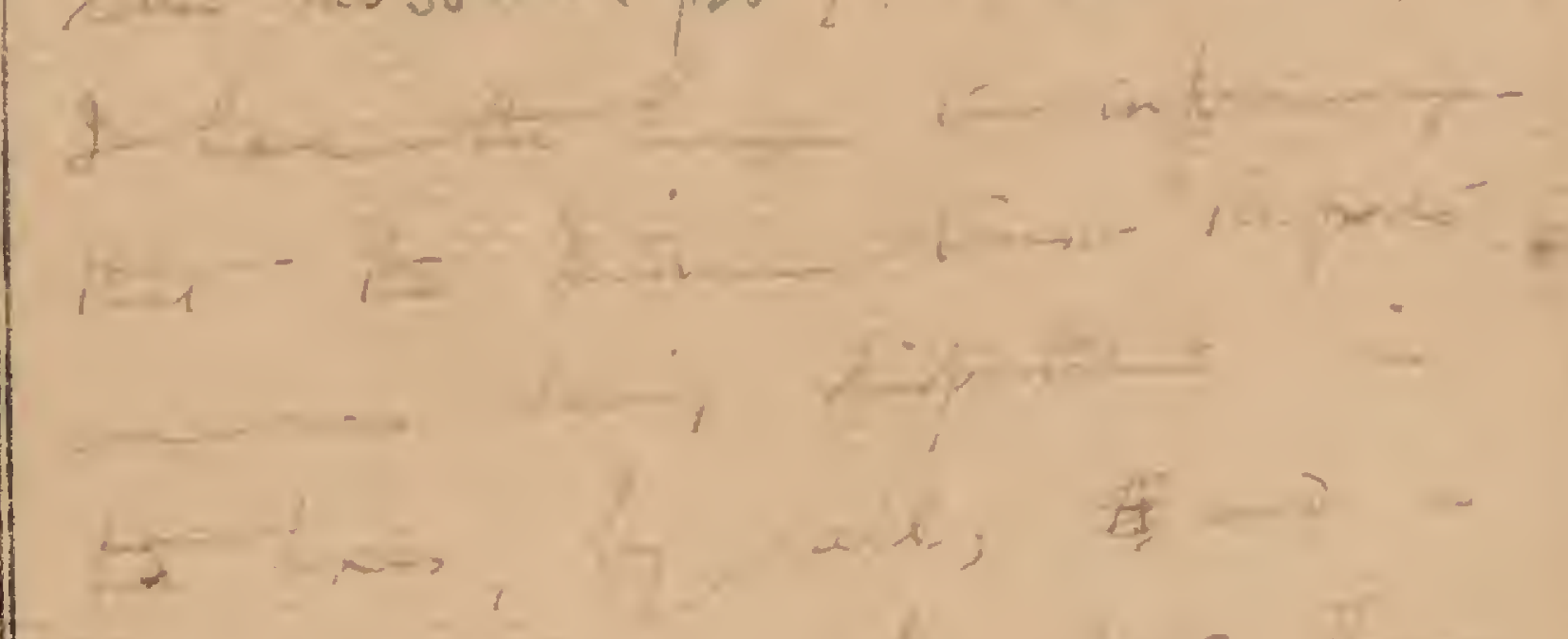

Cineruation fras

$f=x+3$

$k+k+2=0$

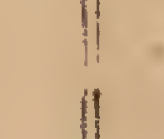

81.4514 
(c)

DRAFT

Fair Copy signed by

$$
6 / 1+y-
$$

To

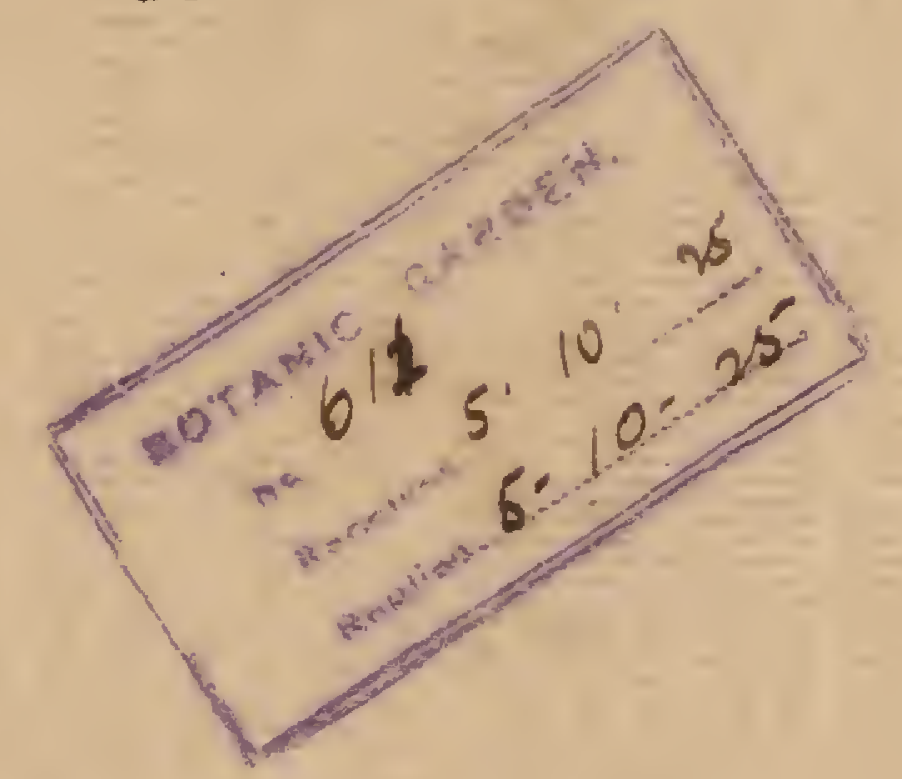

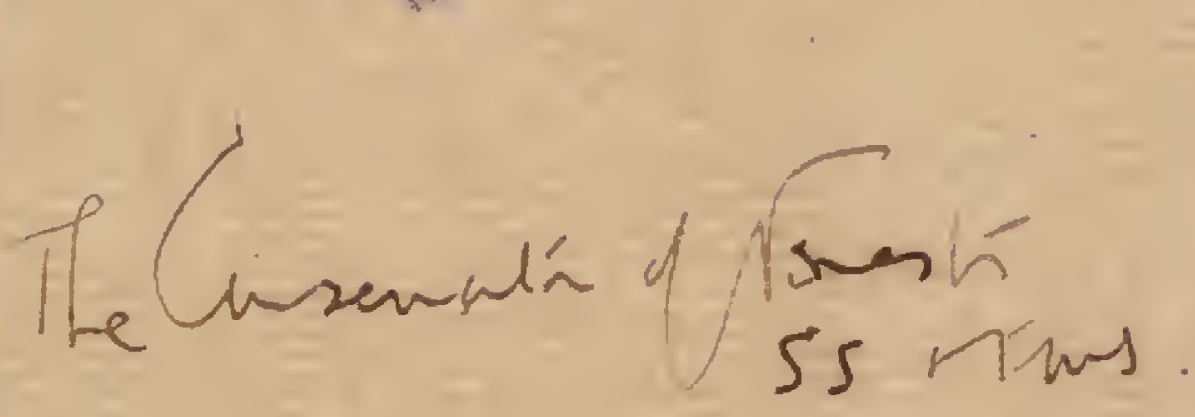
imala L-im
$\operatorname{Sin}_{\mathrm{p}}$

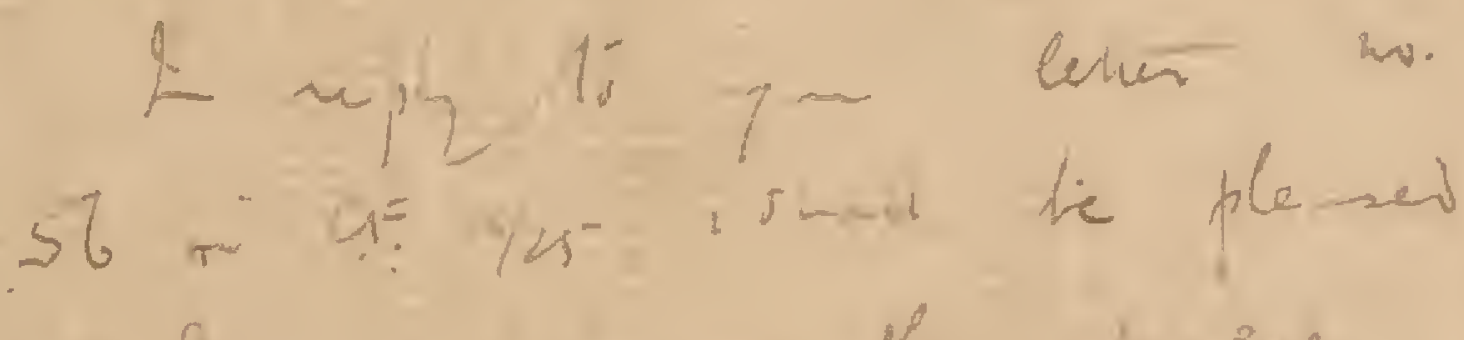

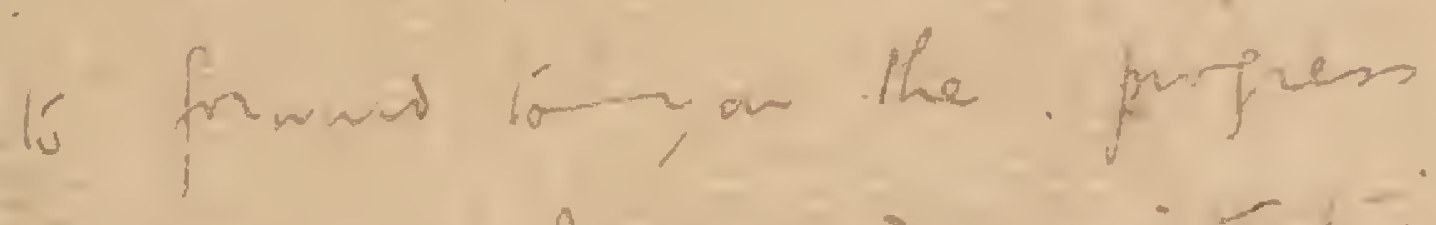

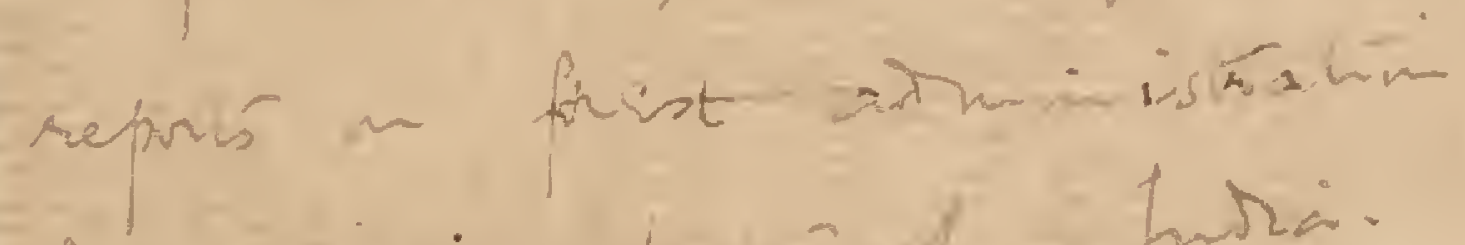

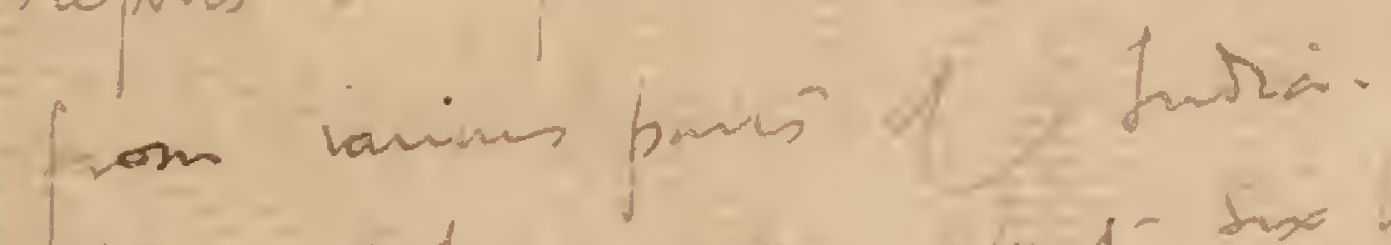

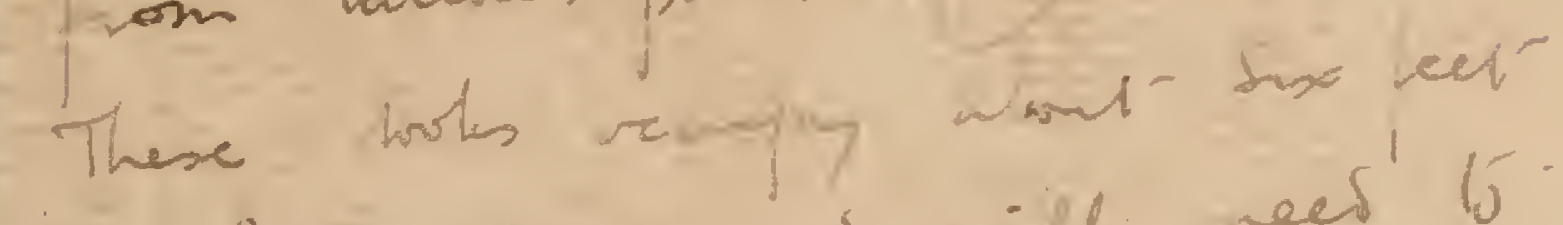
of Dell swa and ill mees 15 ve saded $\therefore$ hoxes. Iniel wifl y- wiven the we sedy ingatpricic.

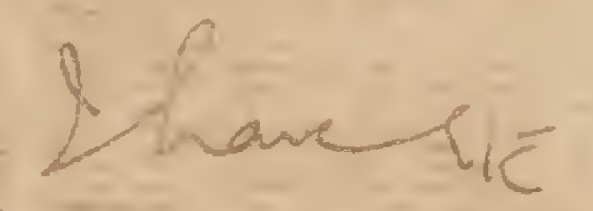

Er. 
It is requested that the

following number be quoted.

in the reply to this letter.

\author{
OFFICE OF CONSERVATOR OF FORESTS \\ F. M. S. \& S. S.
}

No. 56 in C. F. $1 / 25$.

KUALA LUMPUR 2nd octobera, 1925.

Sir,

I have the honour to acknowledge with many

thanks the reviews of Forest Administration in

India for 1876 - 1877 to 1885 - 1886,

1893 - 1894 to $1896-1897$.

We should also like to have the progress reports

of forest administration from various parts of

India, if you can spare them.

I have the honour to be,

Sir,

Your obedient servant,

To,

the Botanical

The Acting Director of/cardens, 
hu Conservaton of Dorests, s.s, dF M. S. Thala Tusufun

Iist of Progress Reports on Forest Adninisration Prom various C. Parts of India.

Forest Administration in Coorg for the year $1876-1883$

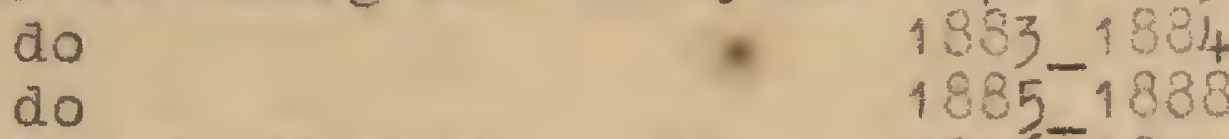

3.

4.

5.

B.

Forest and other vegetation of Pegu do

Forest Administration of Bengal

do in Andamans do

do

do in Burma

do

do ảo

do

do

do

do

do

do

\section{of Assam}

do

do

The Forests in Kulu

do

The Forest Administration in B. India do

Progress report of the Forest Survey, India do

Reports on Indian Forests

Forest Adm. Reports of Several Provinces

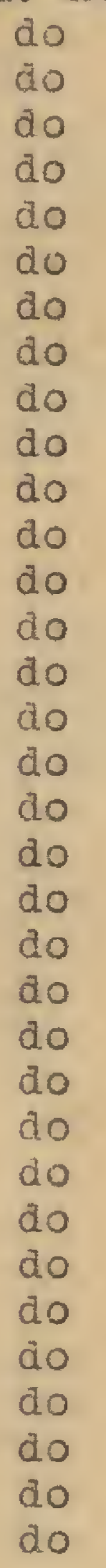

Central Provinces do

N.W. Prov, \& Oudh

do

do

do

do

do

Hyderabad

Districts

34 .

35 .

36.

do
do
do jab
do

\section{Ajmere}

Ajnere \& Mawara

Madras

do

do

do

do

do

do

do

do

do

do

do

do

do

do

do

do

do

$1883-1804_{+}$

$1876-1877$

1836-1837

$1885-1888$

$1876-1=33$

$1883-188 \times 8$

$1888-1889$

1875

1875 Reed. 23-x-25.

1876-1883

$1883-1384$

$1885-1386$

$1876-1283$

$1883-1884$

$1885-1888$

1887

$1883-1884$

$1.884-1886$

$1882-1883$

$1334-1337$

$1877-1373$

$1874-1875$

$1876-1833$

$1385-1383$

$1876-1882$

1881

$1883-1884$

$1885-1886$

$1887-1838$

$1808-1889$

$1876-1883$

$1883-1884$

$1885-133$

$1876-1883$

$1333-1384$

$1885-1888$

$1876-128 ?$

$1879-1288$

$1881-1883$

1383

$1883-1884$

$1884-1887$

$1887-1883$

$1387-1888$

$1389-189 ?$

$1892-1895$

$1895-1838$

$1808-1901$

1901-1904

1904-1907

1907-1908

$1908-1910$

$1910-1913$

$1913-1916$

1916-1919

1919-1921.

Tomanded $f / x / 23$ 
Hear suntecu.

- Can un suen lel mo

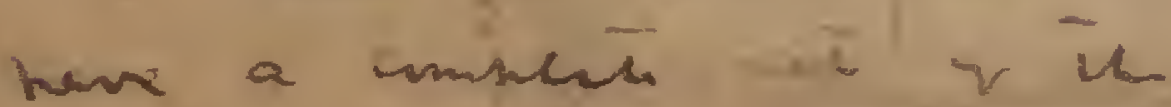
anmial wester on th $2=$.

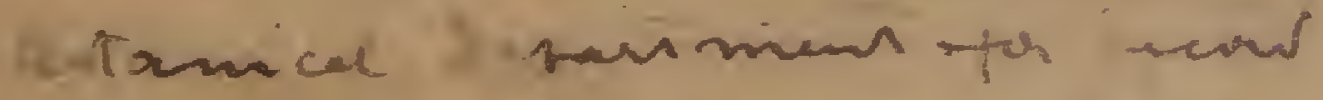

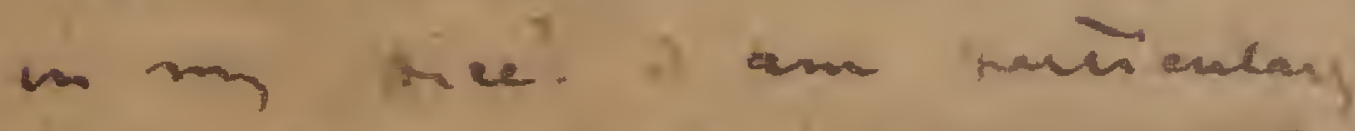

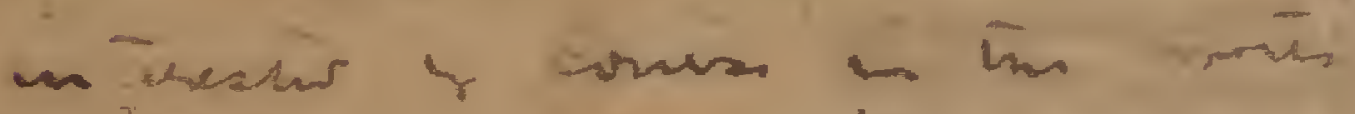

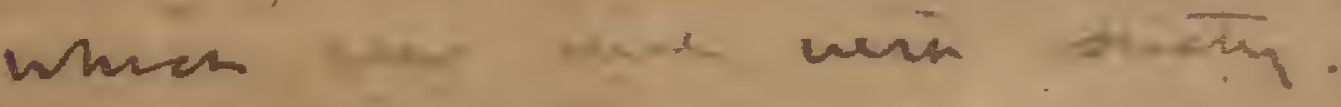

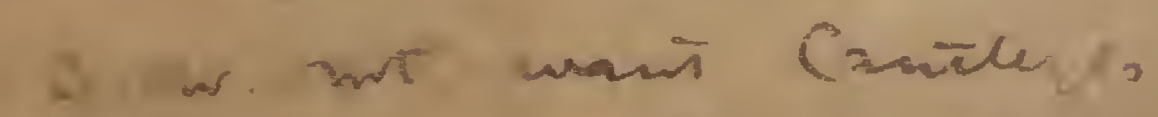

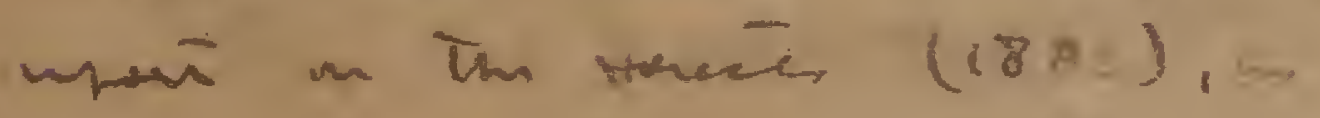
2 sour 2 on 3 epries y inat.

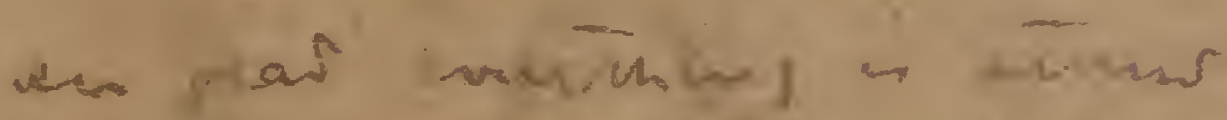
coms. "TE hon' Fondex.

Fi ush 

ADentorandm.

(G 30)

From

Bntanie farien

To

Sinjapre

Dran Bunill,

tevenitio lettan is follows:

(1) fin hinclim ahut Riskis Ftria

(2) Inm Aisurologiir at tiusa

(3) form incleland veletry is the papen about 7 ivels inclases locaily sent on tis yn yestersay.

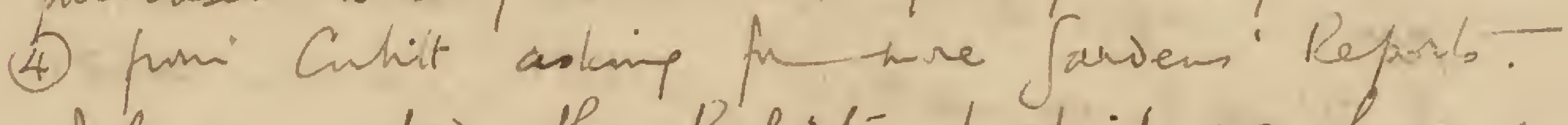
thave markes the Repurts I phich we have no cuphicates. for the years hepo 1886 /michonin $1875+7876)$ Inviajo has duplicales which were formerts lmos ip lógether. for 1893, 1897, 1899 (Culittspara 2)

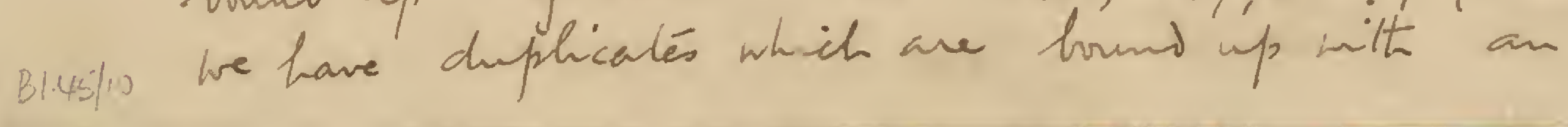


incomplete set, o sen them This would have is the

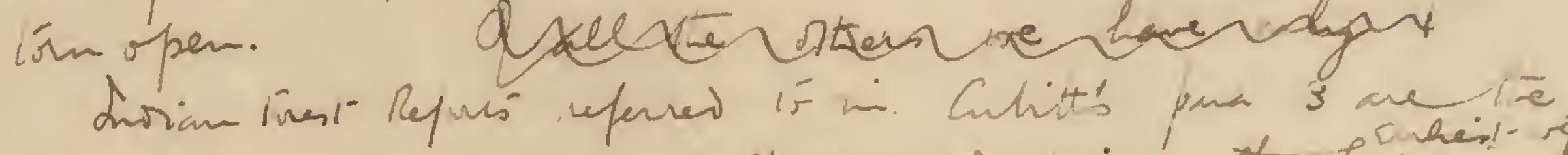

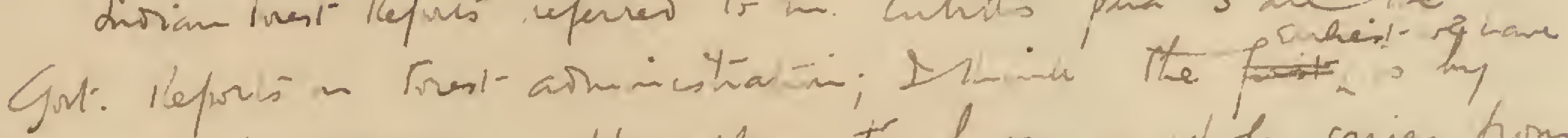
Brants in 1879-8. We apprized to have a while series from Then currant, I don's know how complete.

Cults's para 4. (a) the fred report referres.75 appears s be Camileyis 8 1883 - there is wo ot ion about this time

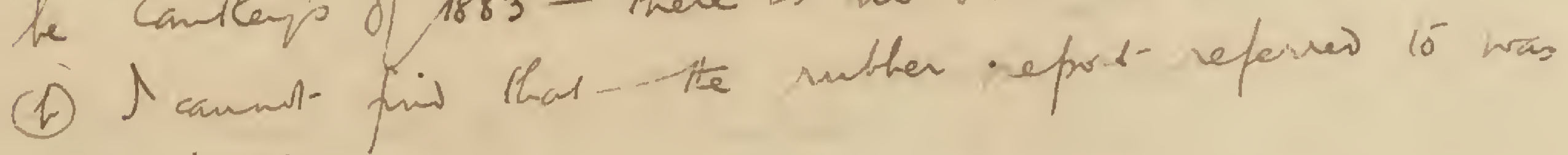
Ever sued.

paar 5- refer to various Colonic frest-repouts. I will send on anything. $7^{\mathrm{m}}$ thin kkk we cam sens. In o Buntérysy guides have arrives.

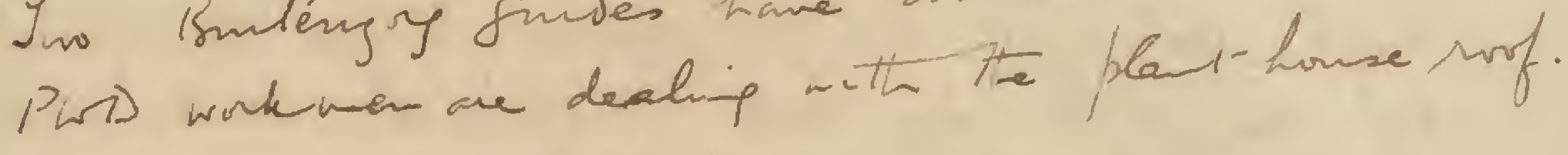




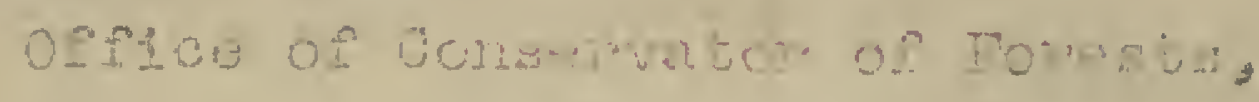

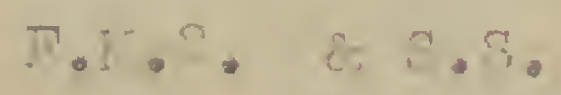

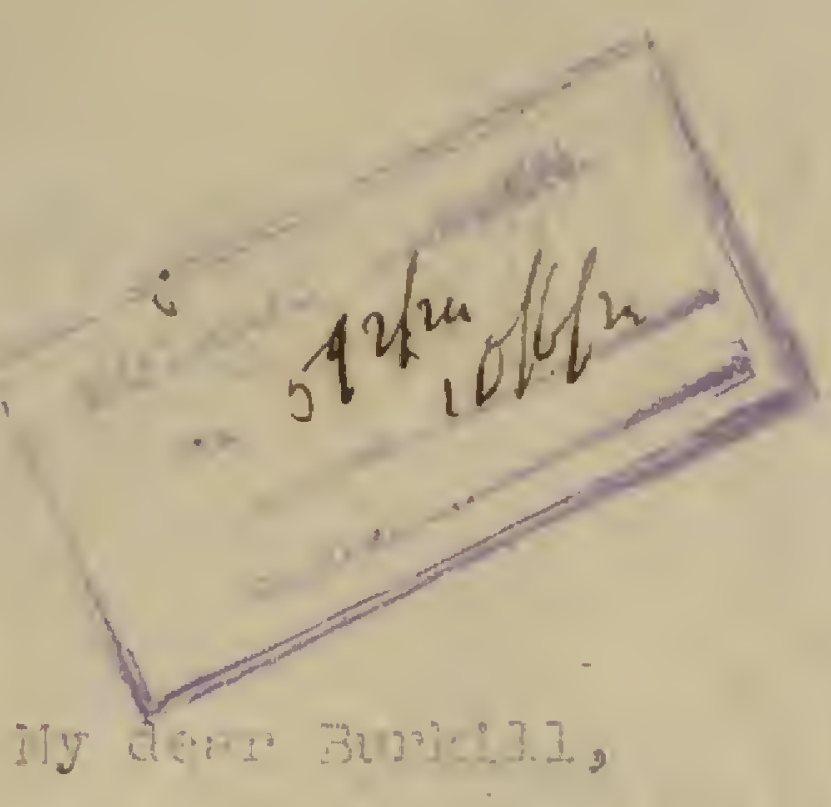

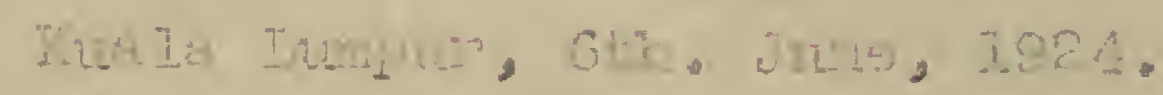

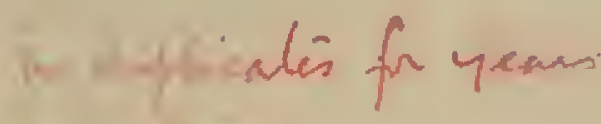

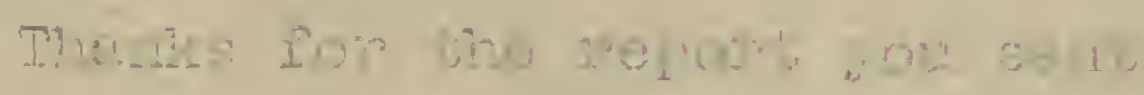

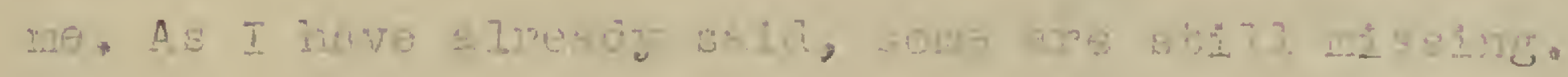

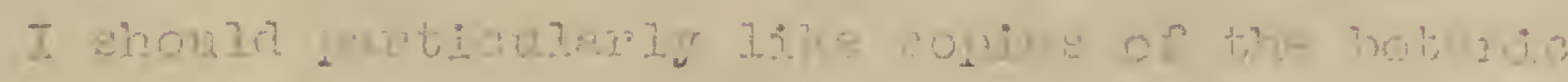

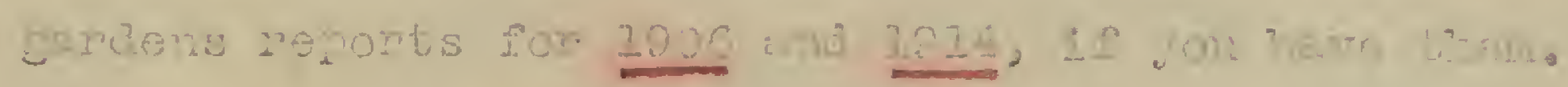

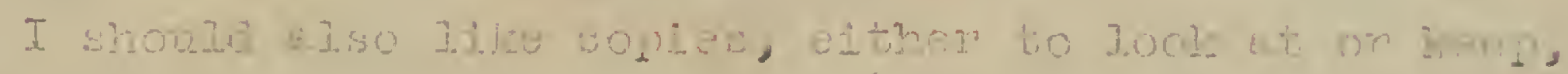

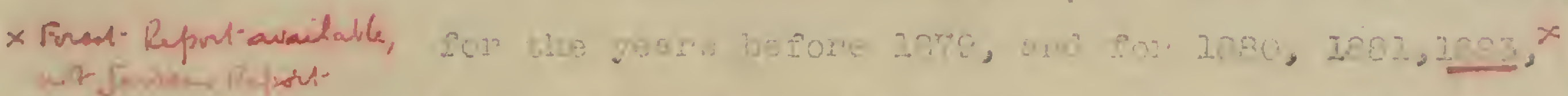

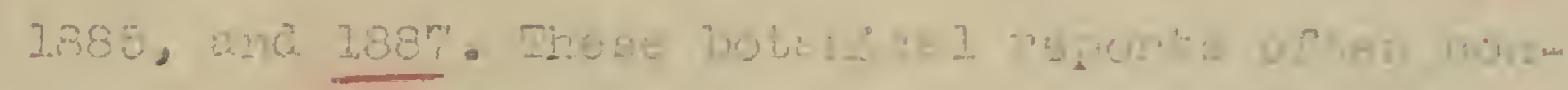

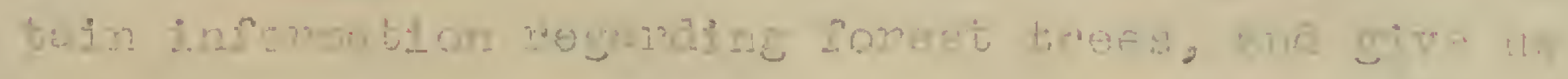

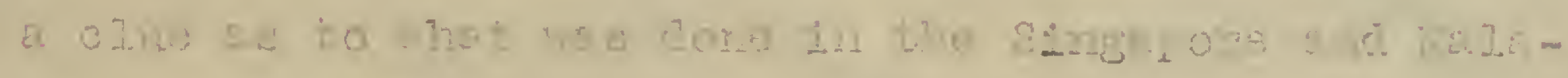

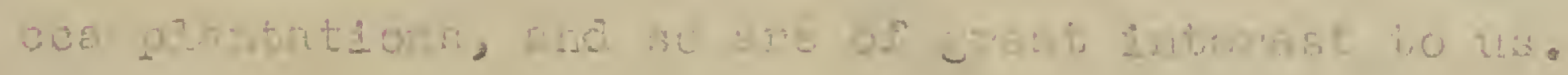

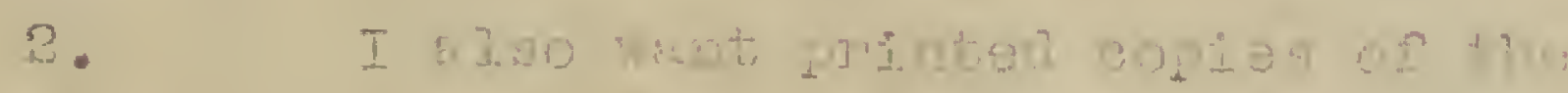

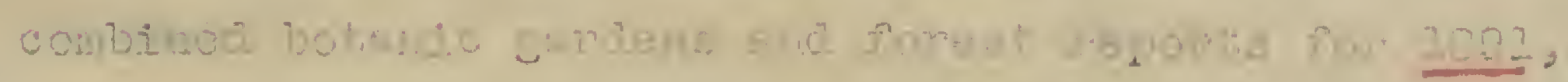

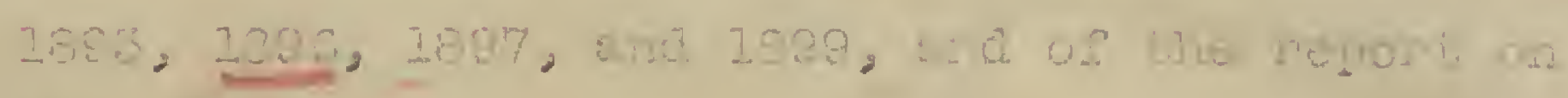
forvester for 7906 .

\section{At the button af gage of of the}

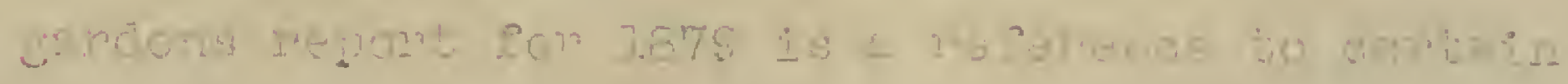

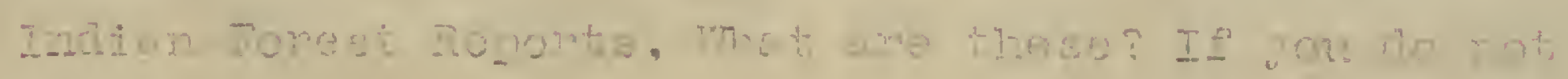

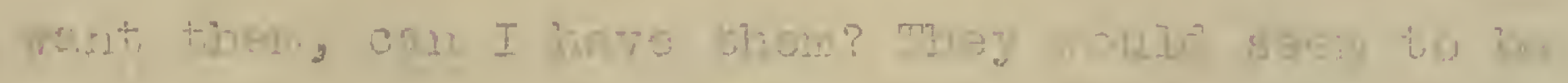

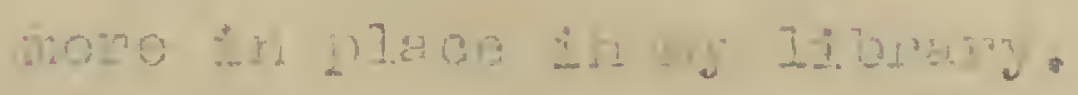

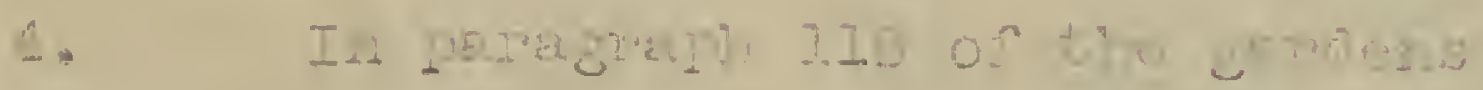

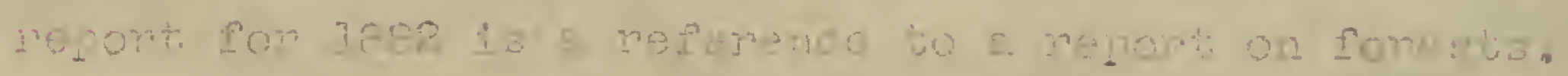

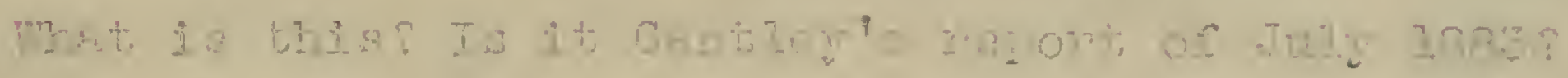

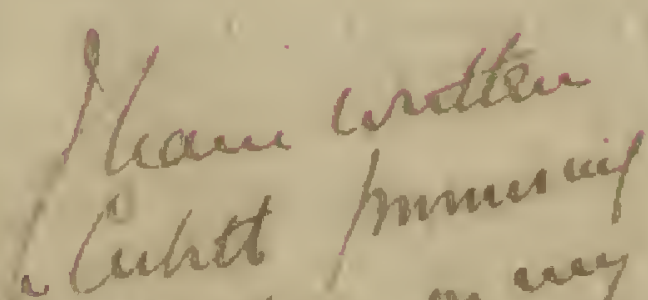

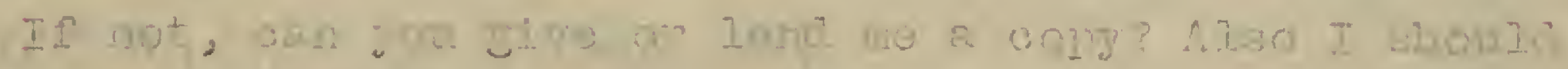
alliution mary

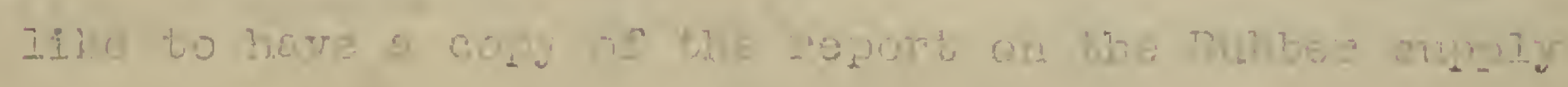

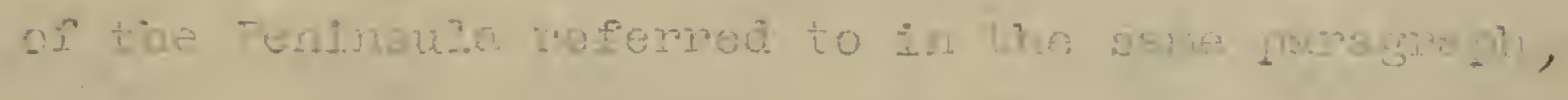
Whamer hes B1 45 
8.

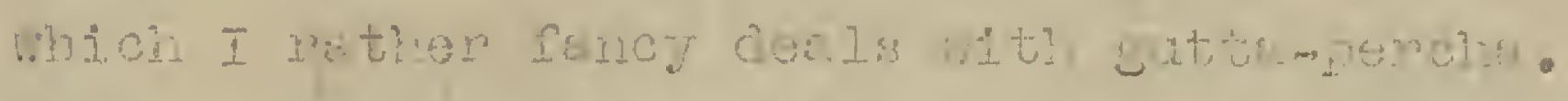

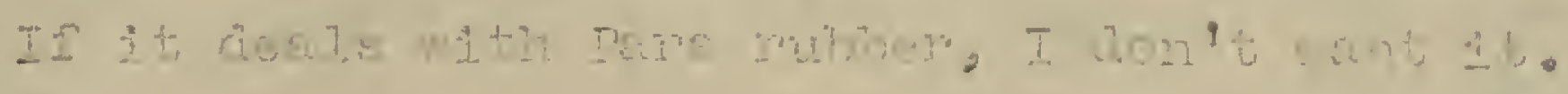

5. At the botion of pege 5 of the famens

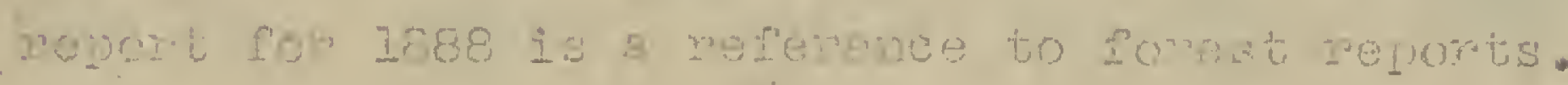

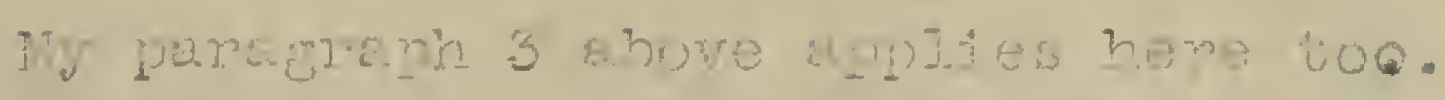

6. If you have any boures on cropotivy

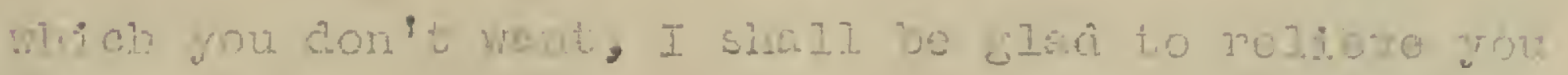

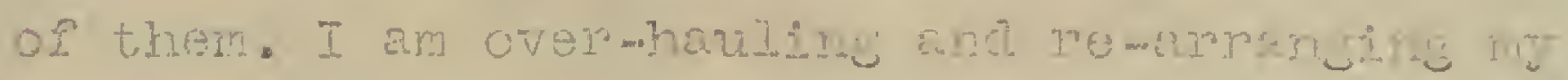

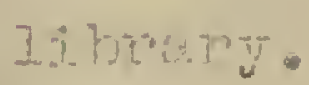

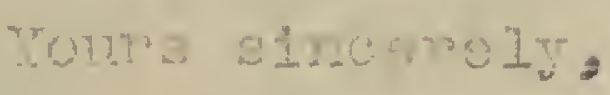

Clain

B1 45112

$I S T / Q P$ : 


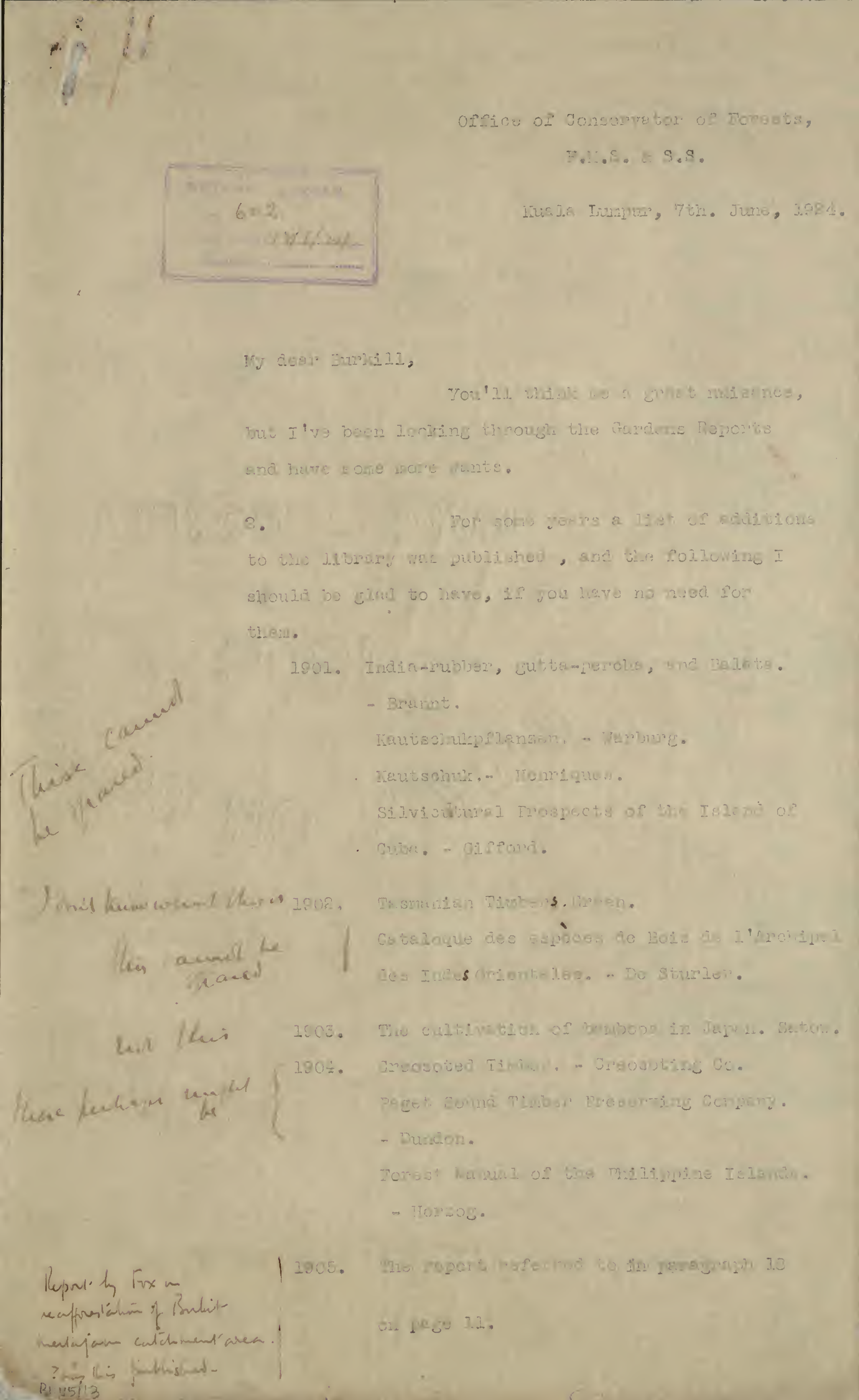




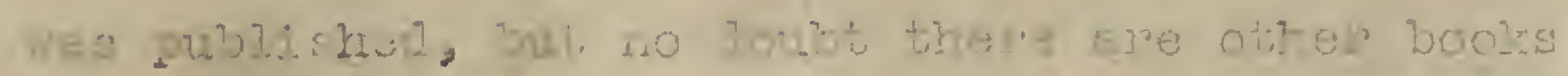

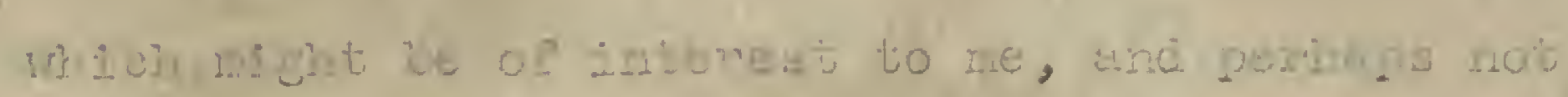

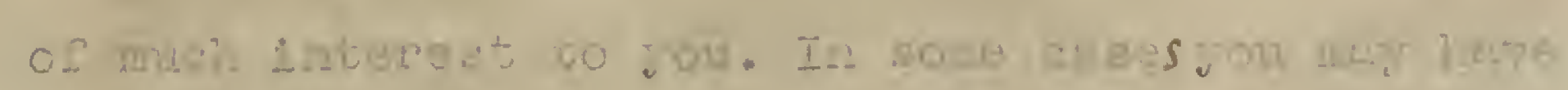

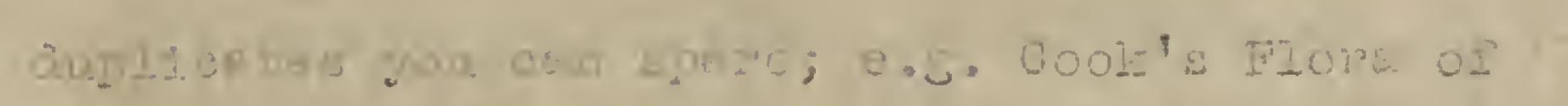
Sombsy.

Tours giaecinely,

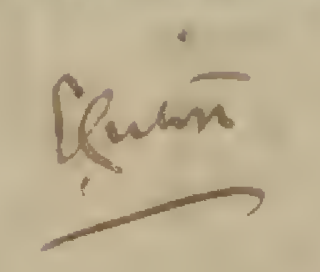

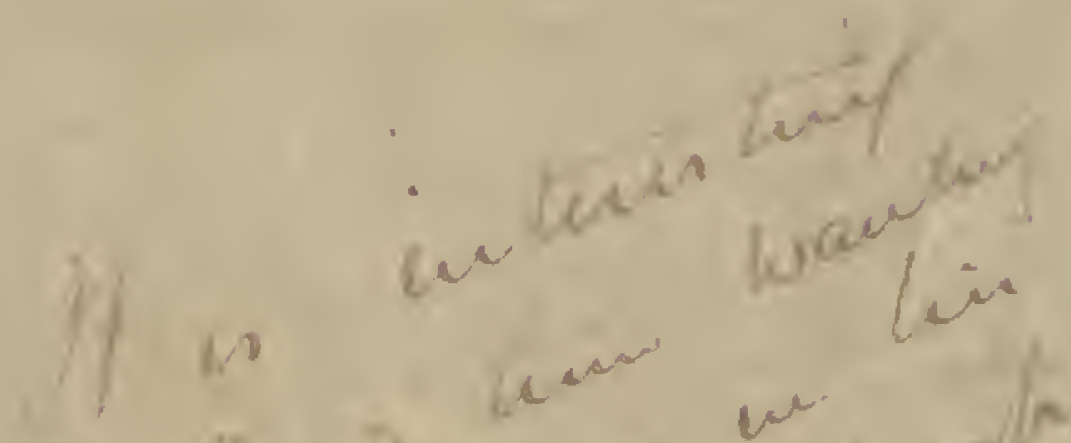

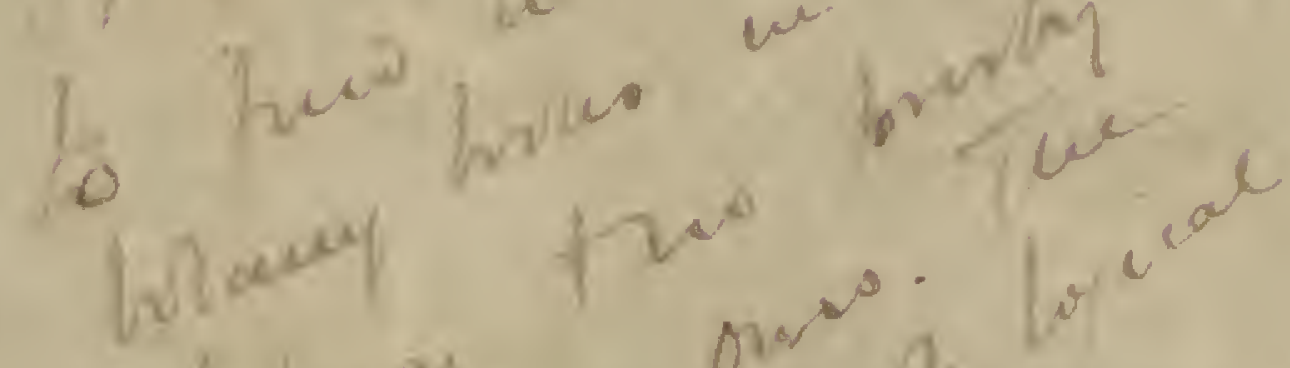

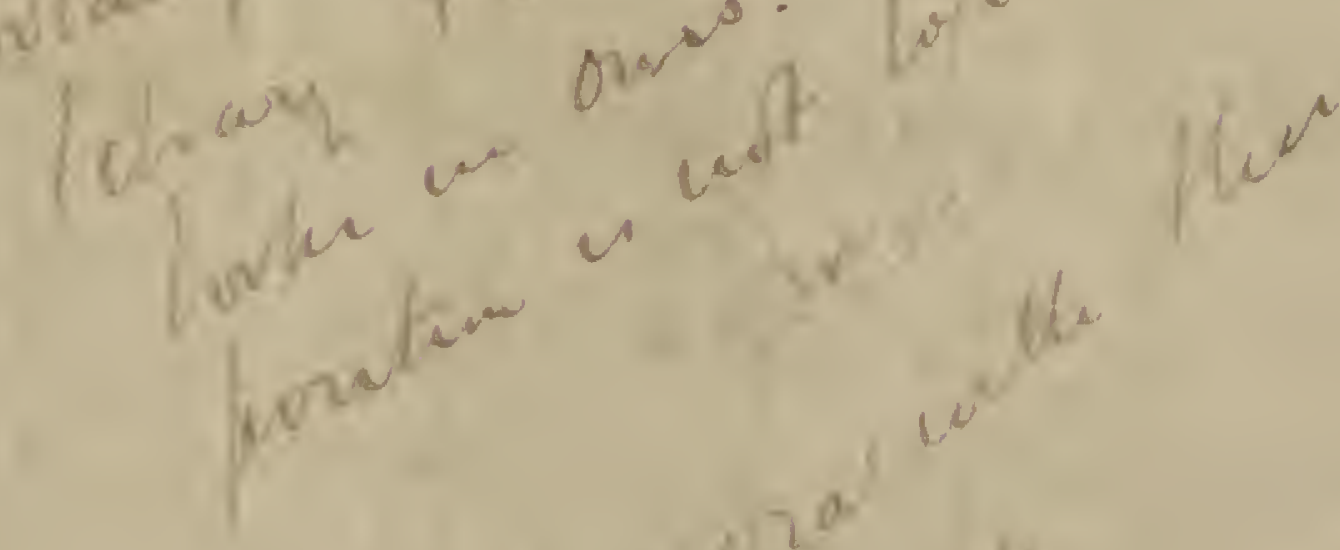

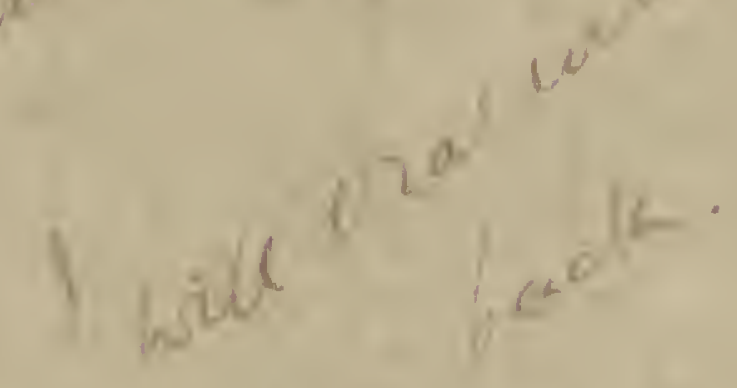

$$
\begin{aligned}
& \text { son }
\end{aligned}
$$


From Whom

Place

Date

SUBJECT.

Qubaris

- Consenvalir y hrora arks for broks.

Comcoted Papers. MINUTES.

Bis nol rearmatla to oay that a

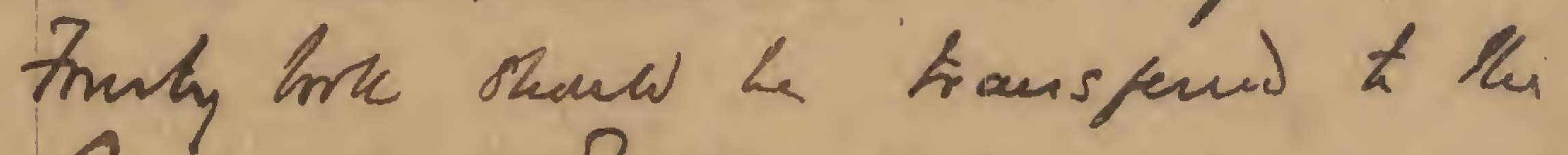

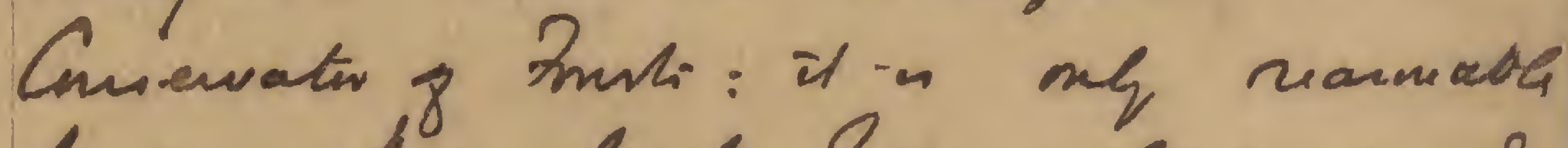
\& say that prosty hrases which we of notine oluow te tranfenit.

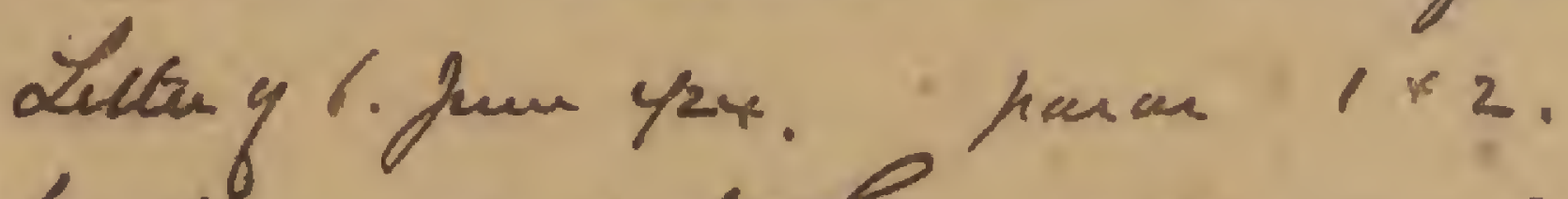

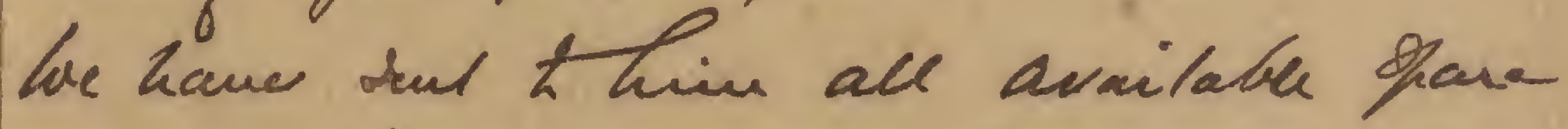

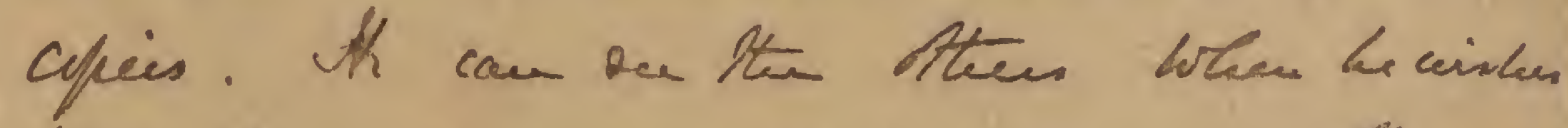

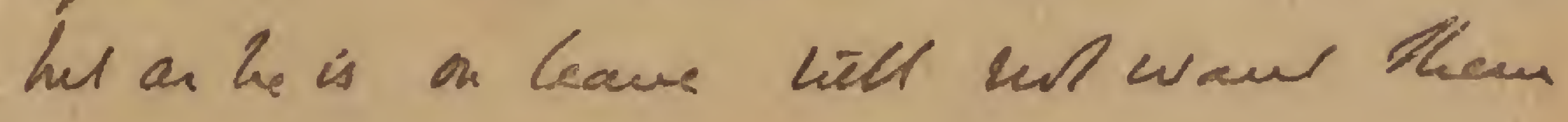
kow.

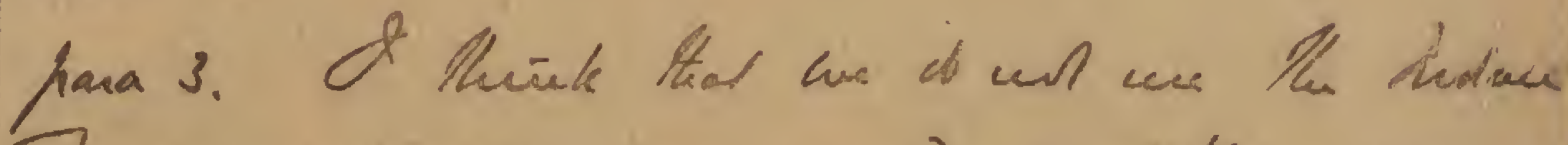

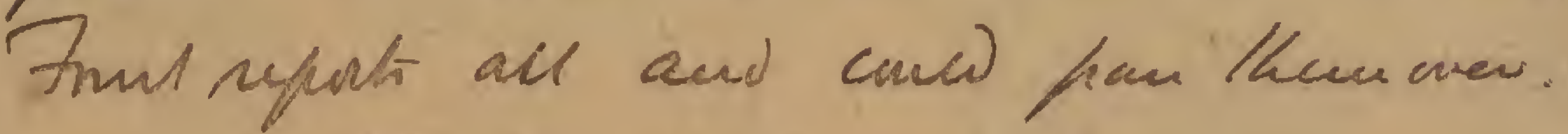

trano 4. To wher the ke ryfen?

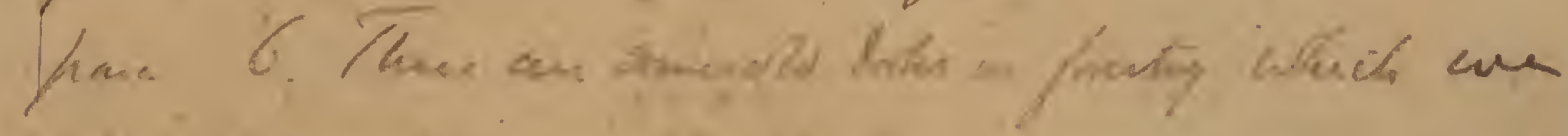

$B, 45 / 15$

$1.45 / 15$ 
Lellon 7 foum y24 frow 2.

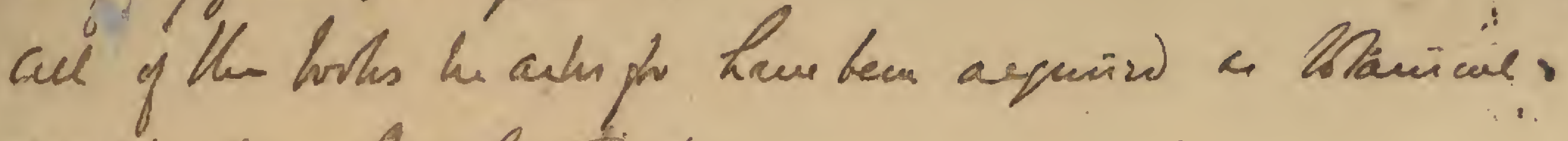
excepl. You Cussdes tivections

and wen Znis nefued funthined.

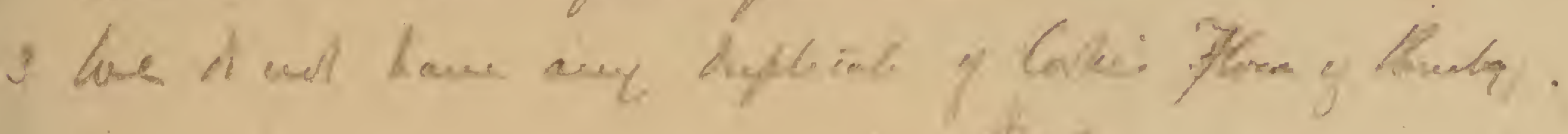


No Minutes should be written on this page. A separate half $=$ sheet to be used if required.

$\therefore \quad$ i

B1 $45 / 16$ 

Torence, R. Mupes Urà Romen

le o\& Octobru 1902

Now cher X-Riedley.

I. ne donte pot quer ur rain vout abra enobye ene copie de now - encentueretion det equicy de Carbiny et Lacuronoiops, qui is éth que un prolpeetuy de ma monographier on toutes iitépies serout reprobing en phototypie de groubu nutarebu wichouthlows a'therbur Lo veguctes de 
Le coufidere Anigapounty

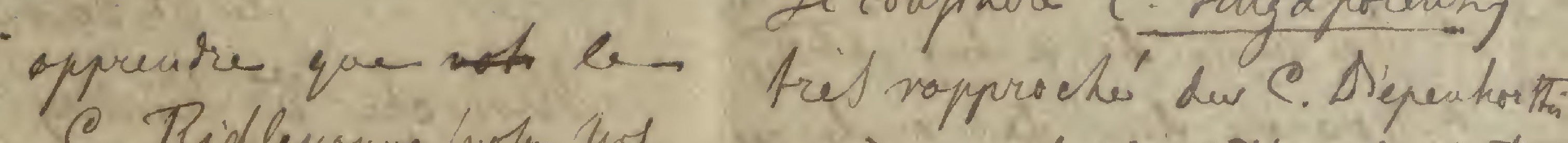

C. Ridleyony voto ust.

6961 et 90501 me prodict

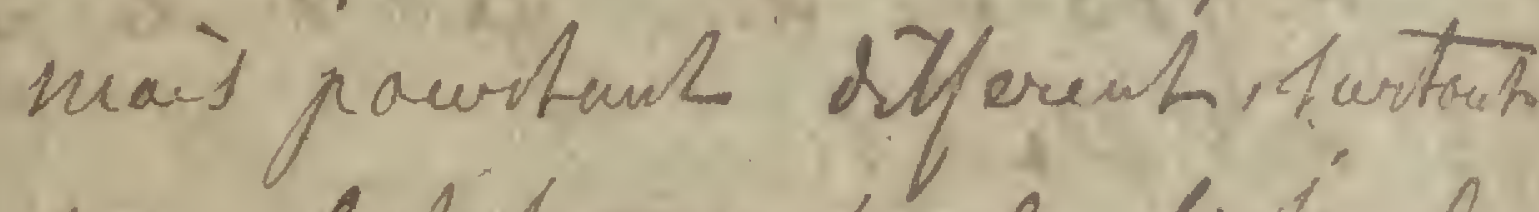
pour let tegments be lisfecules fot de friuts de cetre ifpo Laut prinily our poilts tur

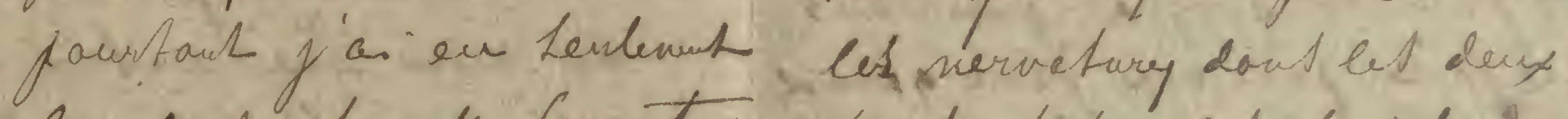

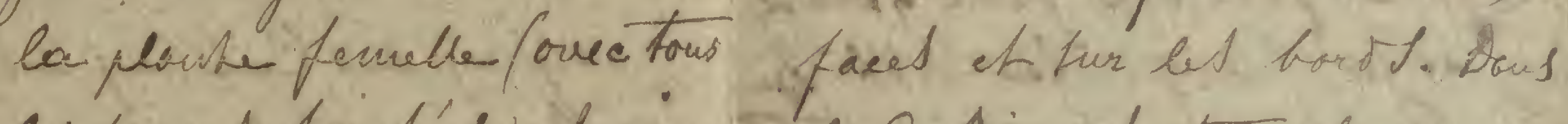
es feurt toubés et je de. Diejentorttis la nerreme

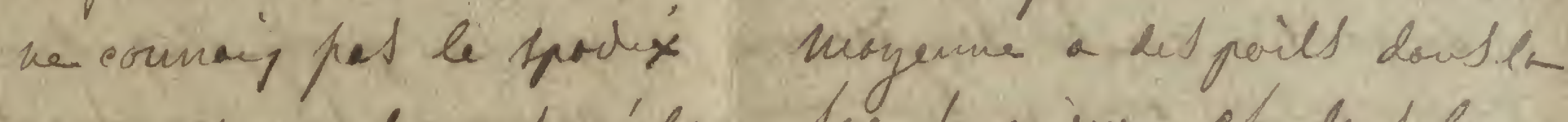

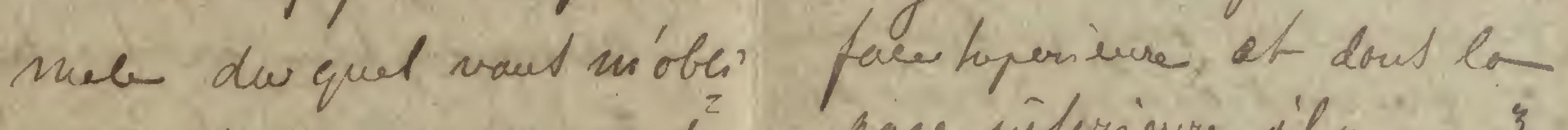
qerg beaneoup ti vous page niferience ifly a s powny un enoryer un fro neviruef changes da pails. Precen le yooby a du C. die

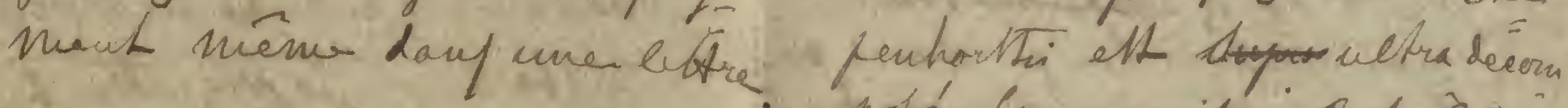
poké (ou ramifie 2 foiss) 
Aacers quil els himplements

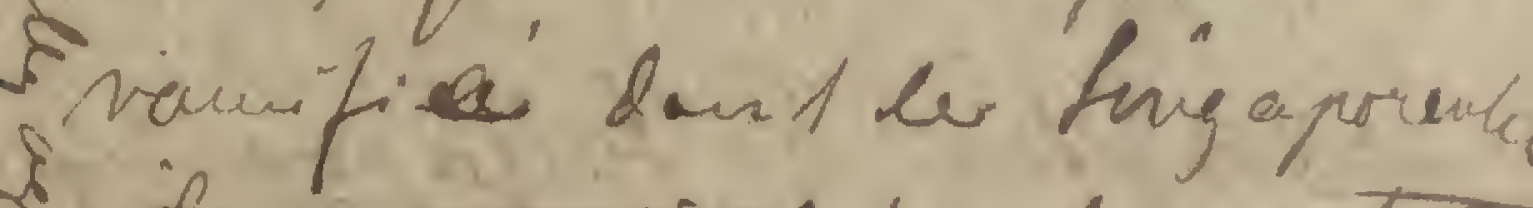
if if a aulf det autry jetithy rfferency dant let endopicy Qef flewis.

's ne felicite anee vans pow let progres quen waus faity parr wive flowe qénero ¿. la retrquile malands.

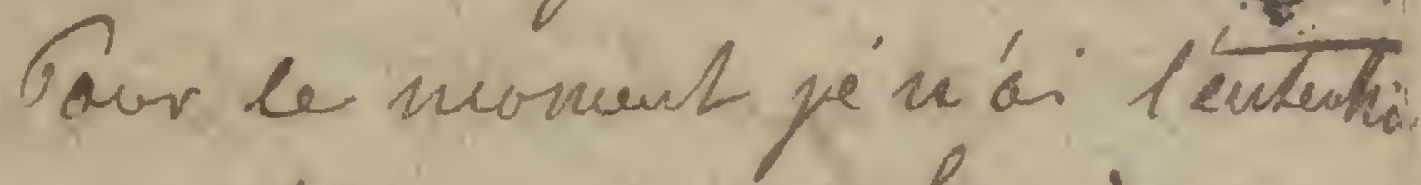
de putrior que les Lepystearya hivout en any quelquine - que vaut animeg bóvair deter unine jé und létudierai ane. Maitir at blant le cus que, vant lanes trane noville jos lo publiaton on ye nome ques vand aurey bone 
Clova,

THE MOUNT,

LEATHERHEAD.

TEL. LEATHERHEAD 202.

8 Yan. ' $\$ 33$

luy dsar fortatam.

d) arefratucale ype on the

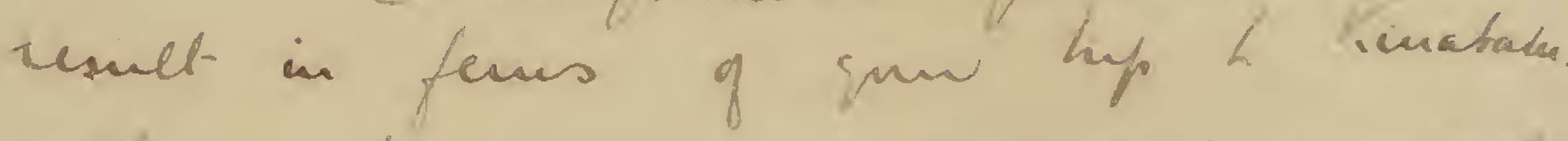

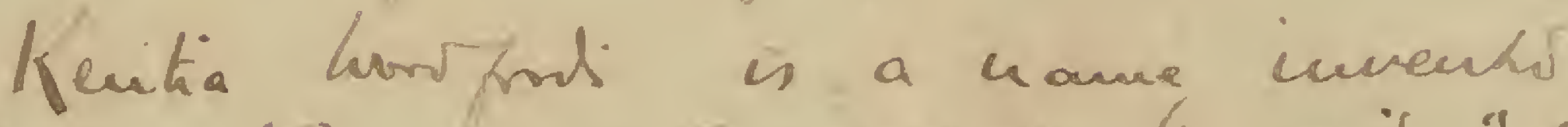

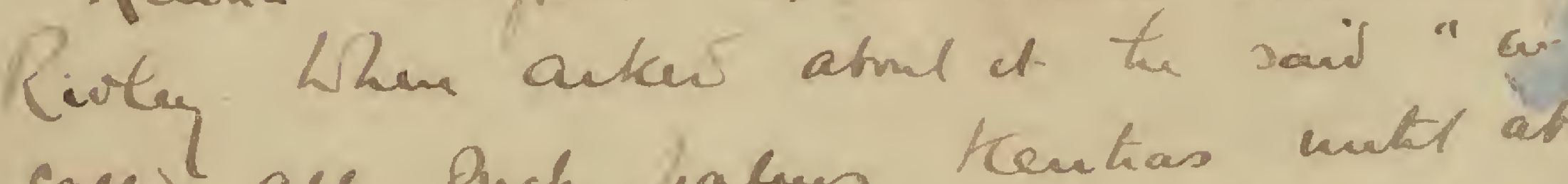
caced ace puch paluess thentras muts ath

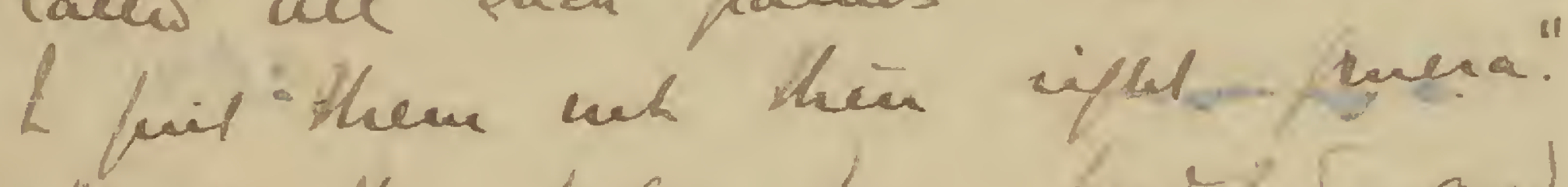

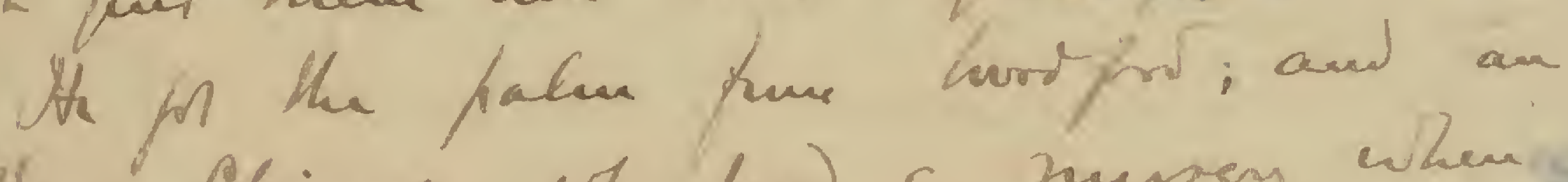
thing Clicang. Who had a menvery where

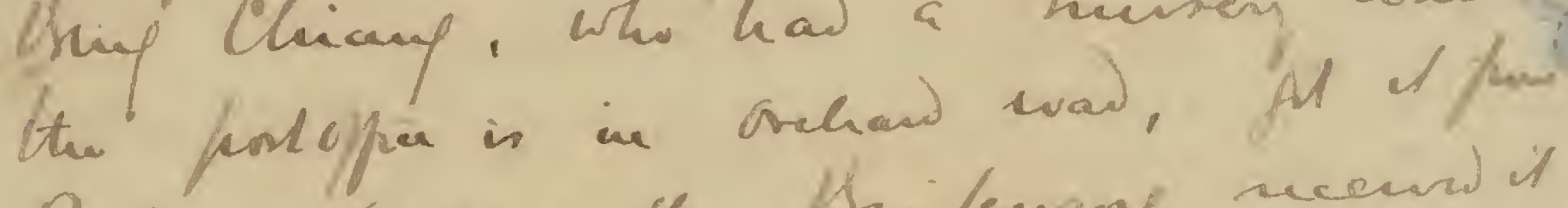

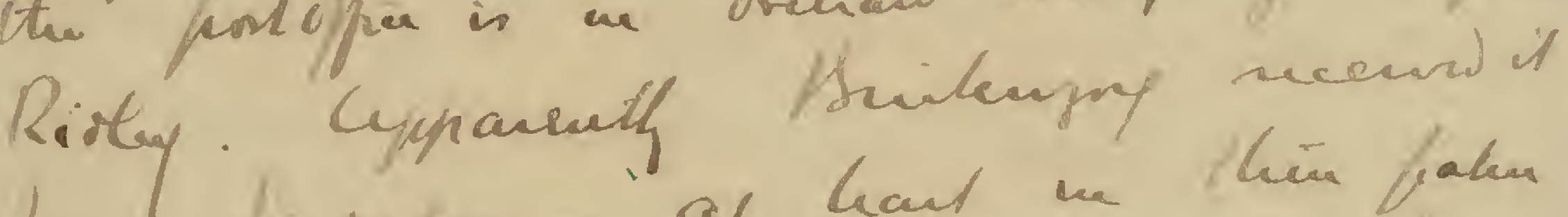

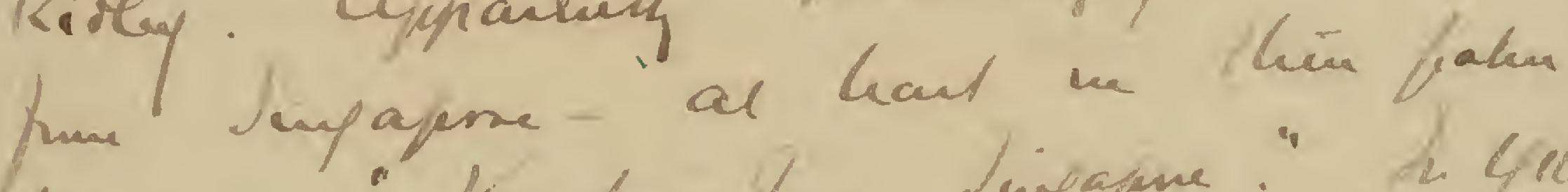

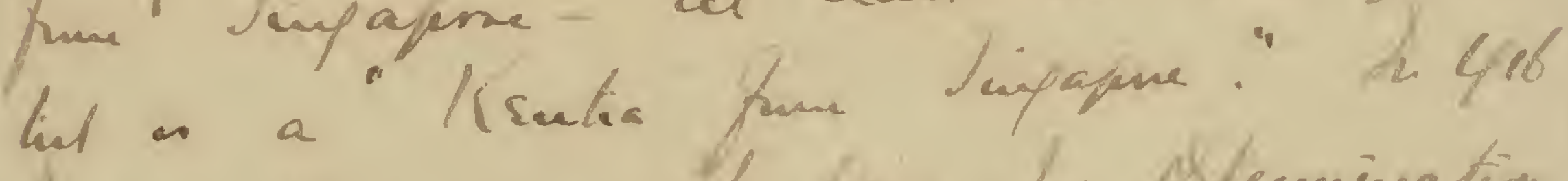

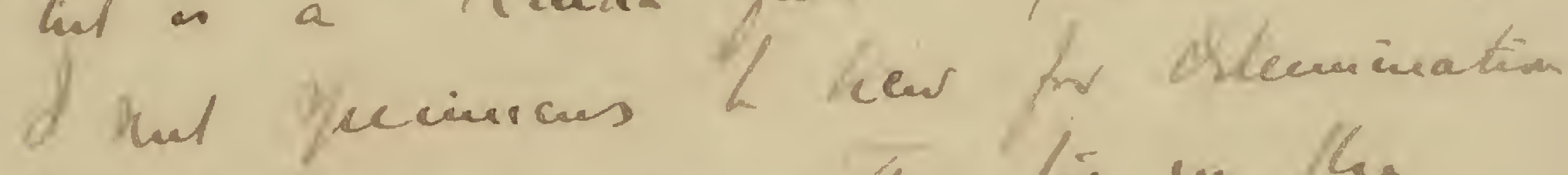
hat 12 ho answes. Thy lis we the

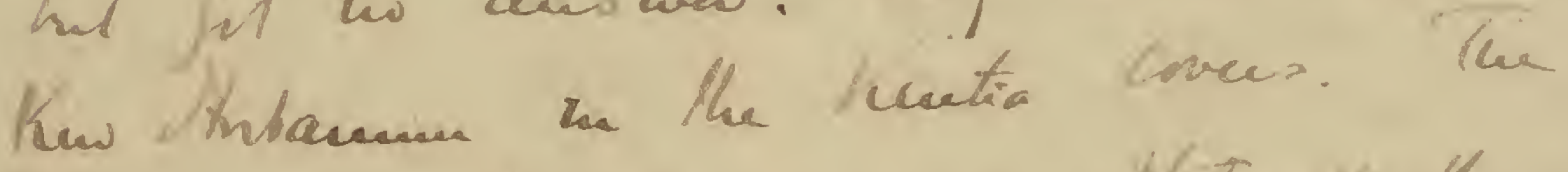

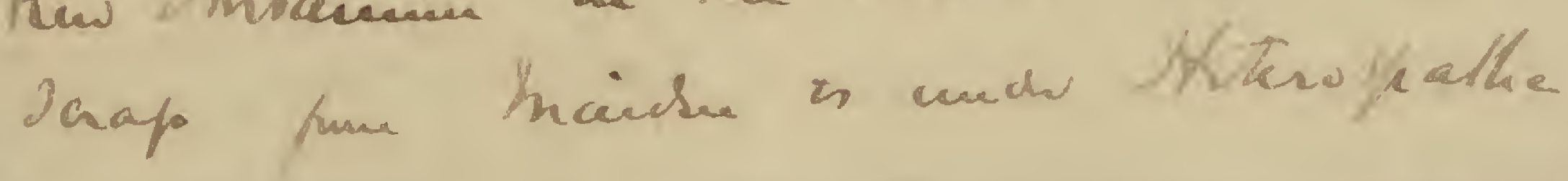

B1. 4518 


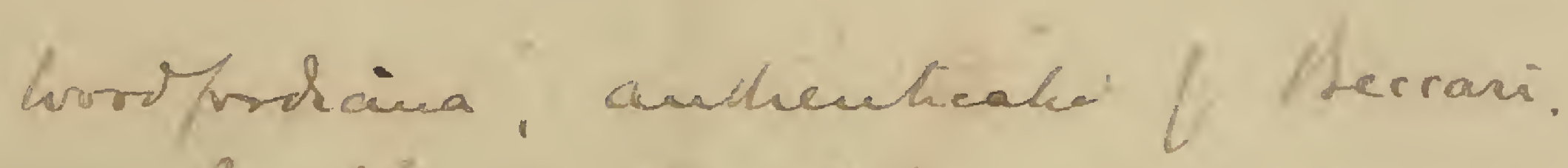

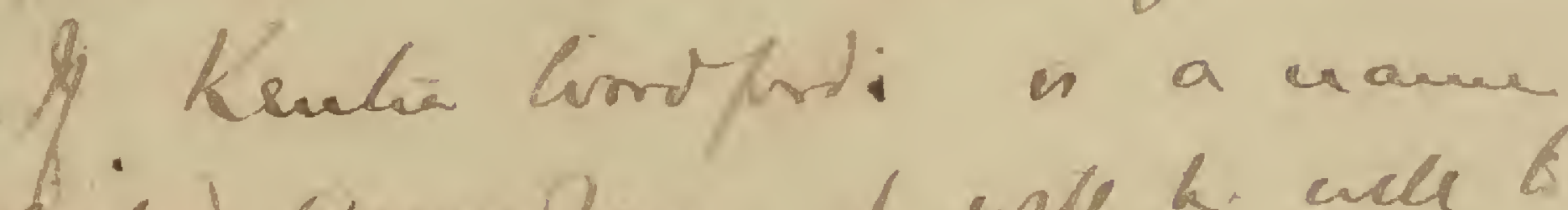

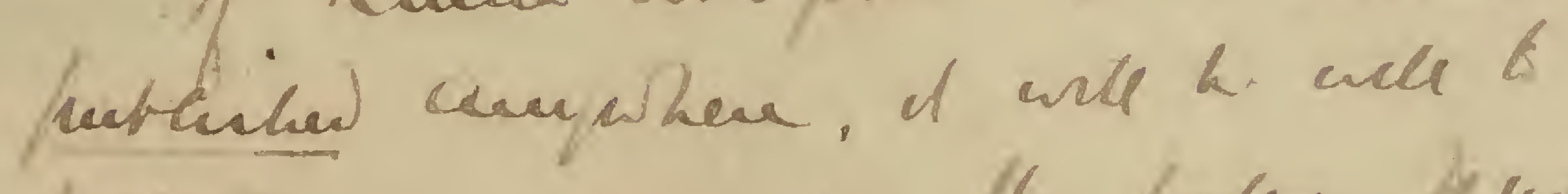

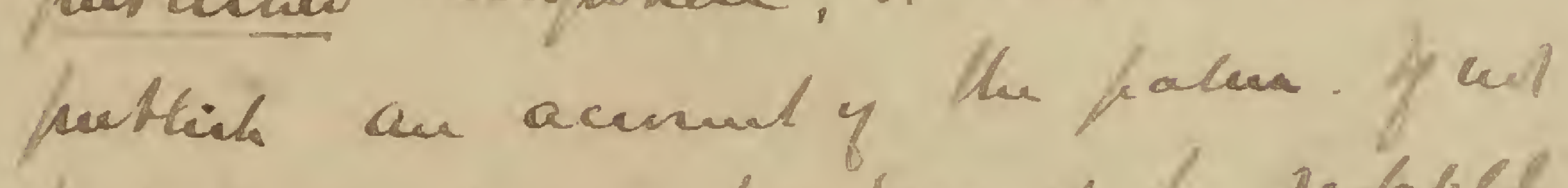

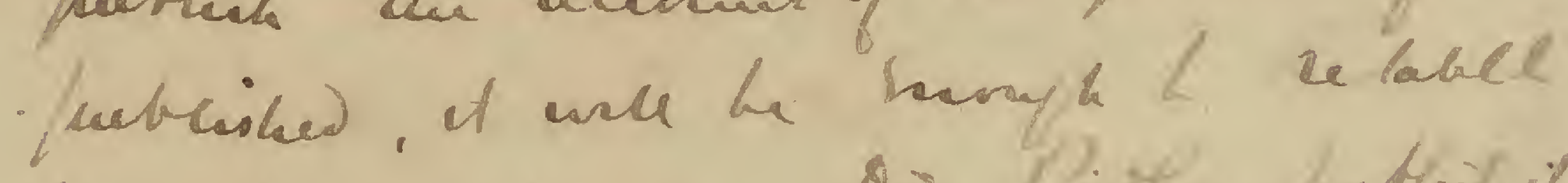

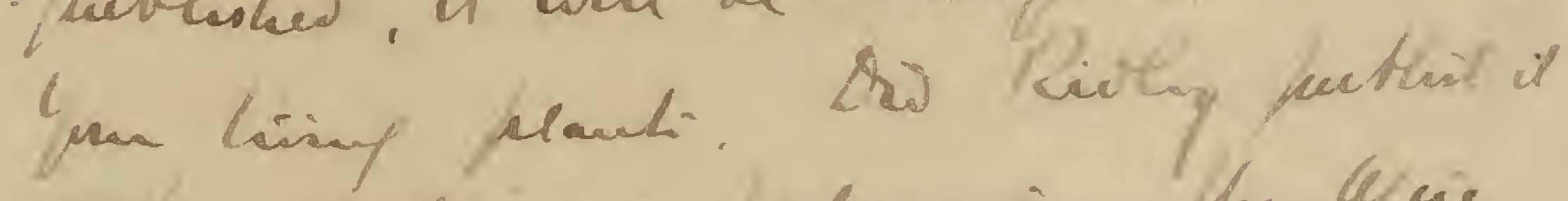

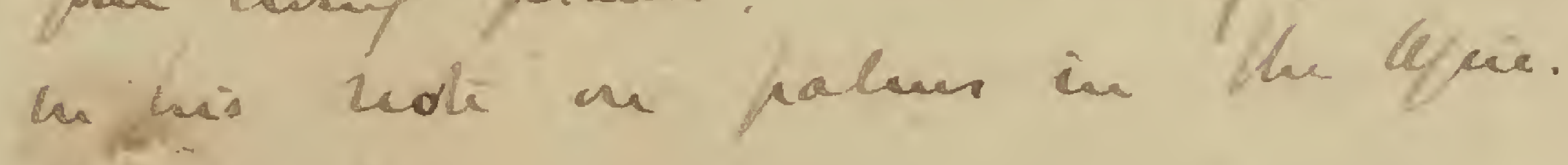
thellutisos?

whom cherlads: wate for your me.

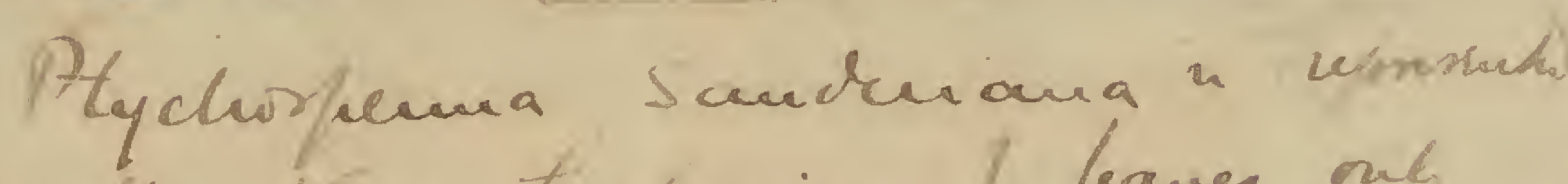

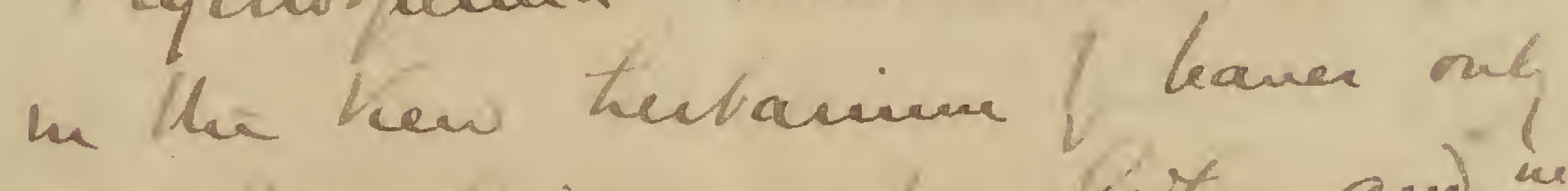

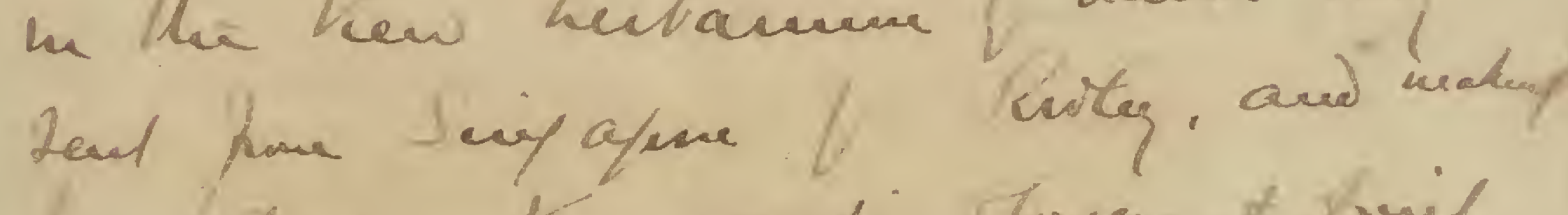

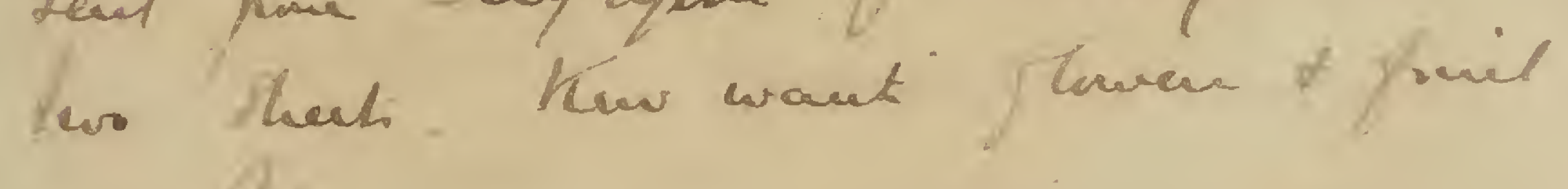
Quens serey

C. Thu thenkach ivter Petolivion is Metaravia/ and

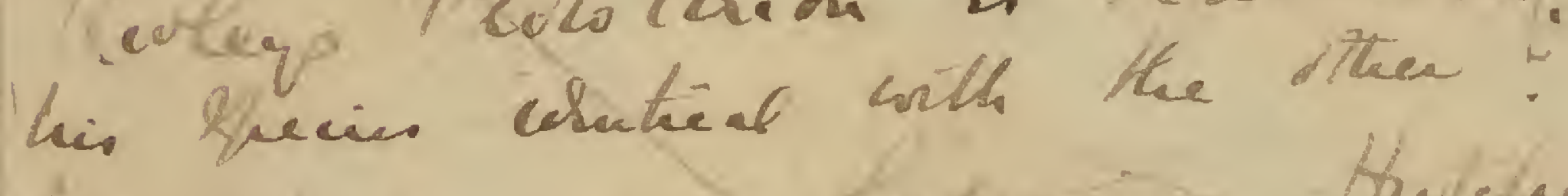

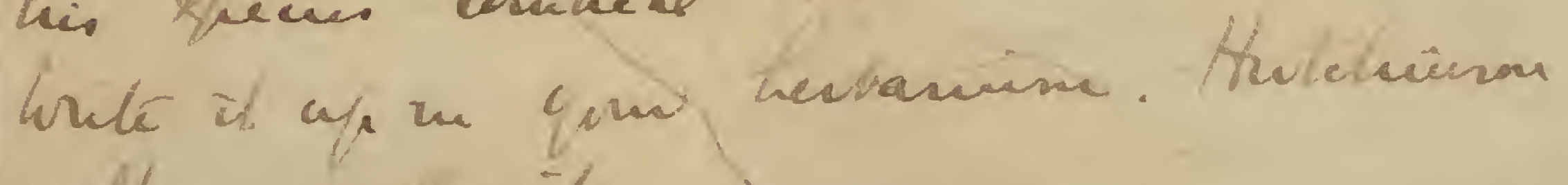
" tha dur waits 


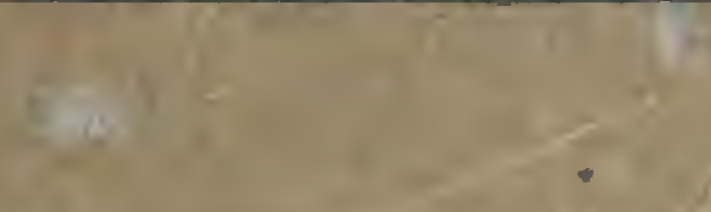

Clova, The mount,

Ieatherhead, Surrey

"Fa. April, 19.32

My doar Holttum,

: I have been torring at Remhanus todey; and I.

find Ker very poor in fir eastern materiaj. Theie id

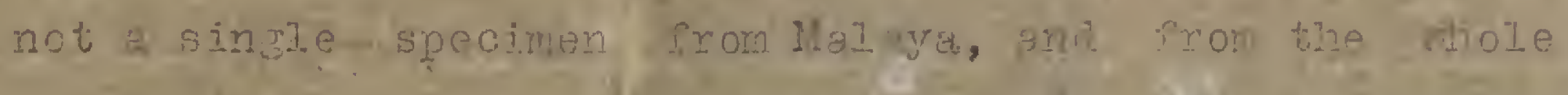
of Wolujsia only throe sheots from Java, wo dun a the euch other.

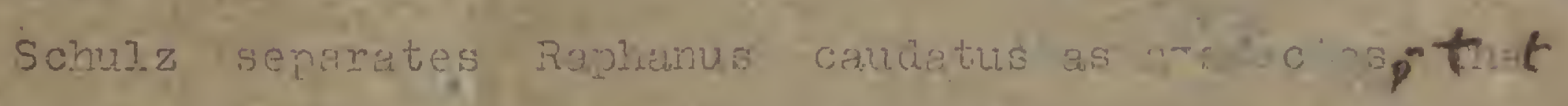

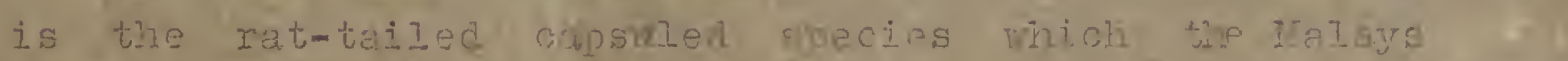
grow as a vecatable, - from the true radish or R.

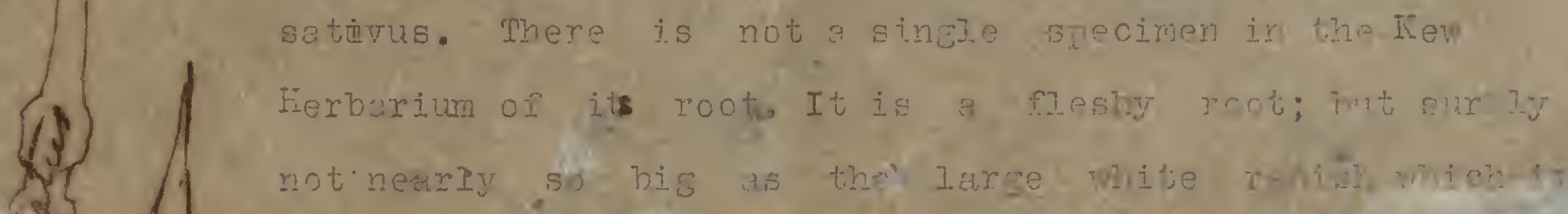

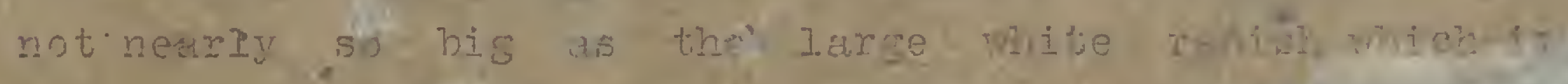

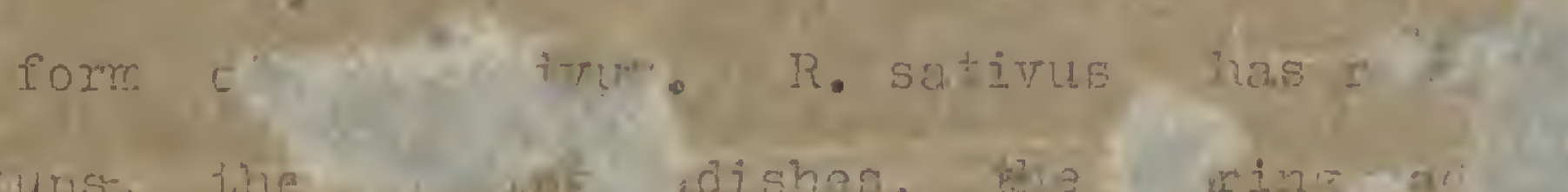

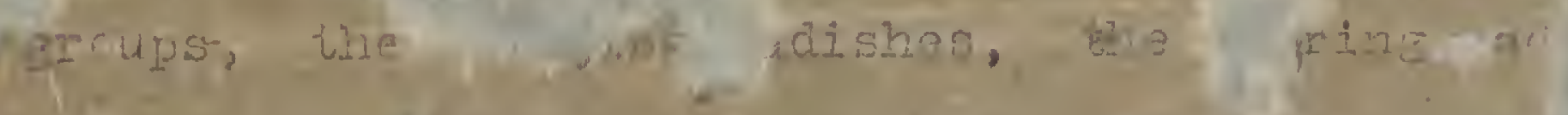

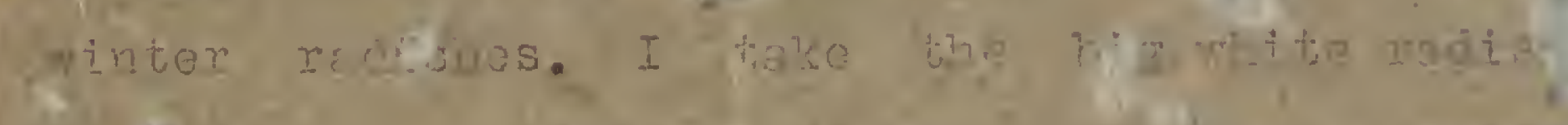

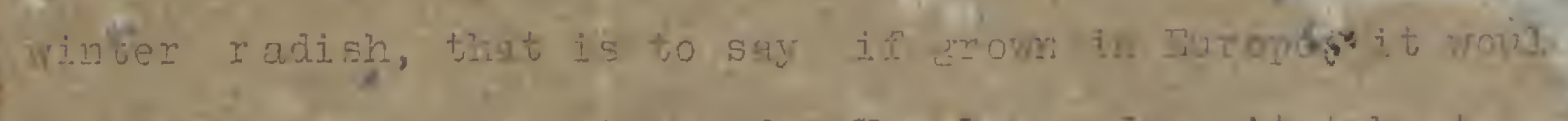

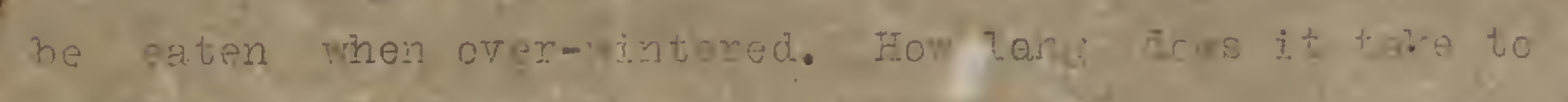

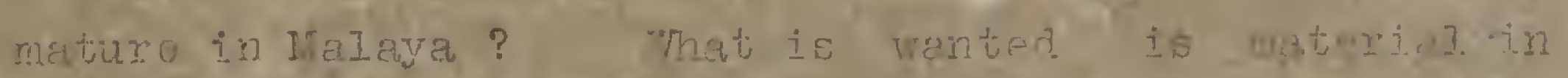
Which the nature of the root is rerlonstriated in Brgoci-

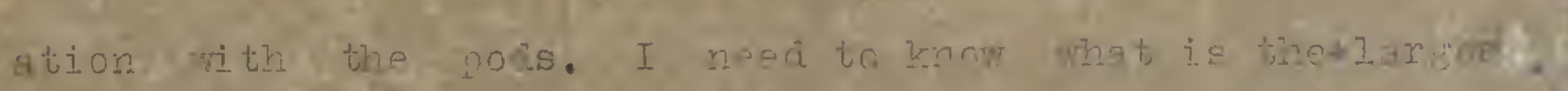
size which the root of canciatus aroeches. Can you help me at, dis.?

Do the chinese import seed ? I pxi pt so. At last ae ure getting solithing rike snrine.

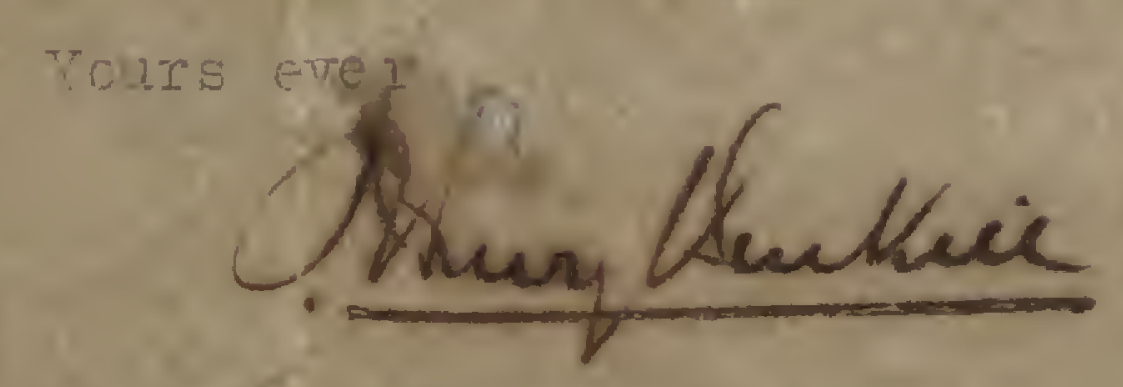

hanas
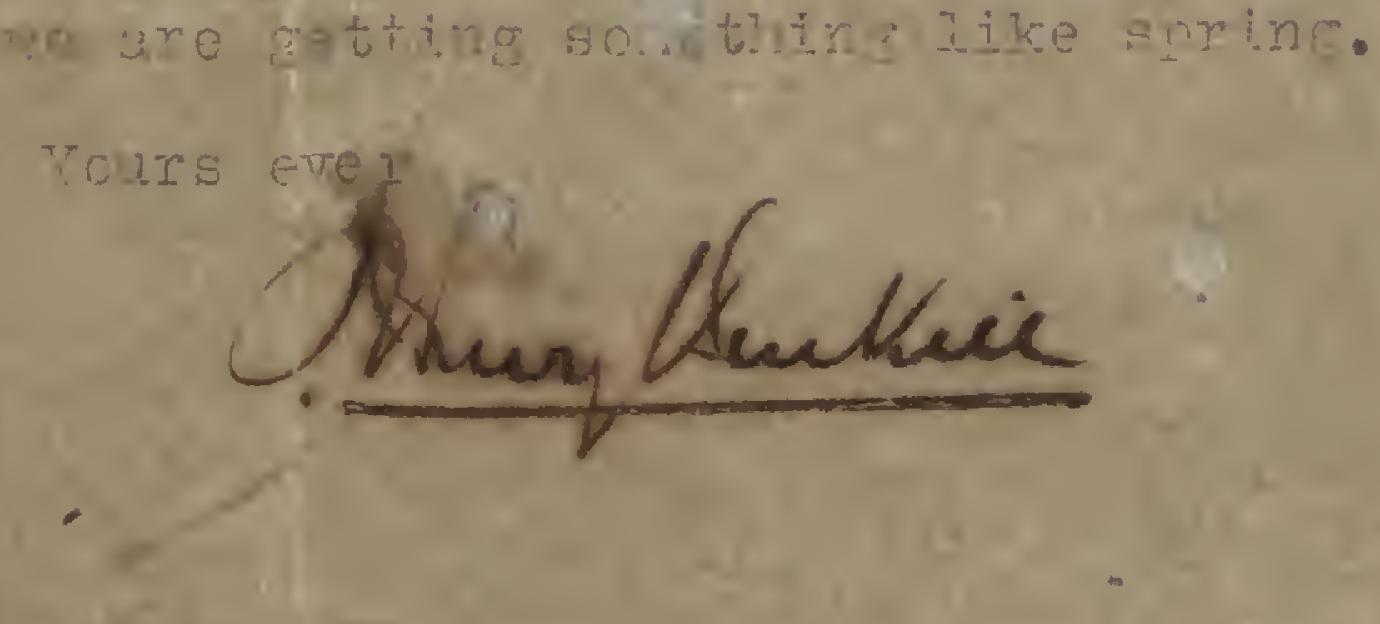

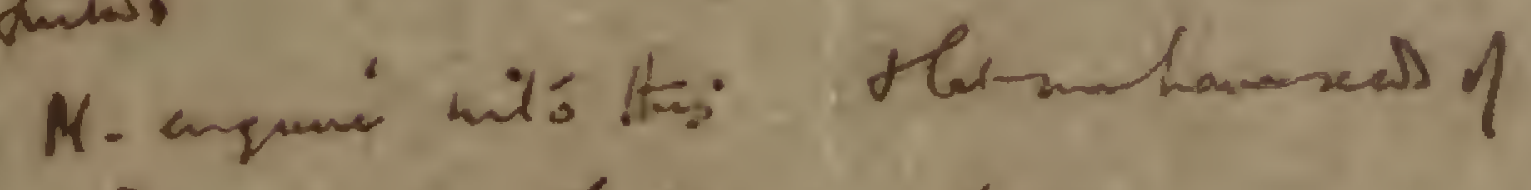


Sandoricum Rumphii; seque de eadem in Dictionario encyclopedico acturum esse nomine Hantol, ubi et meam floris explicationem additurum. Ut ergo Botanici possideant huius generis fructificationem omibus numeris absolutam, tabulam 203 delineavi. Obs. II. Joannes Rajus 1.c. in descriptione plantae a patre deorgic Camello communicata dixit: Hantol Indid, Maxk Hispanis Santor. Arbor alta est et basta, trifolia, Flores perpusilli, bene olentes, subflavi, ex quinis petalis, et calyculato fructus rudimento constantes, racematim congeruntur. Poma magnitudine, figura, qualitate, sapore et colore Cytonia ementiuntur, verum minus lanuginosa. In eorum meditullio quatuor aut sex fusca, cornea, magnitudinis maiorum favarum ossa, membrana tenaci ocoperta, et carne alba, mollecula, grate acia, tomento infaerente obvoluta. Ex. hac Rob grati saporis, Rob mororum vices gerens paratur. Ex succo vero pomi, syrupus qui Cytoniorum substituitur. Poma de reliquo passim et copiose sacchato condita pro bellariis asservatur.

Huius plantae nomen ab Hispanis datum, Bantor scilicet, ternatensi congruit, quod juxta Rumphium foco citato est Santul, et Sontoor. 
Sandoricum Cav. Diss. VII. (1789) 359.

Calyx monophyllus apice quinque dentatus. Corollae petala quinque linearia patula, basi tubi staminiferi adfixa. Staminum tubus erectus, longitudine corollae, apice decemdentatus; antherae decem basi internae interiori dentium adfixae. Germen globosum, superum; stylus simplex apice globoso-sulcatus;stigmata decem brevissima, revoluta - Fructus: drupa sphaerica pomiformis, foeta nucibus quinque ovato-compressis, apice acoutis, basi bivalvibus, monospermis. Semina amygdalina, hilo arcuato. Sandoricum indicum (Tab. 202, 203).

S. Foliis ternatis ovatis cuspidatis alternis; floribus axillaribus racemosis.

Sandoricum Rumph. Amb. 1, p. 167 to 64 . Hantol Rai. hist. 3 plant. Luzon, p. 54 n.9. Camelli tab. 136 inedita. Caulis arboreus, cuius cortex cinereus et glaber; lignum vero, centrale praesertim, rubrum; huiusque odorlatomaticus rosmarinum referens.

Folia alterna, longe petiolata, ternata; petiolus communis tomentosis, canaliculatus, basi incrassatus, quatuor pollices longus, in cuius summitate exstant duo folia opposita subsessilia, inter quae tertium assurgit, petiolo bipollicari sustentatum. Haec sunt ovata cum acumine, integerrima, uninervia, nervo ramoso, supra glabra, subtus tomentoso-ferruginea. Stipulae nullae.

\# Flcres parvi, racemosi; racemis axillaribus semipedalibus, ramulisque alternis multifloris. Calyx monophyllus urceolatus. Tubus staminum rubescens. Fructus initio acidus, deinde alliaceus, cuius carg tenera albicans.

Jabitat in Philippinis, Molucis, et passim in India Orientali V.S. apud DD de Jussieu et Lamarck PObs. I. Plantam delineatam t. $202 \mathrm{sec}$. exemplar Jussieuanum communicavi D. de Lamarck, qui humanissime mibi fructum ostendit, huiusque prima delineamenta Sonneratiana, dixitque illam esse Saubarieum Rumflii 
The majority of the changes in the nomenclature of the Indian plant and for the matter fact, of the other parts of the British Colonial Empir are mosty due, not so much to the priority rule, but to the fact that Hooker and his colleagues at Kew adopted what is known as the "KEW RULE" in preparing the Colonial Floras.This rule not only maintained that the correct name of a species is the first combination under the correct genus, but also permitted one the use of later homonyn or even a synonym as a legitimate name for a species simply because the particular binomila appeared to them a better or more appropriate name to the species han any other, ormen older, name. It even permitted one to accept a later interpretation of sas a binomial to the prejudice of the original one, and irrespective of the type. This rule katx was therefore responsible for much confusion and friction among botanists, though it is the influence of Hooker and Dyer, be so to say

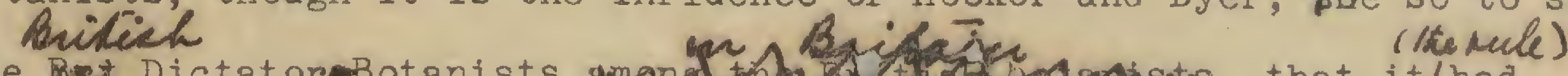

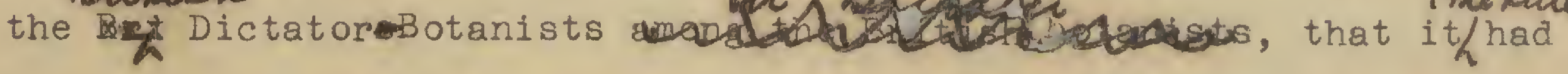
been able to axpres carry on its existacerso long. It is only after these dictators ceased to exercise any inflence that the British Botanists began to realise the false position into which they were led by their former botanical leaders and so cols they began to follow the majnrity of the botanists and to support the priority rule, and later they in $1,3 /$ gave their support to the inclusion of the provisior in the inter ional Code governing the/nomenclature. But even prior to the Vienria Rules(1905), the majority of the systematists on the continent, in America and even in India were in the favour of the practice of interpreting a binomial in its original sense and giving preference over others to the earliest specific epithet.ffence, it is obvious that those who have become long accustomed to the Florgs emating from Kew resent that they should be asked to discard the wrong interpretations and to accept instead names and interpretations which are really older and legitmate ones. The schism that is now threatened by the British Foresters is no doubt an outcome of wrong conceptions on the matter, for it is $h$ illegitimate interpretations and usfre that they are trying to legalise on the plea that they had become very long accustomed to them. Should we revert to the kew rule again, it 
is to be feared that the majority of the botanists in America, Germany etc. may revert to their old attitude and maintain thernse?ves rather aloof from the British Botanists and thereby contribute to make the plant nomenclature more burdensome. It well then

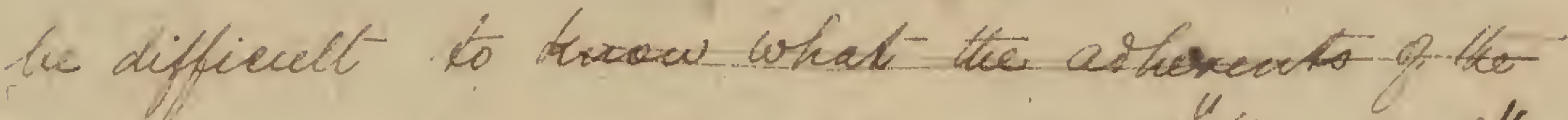

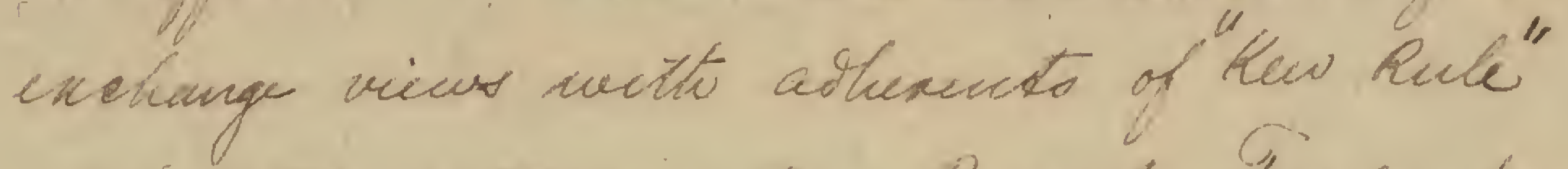

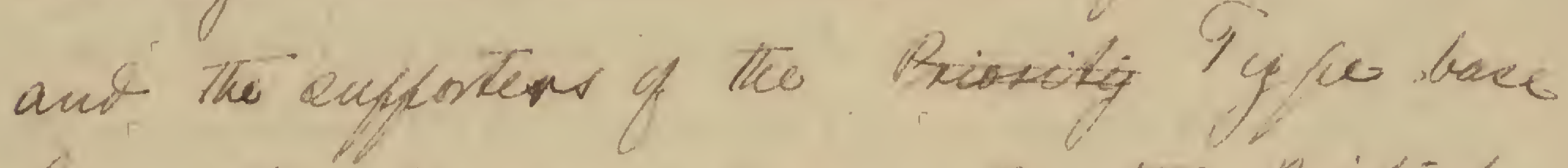

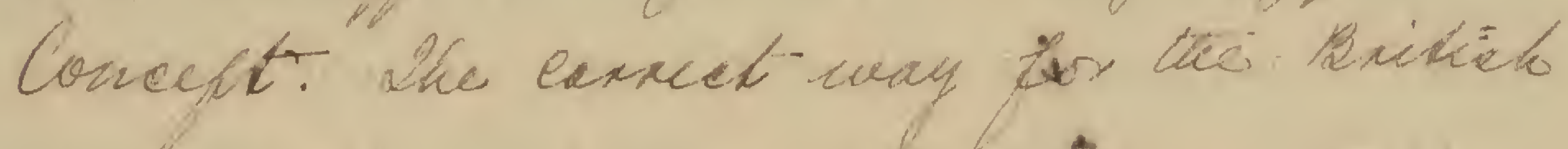

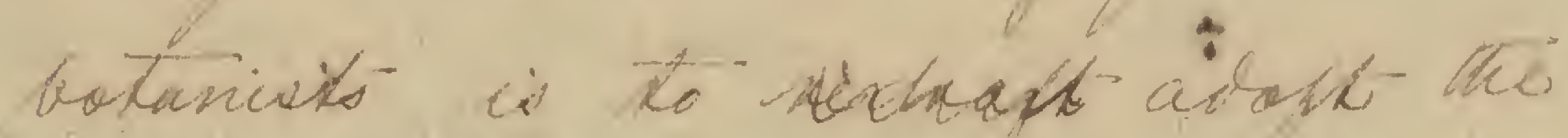

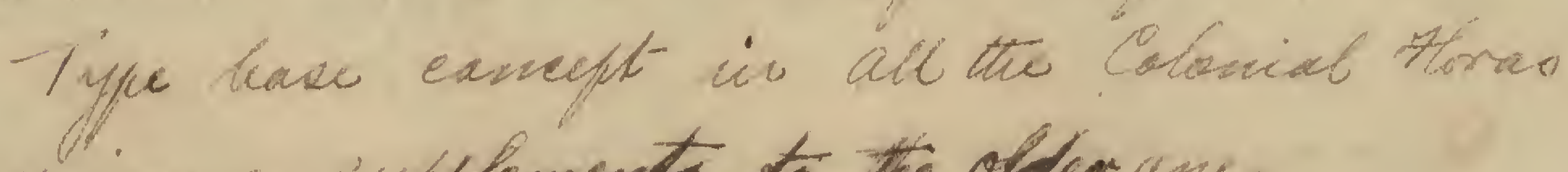
Q is cue supplement o to the oldepane.

ix ins ant

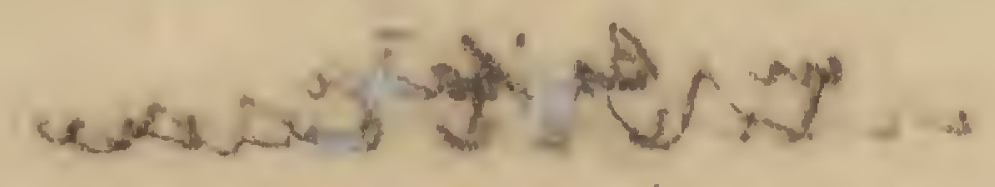

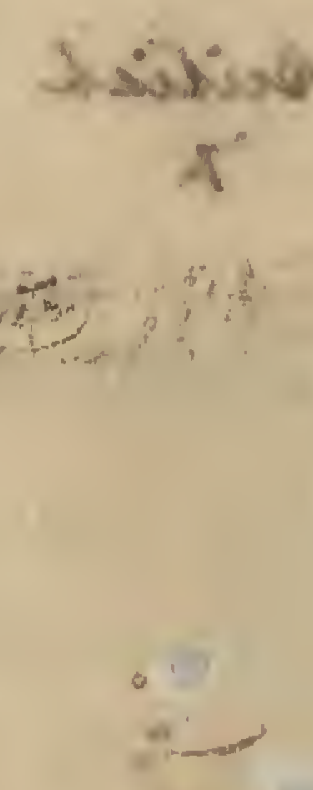

Wal 
Port Alberni,

Vancouver Island, 16.7 .41

Dear Furtado,

After a very full month of travel, I am now waiting for my ship to sail, and want to write you a few lines.

At the Arnold Arboretum I met Croizat, but did not have the opportunity of discussing types with him. I find that he had a legal training, which may complicate his outlook on the subject:

At New York I met Dr Gleason, who spoke of your letters to him, and appreciatively of your work on nomenclature. He said he did not think you would have any seriois difficulty about the colour question if you come to New Youk, though you might have in the south. I am sure he would help you in any way he could. He is a very alert and also kindly person.

At Washington Ir Maxon told me that they did not have the Gardens Bulletin at the Smithsonian Institution (the Division of Plants of the U.S.National Museum), though they send us their Contributions. They said that the Bulletin went to the Dept of Agriculture. Probably we should send it to both. We can discuss this when I come to Singapore. There seems to be some needless rivalry between the systematic work of the Dept of Agriculture and of the Smithsonian.

I met Dr Foxworthy and Dr Copeland at Berkeley. The former is continuing his work on Diperocarps, chiefly bibliographic, and

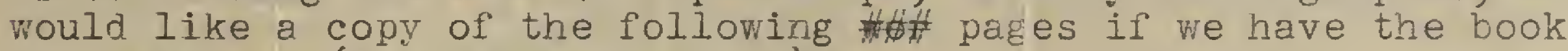
in Singapore (I think we have it):

Lanessan - Plantes Utiles des Colories Francaisea, 1 (1886) pp.297-302, so far as they refer to Dipterocarpaceae.

Will you please mention this to Mr Corner, and send the copt to Dr Foxworthy if possible (762 Arlington Avenue, Berkeley, Cal.)

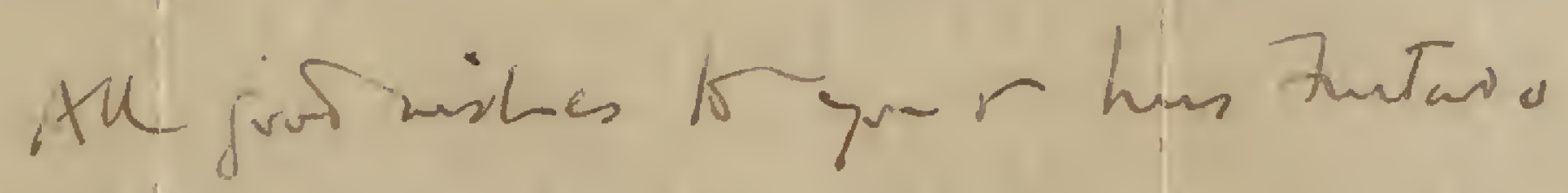


Na.

Sir,

I have the honour to acknowl edge the receipt of your letter No.7 in $422 / 31$ dated 22nd July, 1931 and to inform you that steps will be taken to secure the Rhodesia

Agricultural Journal and the Bulletin du Syndicat des Planteurs de Caoutchouc through direct exchange as suggested.

2. The throe cases of periodicals sent have been received.

I have the honour to be, Sir,

Your obedient servant,

Iibrarian \& Translator, Department of Agriculture, S.S. \& F.M.S.

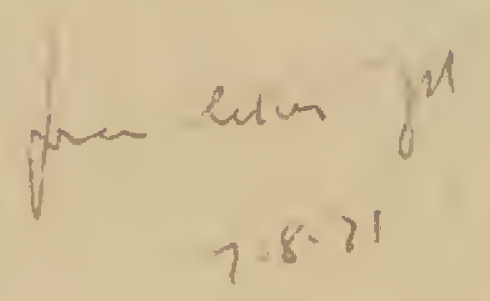


Sir,

In reference to the second parabraph of your letter of June 25 th (concerning the transfer to your library of certain periodicals) I have the honour to enquire whether it would be possible to arrunge an exchance of the Nalayan Aericultural Journal with the two periodicals you do not alrekdy receive (the rhosesia Agricultural Journal and the Bulletin du Syndicat des Planteurs de caoutchouc). If such direct exchange is possiole, it would relieve me or' the necessity of sending the Gardens Bulletin; vut if you carnot smange the exchange I will continue to send the Gardens Bulletin and have the two periodicals in questiors forwarded to you. I note that you already receive the Buljetin de la Chambre and Agriculture du ronkin.

I have the honour to be,

$$
\text { Sir, }
$$

Your obedient servant,

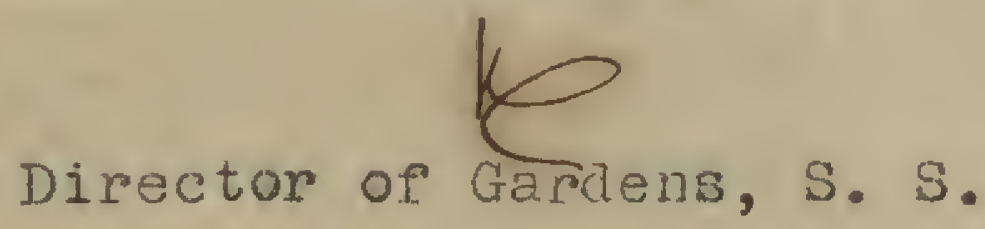

\section{Librarian,}

Department of Agriculture,

Kuala Lumpur,

F. M. S. 


\section{DRAFT}

Fai: Copy signed Ły

To

\section{Lit zenion} ue Deprof Africaltive

$22.7 \cdot 31$

Sir,

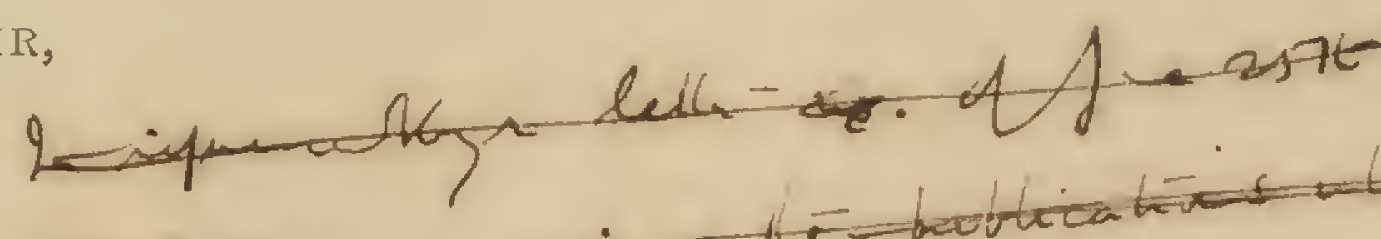

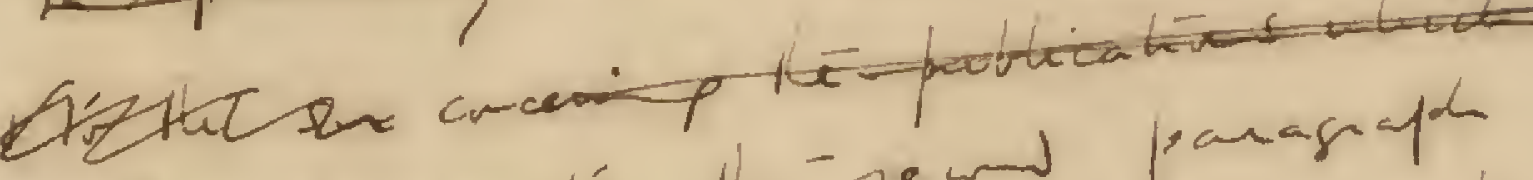
In sefuerae i's the sewed paragraph bellor- 1 grae istit (anceuring the or bivere of centain revisoticals)

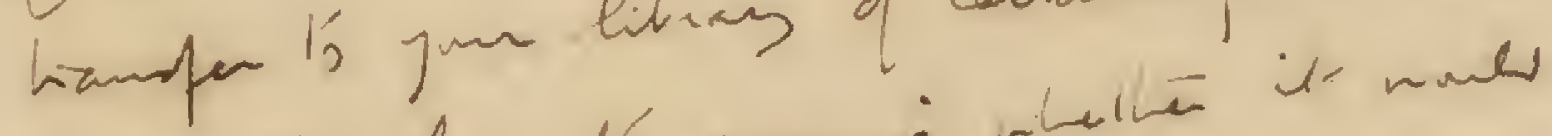

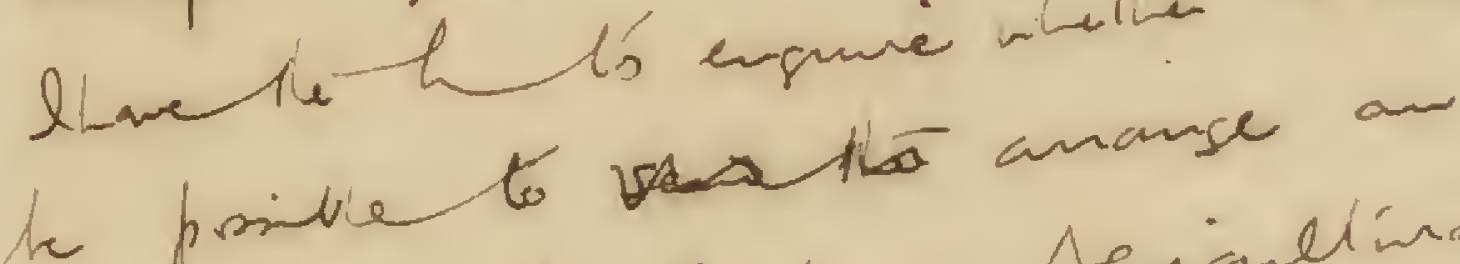
te pristere Aprianlínal excharge 1 the halas an Ano periodicabs for Jonnel wite te knowar. do wh' aheady receive the Ku- Bulletin

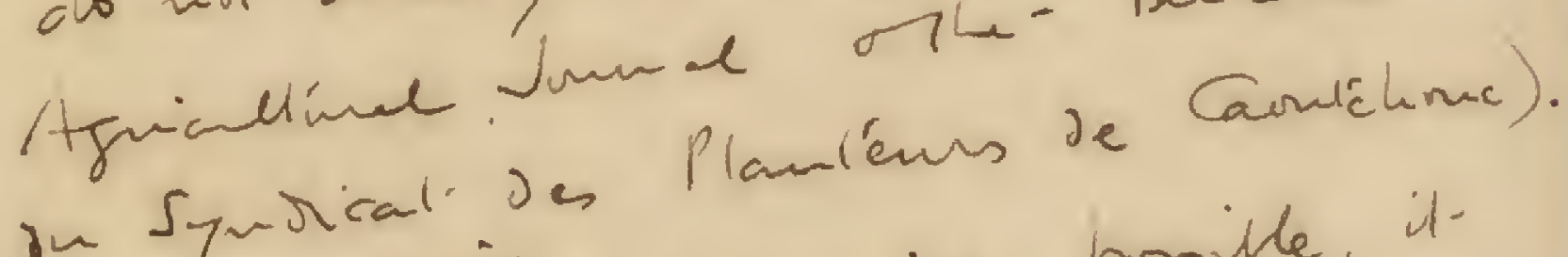
I puch ariexchange is porsible, it would shicue me die-neassits sendif the Ganams Bulletin; hes if Yo cannt anarge the-exchange t

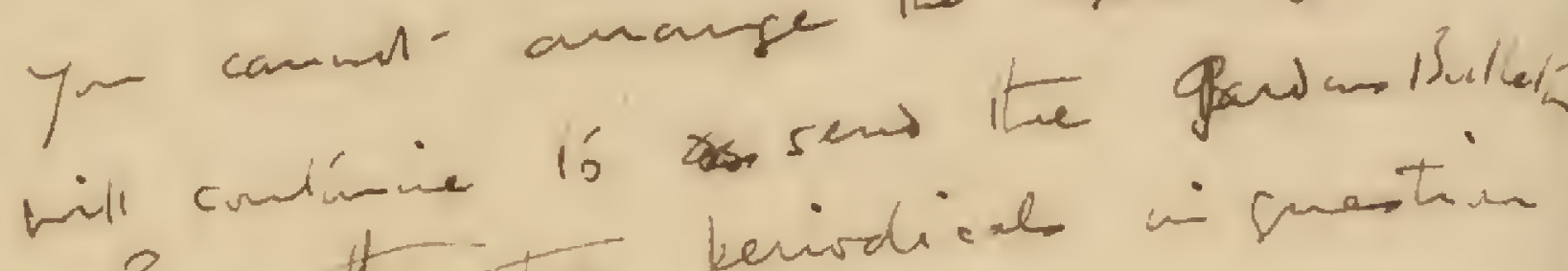
othare the tho perivdical in suestion Gurandor lityoum.

toe witure ter

Insce that $7^{\text {r }}$ doracor ahead recenve the Bubletin de la Chanhe

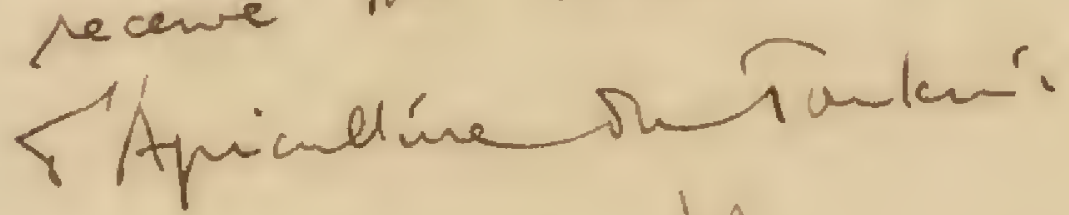


Sir,

In answer to your letter of 25 th June, I have the honour to inforn you that 3 cases containing the periodicals referred to are being despatched to you today by foods train.

I have the honour to be Sir,

Your obedient servant,

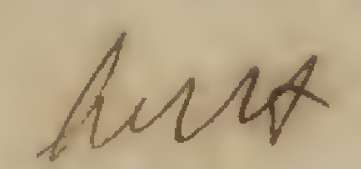

Directior of Gardens, $S . S$.

Librarian \& I'ranslotor,

Department of Aericulture,

Kuala Lumpur,

F. M. S. 
$\mathbf{s}$

G 17

DRAFT

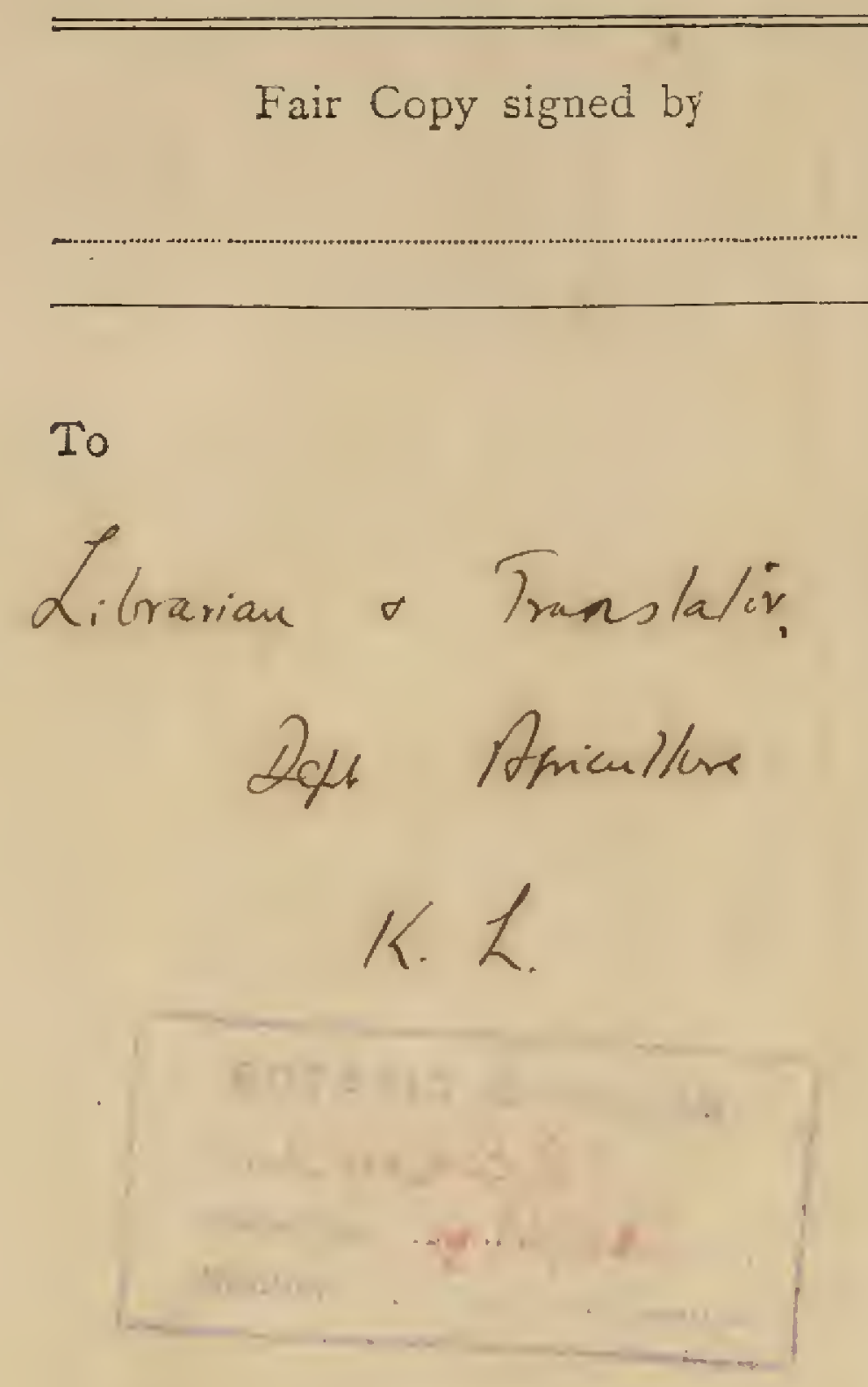

SIR,

la answe 10 jar lette / 25 Jure, i. Ih 2 to inform Jon that 3 cases cautainizy the heriodicels referes to are beij desfatches to in todap by. Gouss train

$$
\ell-7
$$

and 26$)$ 
Na.

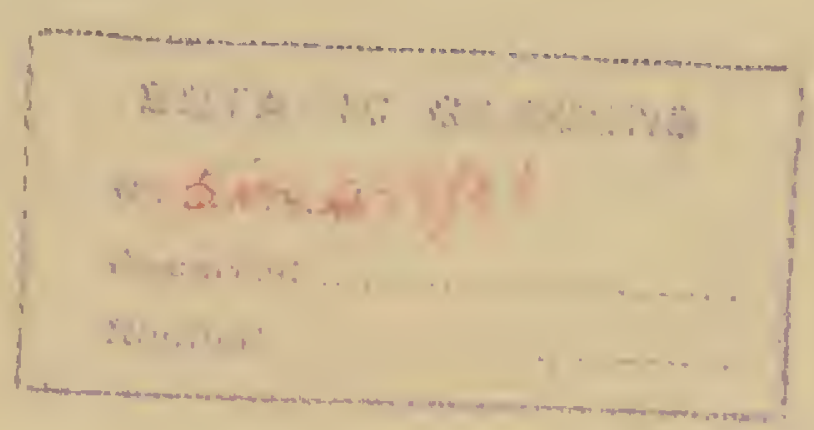

DEPARTMENT OF AGRICULTURE.

STRAITS SETTLEMENTS

$\&$

FEderated MaLAy States.

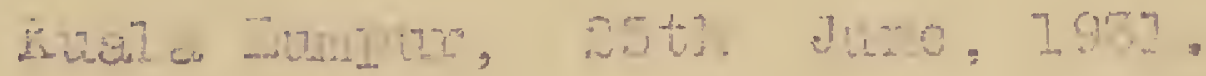

Sin,

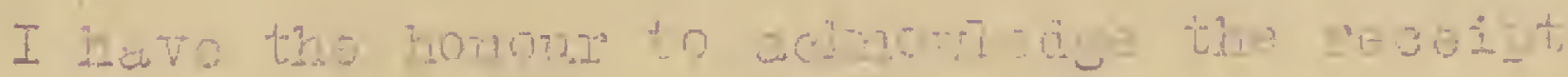

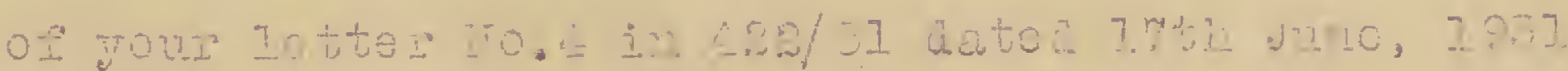

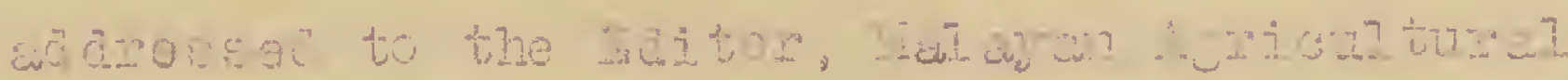

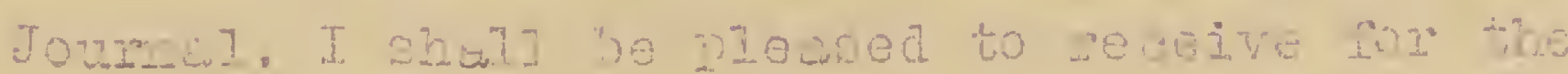

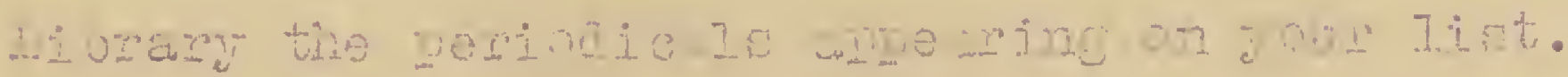

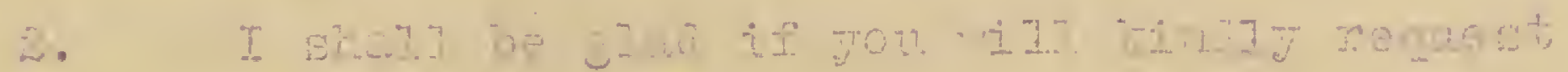

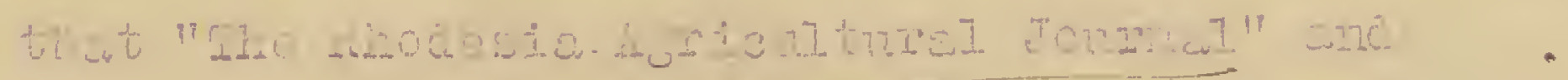

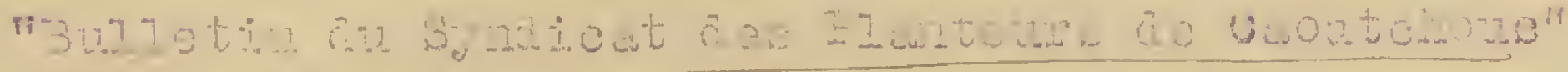

Mr. Furtado

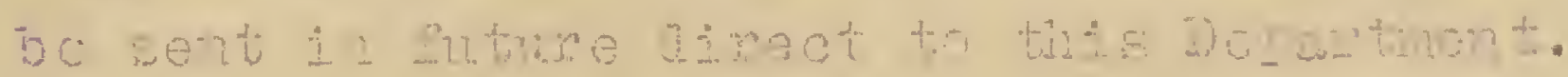

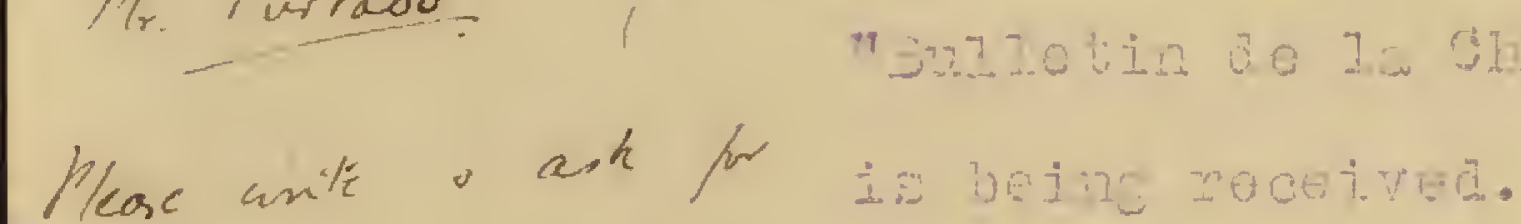

then to be tent disect

to Kuala hump...

$$
\text { / unded }
$$

whe luector of vindeno, s.s.

Jot rid savens,

Bif 1200
I Ierve the bolpug to he, Si.:

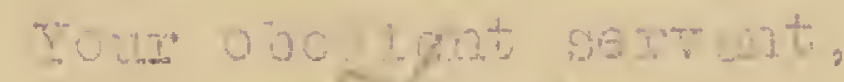

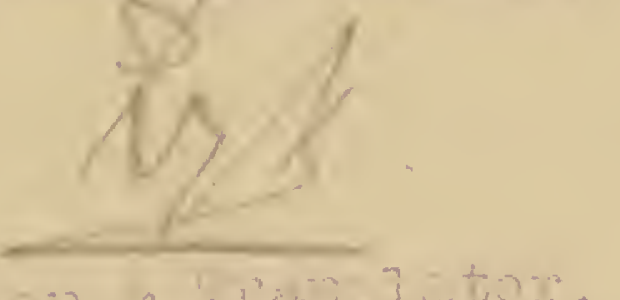

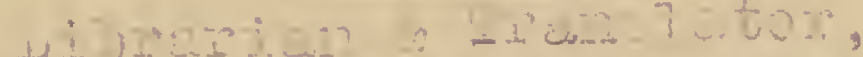

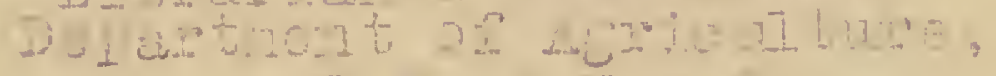

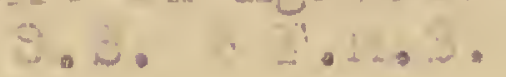

frevinu letion pu. 
Sir,

In reference to your letter of August 27 th 1929 and mine of september $3 \mathrm{rd}$. I regret that the journals concerned have never been sent to you. These journals, being as specified on the enclosed list, are now being packed and I proposcto send them to you on Monday next.

The following 3 periodicals are still being received here regularly as exchanges:

The Rhodesia Agricultural Journal

Bulletin de la chambre a'Aericulture du Tonkin

Bulletin du Syndicat des Planteurs de Caoutchouc (Saigon)

I do not desire to continue to receive these, and propose to write to stop their being sent. If however you do not already receive any of them I will request that they be sent direct to you instead of to singapore.

I have the honour to be,

\section{Sir,}

Your obedient servant,

The Editor,

Malayan Agricultural Journal, Department of Agriculture,

Kuala Lumpur,

F. M. S. 
List of Periodicals sent to Department of Agriculture, Kuala Lumpur.

1. Annales des Planteurs de Caoutchouc de L'Indochine 1911-1918

2. Bulletin de la Chambre d'Agriculture de la Cochinchine 1908-1920

3. Bulletin de la Chambre d'Agriculture de Tonkin 1910-1931

4. Bulletin de Syndicat des Planteurs de Caoutchouc (Saigon) 1918 - 1931

5. Rhodesia Agricultural Journal 1909- 1931 .

6. Journal of the Department of Agriculture, West Australia 1900-1908

7. Agricultural Gazetie, New South Wales 1907-1914.

8. Agricultural Journal of South Africa 1911-1914 (I-VRB)

9. Agricult. Journal of Victoria 1904-1920 (II-XVIII).

10. Agricultural Journal of Cape Colony 1888-1910

11. Natal Agricultural Journal 1904-1910

12. Transvaal Agricultural Journal 1909

13. Greniers' Rubber News I-XI

14. Inda Rubber Journal 1904-1928

15. India Rubber world 1901-1925

16. The Farmers' dournal, Kenya I- VIII (1920-26)

17. Tropical Life (odas)

18. La Hacienda (odds)

19. Le Caoutchouc et la Gutta Percha 1903-1913

20. Bulletin de L'Association des Planteurs de Caoutchouc I-IX

21. Agricultural Jourral of British East Africa I-IV

22. Revista de Agricultura, Republica Dominicana VI-XIV

23. Journal de Agricultura da Companhia de Mocambique I-II (1911-1912)

24. Journal de Agriculture Tropicale 1901-1914.

25. Boletin de Bosques, Pesca i Caza I-II (1912-14). 
s

G 17

DRAFT

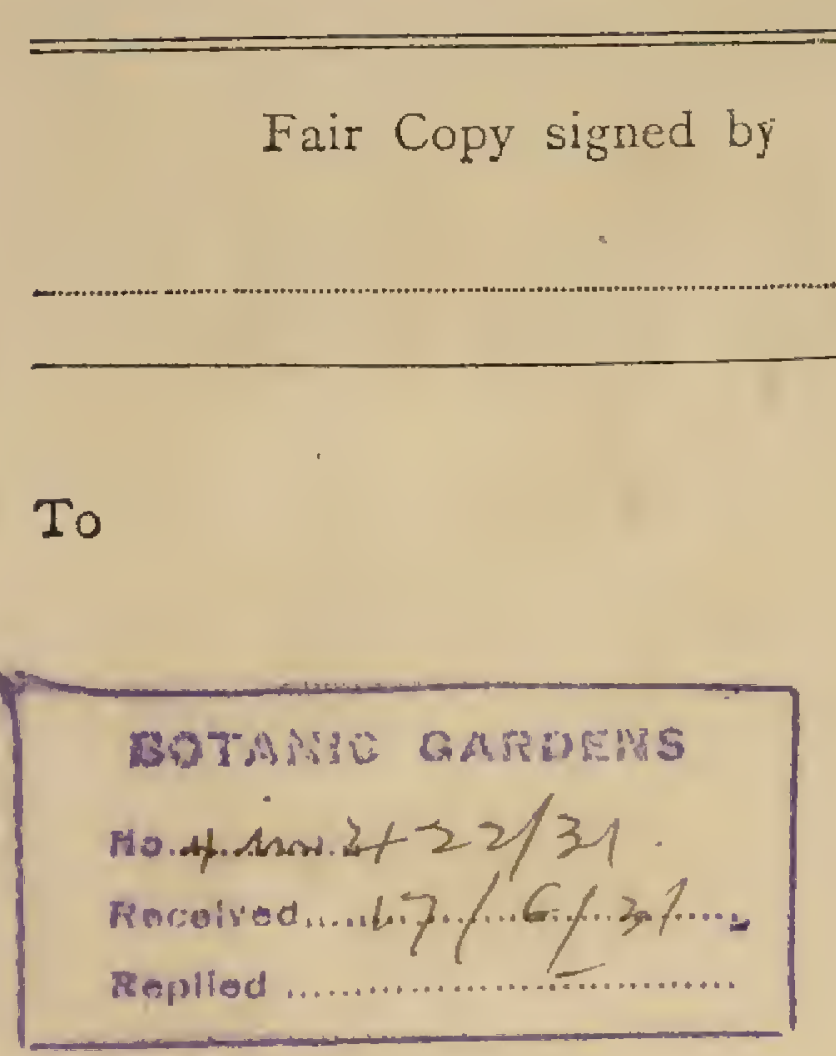

The Zdilin m. 1.

$$
17-6-31
$$

SIR,

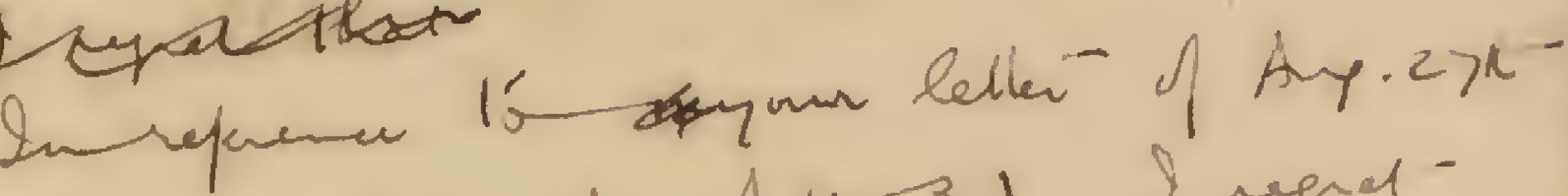
1929 of mmi of sept 3us, I repal -

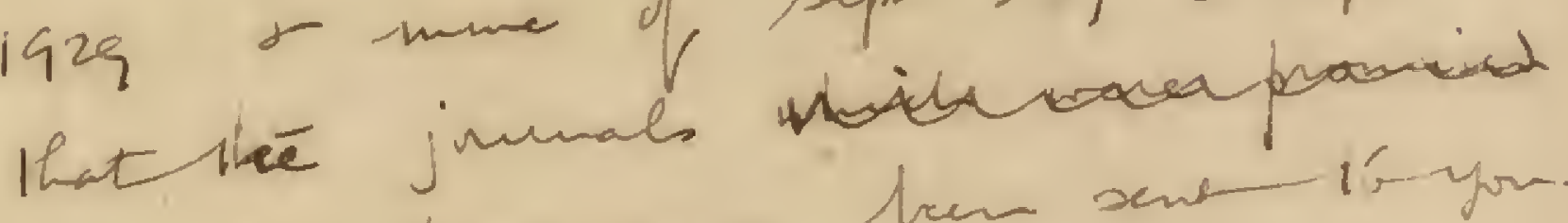
comernes have newar heer sent is your. There joumals, bery as pecipled ar

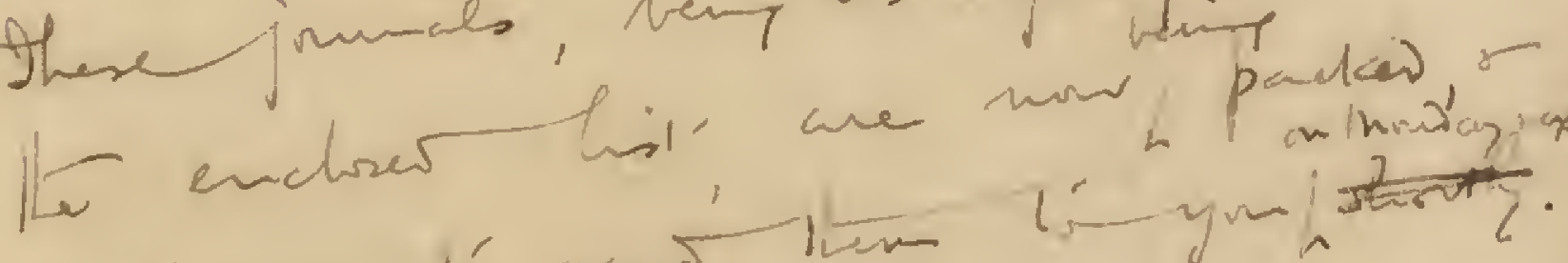

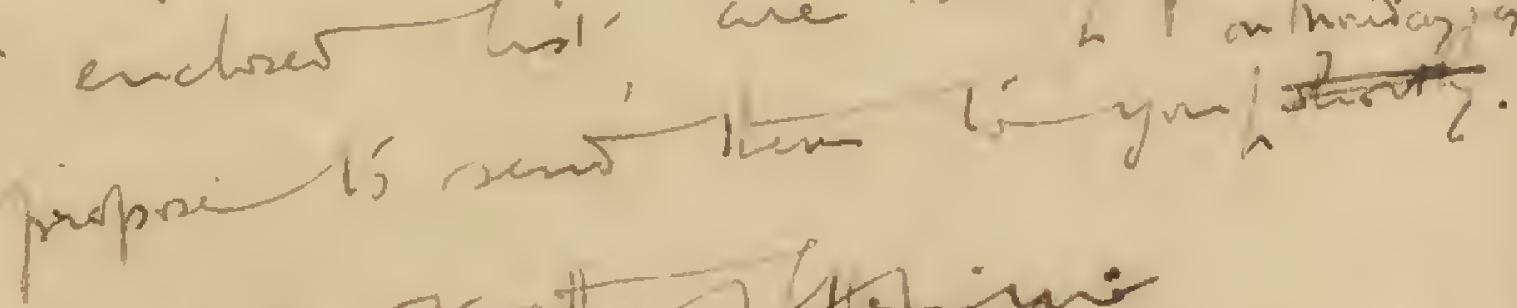

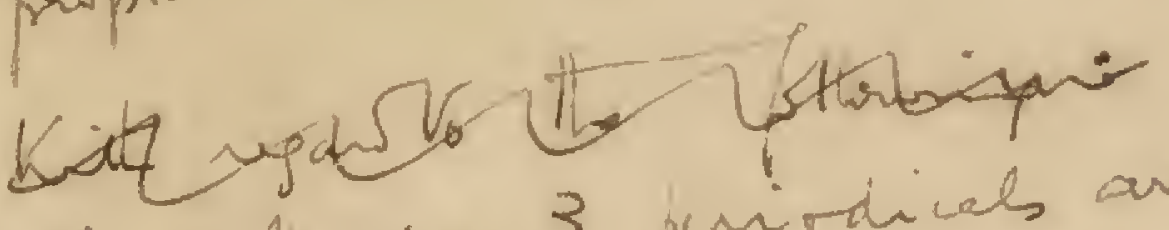
The pllowng 3 periticicals are stit

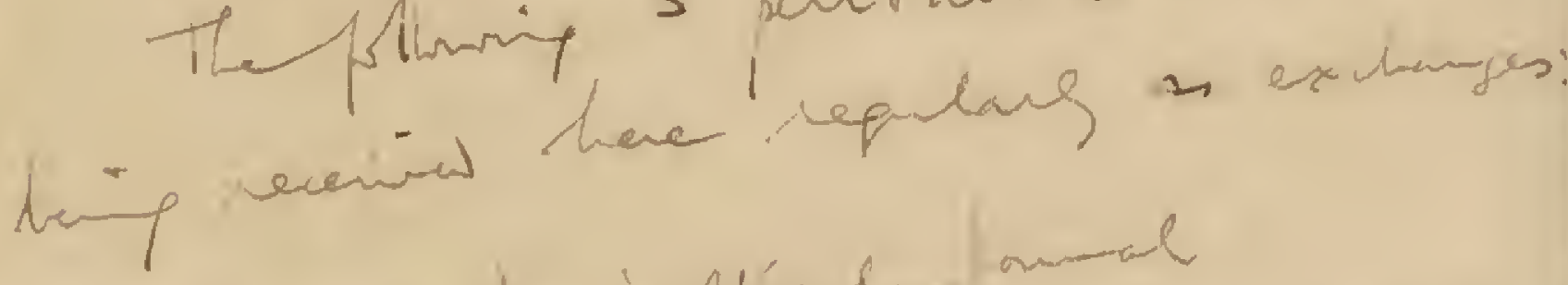
The Rhodenia Agrianlinal amol

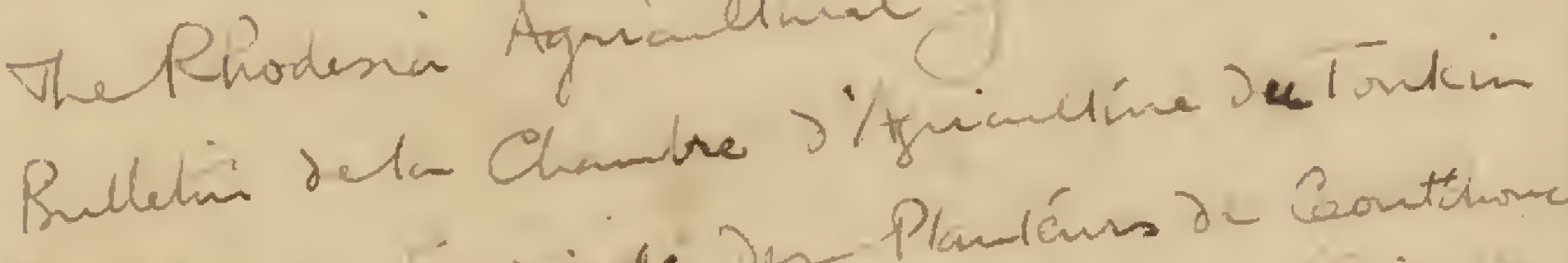
Bulletín on Syparical. Des Plandénes de Conttionse

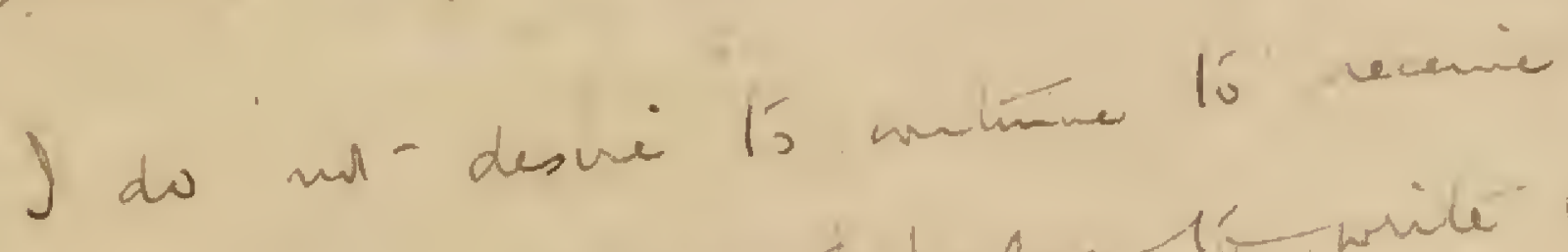

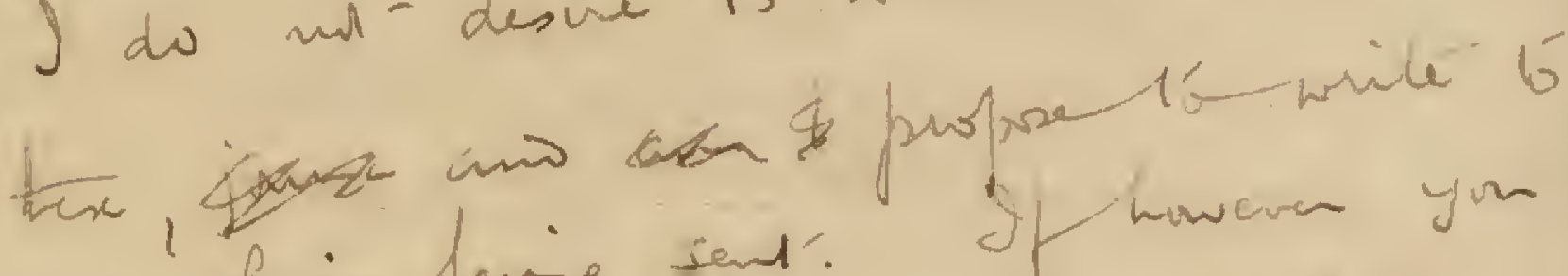
slipp their tevip sent. If noweren you do war abeas receme any of Leñ Inill repuest liat - They shased be

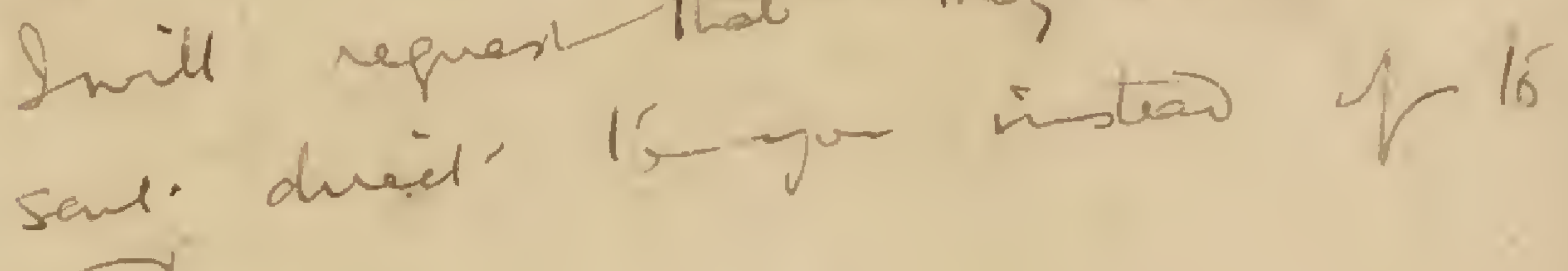
Jiparen.

E1.45 32 
3 in $422 / 29$.

3 rd september. 29.

Sir,

I have the honour to acknowledge the receipt of your letter of August 27 th in regard to certain periodicals of ered to you from the Gardens Libraryy anci note that you would glad to receive them, with certain exceptions

I poppose however to send you complete series of the India Rubber Journal and the India Rubier World, as odd volumes will be quite useless here.

I will advise you later when the volumes will be ready for despatch.

I have the honour to be,

Sir,

Your odedientsservant,

Director of Gardens, S.S.

The Editor,

Malayan Agricultural Journal, Department of Agaieulture, Kuala Lumpur. 
G 17

DRAFT

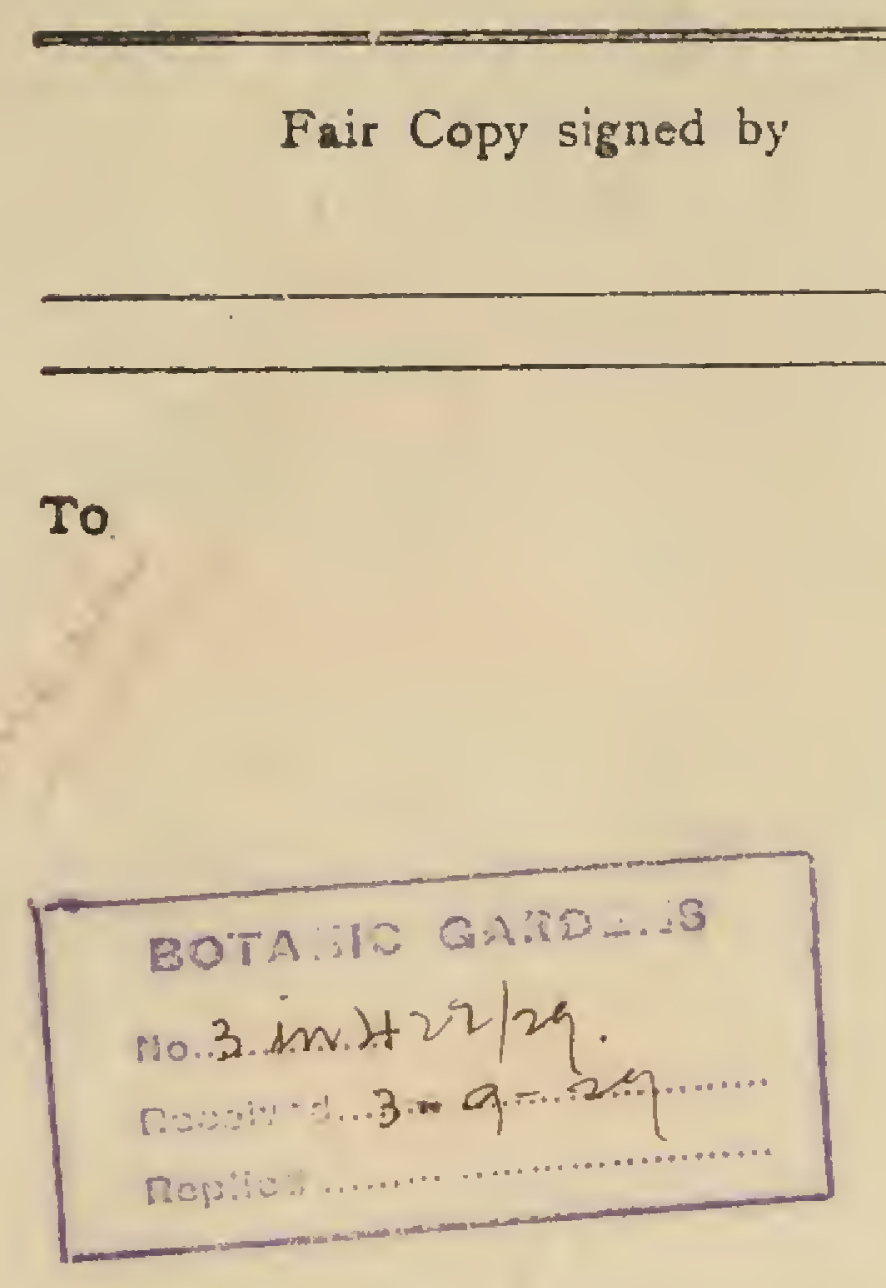

The Livin ma.t

B1.45134
$3-5-29$

Sike,

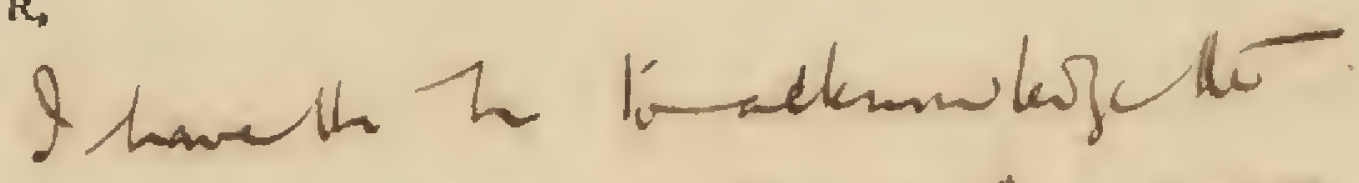

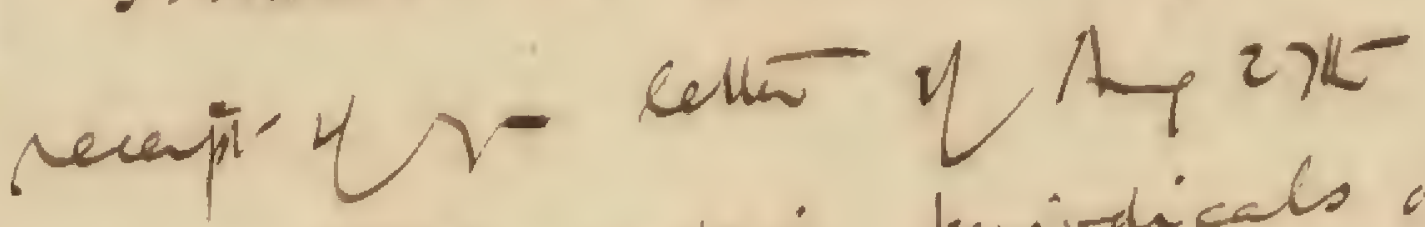
wregar tó cerlini paridicals offered ryan fordte fastem Litran, $r$ mole tad zu wanki he glas to ecene them, rit cavan exceptinas. 2. Q parpare limeres if ando youmbleler senies "L lle

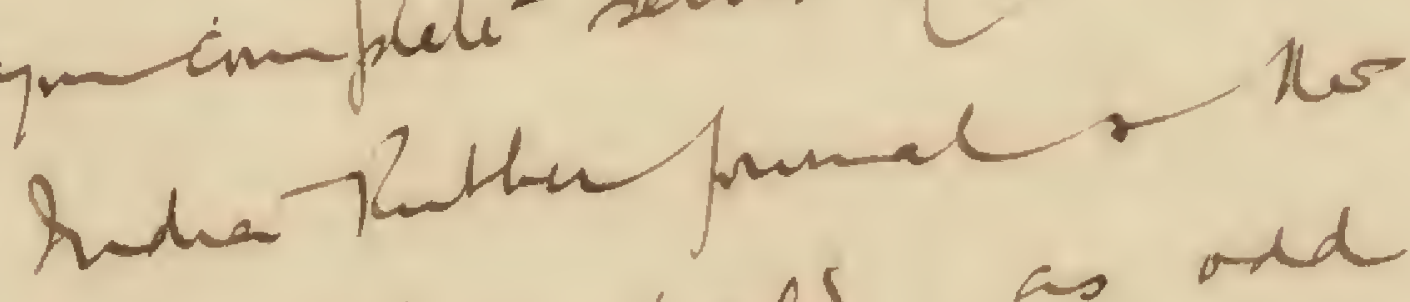

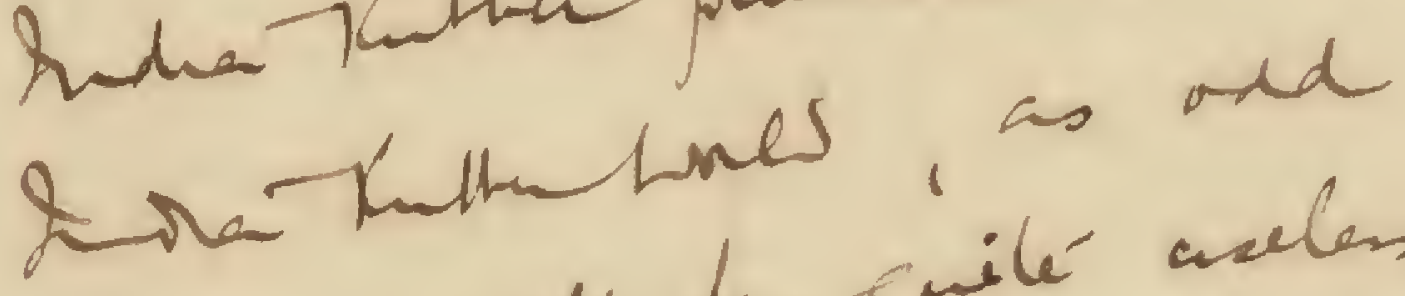

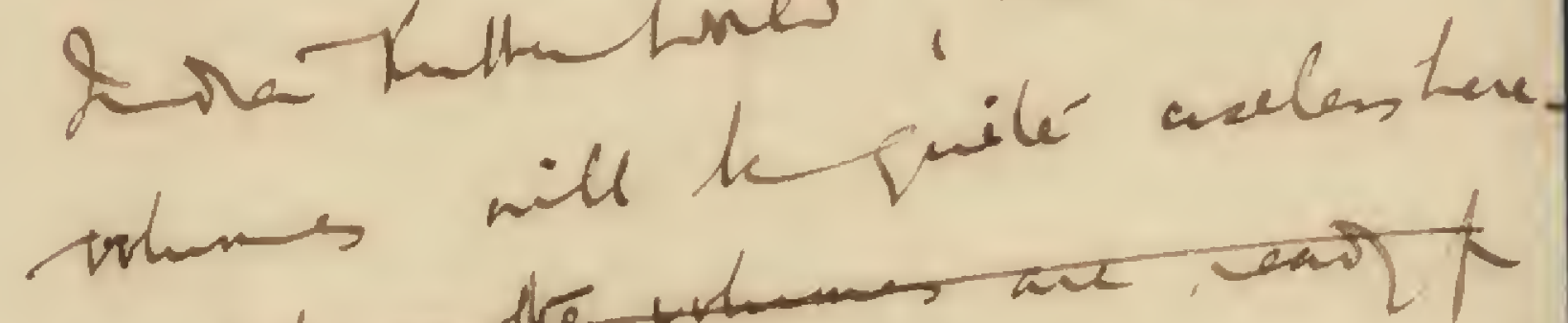

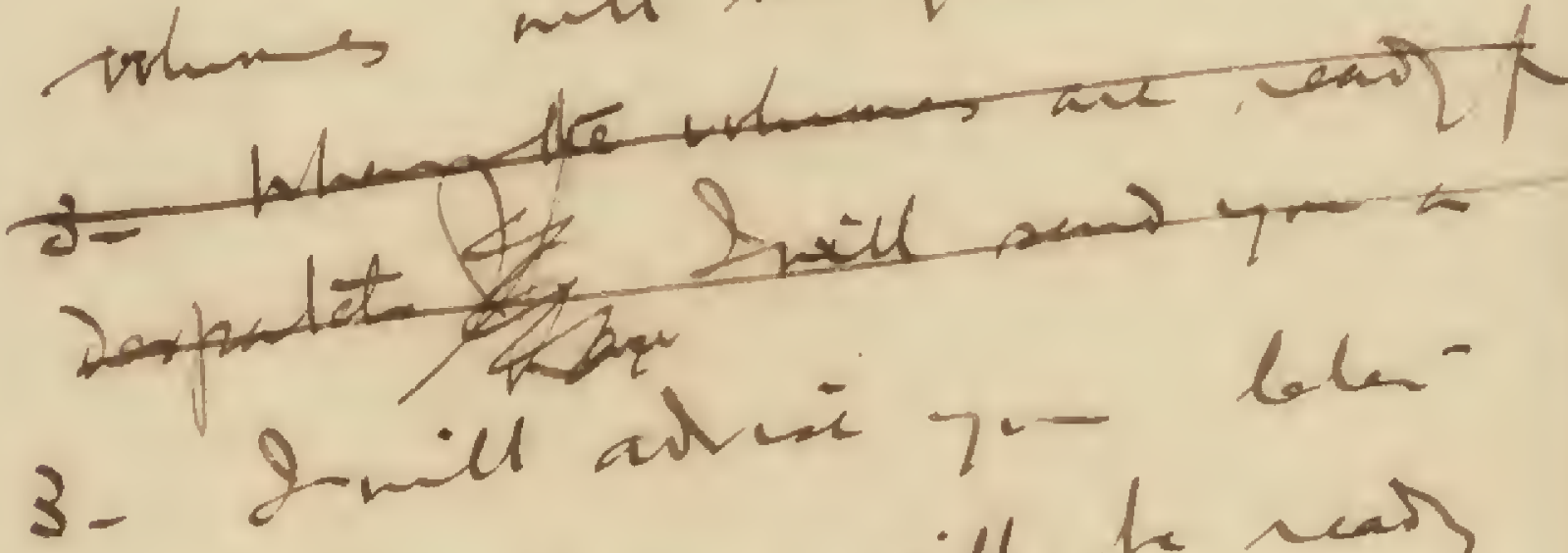

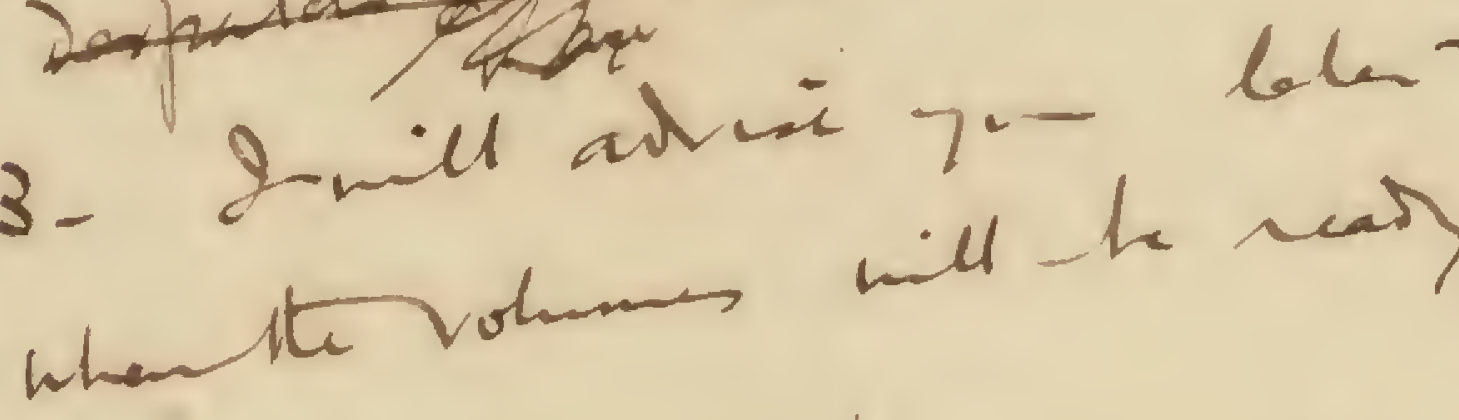

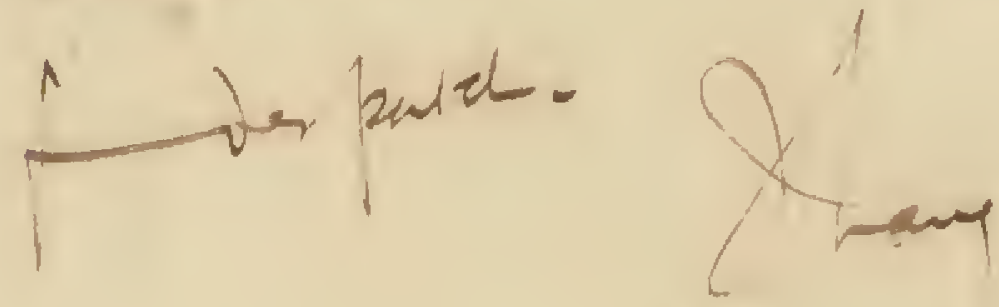

SE 
It 1 Hequested that the following number be quoted in the rept? to this letter.

$\mathscr{N}$
DEPARTMENT OF AGRICULTURE.

STRAITS SETTLEMENTS

\&

FEDERATED MALAy States.

Kuala Iumpur, 27 th August, 1929.
EOTAFIO GARDE
Mo.kin HU/2q

Sir,

I have the honour to acknowledge, with thanks, the receipt of your letter No.4Ez/29 dated zand August, 1929 and to inform you that I shall be glad to receive the periodicals for our Iibrary with the exception of the undermentioned periodicals of which only the numbers stated against each are required. Agricultural Gazette of New South Wales 1907-1909\& 1913. Agricultural Jouxnal of South Africa 1913 (VoI:VI) \& 1914. India Rubber Journal 1904 \& 1909-1928. India Rubber World Vols: $25,38,4 z, 43,45,46$, $48-50,52-54,58-60,63-68$ and 72 .

I have the honour to be, Sir,

Your obedient servant,

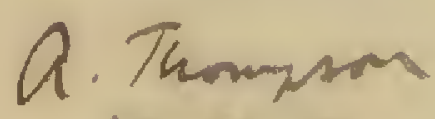

EDITOR,

Malayan Agricultural. Journal.

The Director of Gardens, S.S. Singapore.

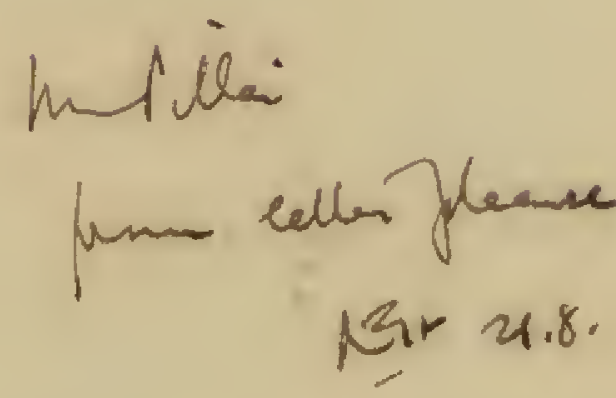

Su

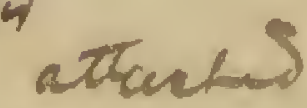


Sir,

I have the honour to enquire whether you would

like to take over for the library of your Department the undermentioned periodicals, which are not required in the Gardens Library. Journals marked. are still being received as exchanges for the Gardens Bulletin. I propose to stop these exchanges.

WAnnales des Planteurs de Caoutchouc de $L^{\prime}$ Indochine 1911-1918. Halletin de la Chambre d 'Agriculturede la Cochinchine 1908-1920 $\checkmark \times \vee B u l l e t i n$ de la chambre d'Agriculture du Tonkin 1910 -.-

4 Bulletin du Syndicat des Planteurs de Caoutchouc (Saigon) 1918-Whe Rhodesia Agricultural Journal 1909-.. 19t6. Journal of the Department of Agriculture West Australia1900-1908 Githe Agricultural Gazet.t New South Wales 1907--19i4.

" Mgricultural Journal of South Africa 1911--1914./ VII Mgricultural Journal of Victoria $1904--1920.4 \frac{\pi}{1}-\mathrm{XVII}$ Watal Agricultural Journal $1904-1910$.

Transvaal Agricultural Journal 1909.

$\int$ LGenier's Rubber News vol I - XI.

IrIndia Rubber Journal 1904 - 1928.

(') India Rubber Worla $1901--1925$.

VTopical Life. (odds)

¿La at acienda volo

$$
\text { 1) Ledelo. Your obedient servant, }
$$

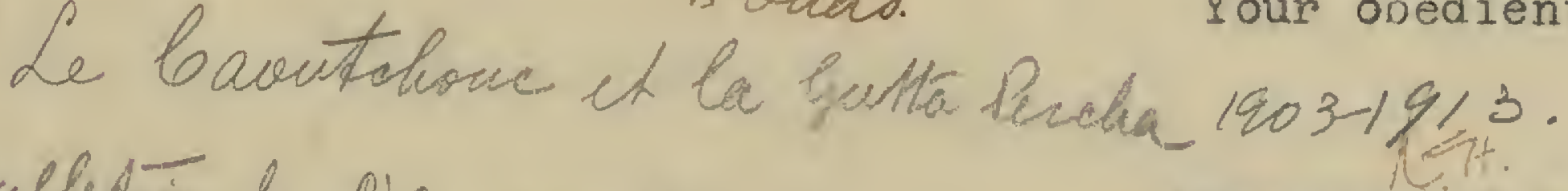

I have the honour to be,

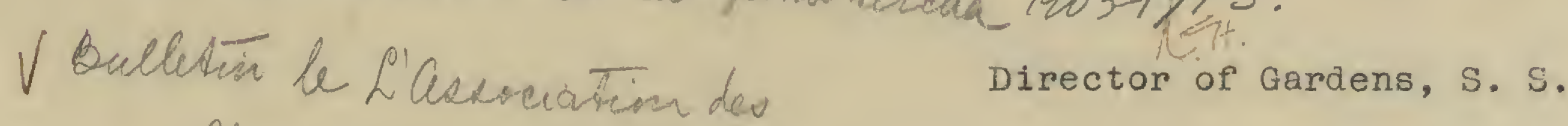

Pauteurs de Caoutes

Editor,

Agriculdumb

Malayan Agricultural Journal, Department of Agriculture, Kuala Lumpur, F. M. S. 
G 17

DRAFT

Fair Copy signed by

$22-8-29$

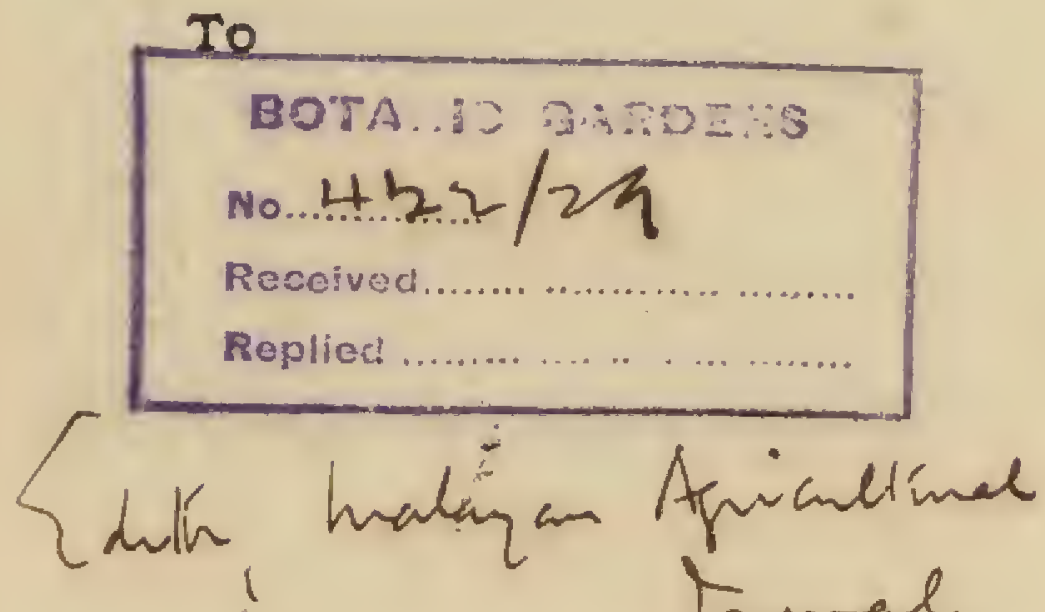

Leph I Apriculine

us.
Sik,

thanethe In t's enqune whethen Ut toe you nues like lo taka war fo fuar itinan 1 yar Departmant the unsermentimans perivodicals, which are

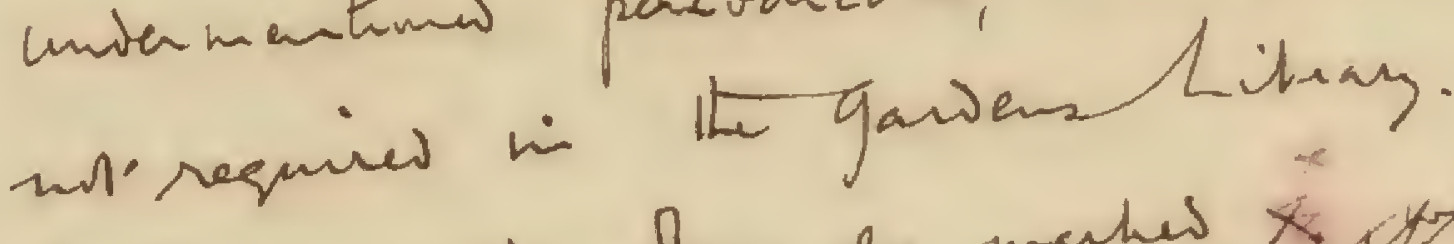

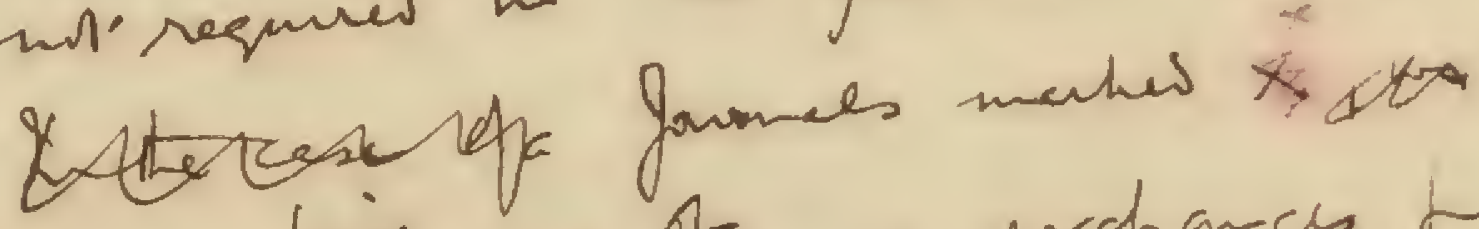

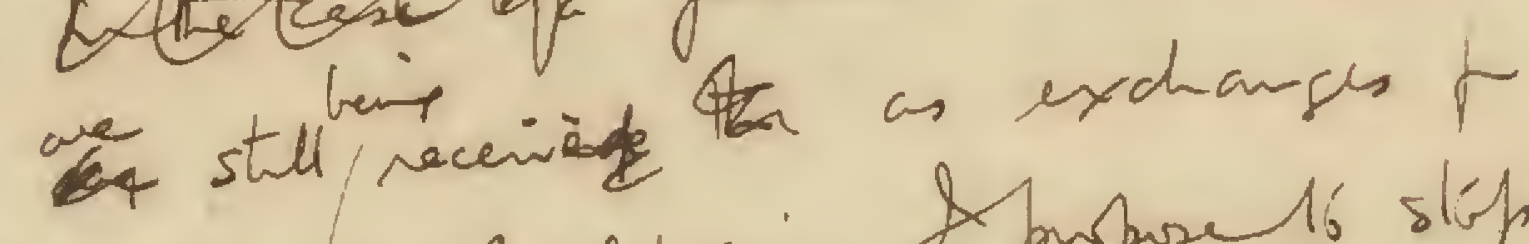

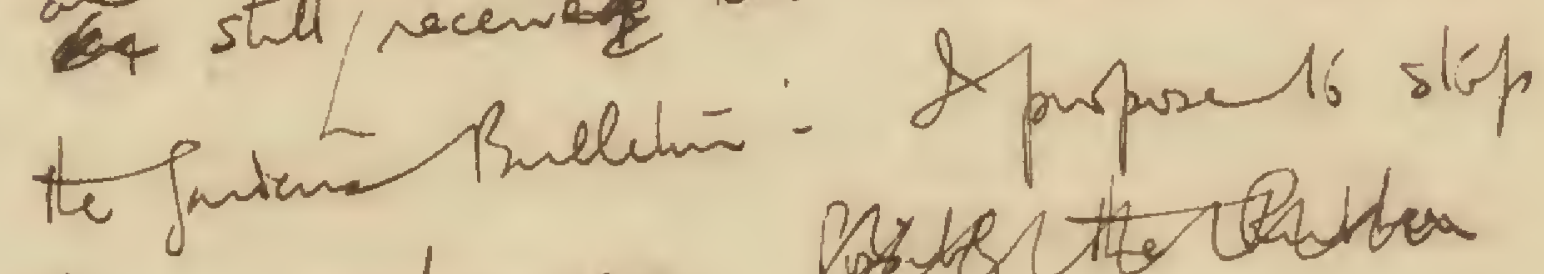

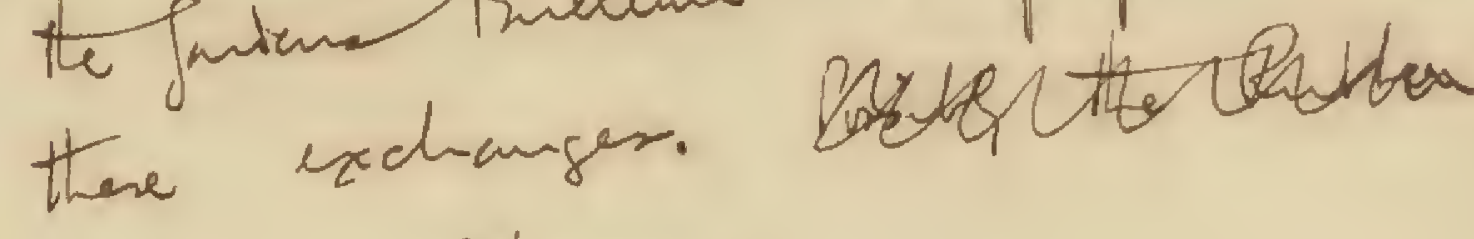
than

kin

Natal Atricielinal fumal 1904-1910

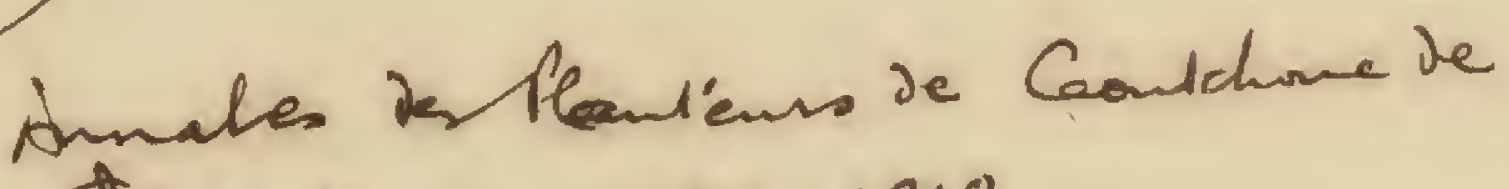
1. \$2 sosochini $1911-1918$

Bulletin relachanhe ritprianlinute la Cochnidmi 1909-1920

Bulletin dela chambe SAgrimelime on Touncin

1912

Rulletin on Symicicd. Des Plantems de Cavalichmo (Saign) 1918-

the Rhodesic Africultivel founal 1909 -

B1. 05130 
Thi Aguicultiral Gasetes NSW. $1907-1914$ Hith of the Depf Ager W. Amingoo - 1908

Agl. Il. 5 Afuce $1911-1914$

Agl gl. If cetrin 1904 - 1920.

Ag of of Cape Colomy $1888-1910$ Traonsaal. Agl fol 1909. H

Gremins Rublen Nuos val1-XI

Andea Rubler Goun. XXYI. (19 ou 1928$)$ Luo Rub Wonto tot2s (19m $1925 \%)$

inopual Life vat I (19os) $\rightarrow$ )

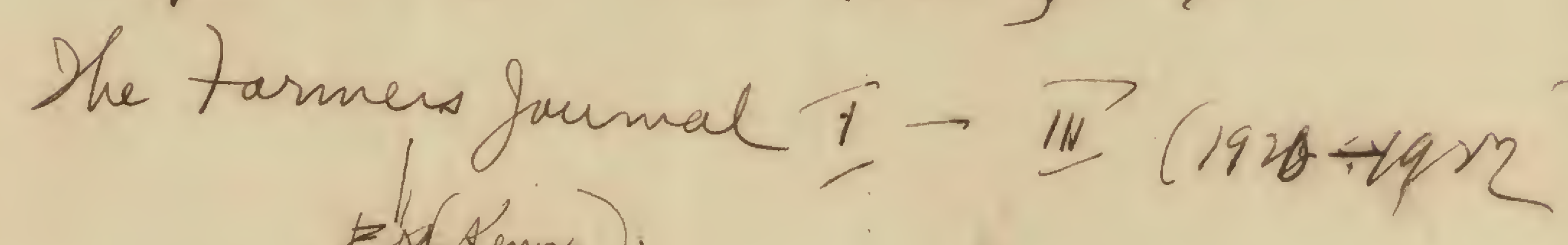

E. Alemp. .

B1.4538 